\title{
Compatible braidings with Hopf links, multiloop, and Borromean rings in (3+1)-dimensional spacetime
}

\author{
Zhi-Feng Zhang and Peng Ye* \\ School of Physics and State Key Laboratory of Optoelectronic Materials and Technologies, Sun Yat-sen University, Guangzhou, 510275, China
}

(Received 3 February 2021; accepted 28 April 2021; published 20 May 2021)

\begin{abstract}
Braiding phases among topological excitations are key data for physically characterizing topological orders. In this paper, we provide a field-theoretical approach toward a complete list of mutually compatible braiding phases of topological orders in $(3+1) \mathrm{D}$ spacetime. More concretely, considering a discrete gauge group as input data, topological excitations in this paper are bosonic particles carrying gauge charges and loops carrying gauge fluxes. Among these excitations, there are three classes of root braiding processes: particle-loop braidings (i.e., the familiar Aharonov-Bohm phase of winding an electric charge around a thin magnetic solenoid), multiloop braidings [Wang and Levin, Phys. Rev. Lett. 113, 080403 (2014)], and particle-loop-loop braidings (i.e., Borromean rings braiding in Chan et al. [Phys. Rev. Lett. 121, 061601 (2018)]). A naive way to exhaust all topological orders is to arbitrarily combine these root braiding processes. Surprisingly, we find that there exist illegitimate combinations in which certain braiding phases cannot coexist, i.e., are mutually incompatible. Thus, the resulting topological orders are illegitimate and must be excluded. It is not obvious to identify these illegitimate combinations. But with the help of the powerful (3+1)D topological quantum field theories (TQFTs), we find that illegitimate combinations violate gauge invariance. In this way, we are able to obtain all sets of mutually compatible braiding phases and all legitimate topological orders. To illustrate, we work out all details when gauge groups are $\mathbb{Z}_{N_{1}}, \mathbb{Z}_{N_{1}} \times \mathbb{Z}_{N_{2}}, \mathbb{Z}_{N_{1}} \times \mathbb{Z}_{N_{2}} \times \mathbb{Z}_{N_{3}}$ and $\mathbb{Z}_{N_{1}} \times \mathbb{Z}_{N_{2}} \times \mathbb{Z}_{N_{3}} \times \mathbb{Z}_{N_{4}}$. Finally, we concisely discuss compatible braidings and TQFTs in (4+1)D spacetime.
\end{abstract}

DOI: 10.1103/PhysRevResearch.3.023132

\section{INTRODUCTION}

The order parameter, which is designed for characterizing orders, is one of fundamental concepts of many-body physics. Symmetry-breaking orders are characterized by local order parameters-local functions of spacetime. However, topological orders (e.g., fractional quantum Hall states) [1-4] in gapped systems are characterized by intrinsically nonlocal order parameters, such as adiabatic quantum phases accumulated by braiding topological excitations (e.g., anyons) [5]. In topological orders, topological excitations are usually geometrically compact manifoldlike after taking continuum limits, such as pointlike particle excitations, stringlike loop excitations, etc. ${ }^{1}$ Braiding phases of topological excitations are proportional to integer-valued invariants of knots or links formed by world lines of particles and world sheets of loops, thereby being quantized and robust against local perturbations. In addition to topological orders, braiding phases have also been applied to characterization of symmetryprotected topological (SPT) phases [7-11] despite that SPT bulk excitations are topologically trivial. The core reason is

\footnotetext{
*yepeng5@mail.sysu.edu.cn

${ }^{1}$ For nonmanifoldlike excitations, Ref. [6] provides some examples in a class of exotic stabilizer codes that support spatially extended excitations with restricted mobility and deformability.

Published by the American Physical Society under the terms of the Creative Commons Attribution 4.0 International license. Further distribution of this work must maintain attribution to the author(s) and the published article's title, journal citation, and DOI.
}

that SPTs can be properly dualized to specific topological orders [7].

One quantitatively efficient and powerful approach to braiding phases is topological quantum field theory (TQFT) [12]. For example, braiding data of two-dimensional topological orders are encoded in $(2+1) \mathrm{D}$ Chern-Simons theory $[2,5,13]$. In this paper, we focus on deconfined phases of $(3+1) \mathrm{D}$ discrete gauge theories ${ }^{2}$ [14-18] with Abelian gauge group $G=\prod_{i=1}^{n} \mathbb{Z}_{N_{i}}$, where $n$ denotes the total number of cyclic subgroups. Such phases of matter are usually called $\prod_{i}^{n} \mathbb{Z}_{N_{i}}$ topological order. For example, the ground state of the three-dimensional toric code model $[19,20]$ admits $\mathbb{Z}_{2}$ topological order. By using group representation and a conjugacy class [21], we may label topological excitations via gauge charges and gauge fluxes. More specifically, there are totally $\prod_{i=1}^{n} N_{i}$ distinct bosonic particles carrying gauge charges and $\prod_{i=1}^{n} N_{i}$ distinct loops carrying gauge fluxes. Without loss of generality, it is enough to consider braiding phases among $n$ distinct elementary particles (denoted as $\left.e_{1}, e_{2}, \cdots, e_{i}, \cdots, e_{n}\right)$ carrying the unit gauge charge of a specific gauge subgroup and $n$ distinct elementary loops (denoted as $m_{1}, m_{2}, \cdots, m_{i}, \cdots, m_{n}$ ) carrying the unit gauge

\footnotetext{
${ }^{2}$ To reconcile different conventions in condensed-matter physics and high-energy physics, we take the following convention: gauge theories and field theories are always associated with spacetime dimensions but quantum states, Hamiltonian-type lattice models, or topological phases of matter (topological order, SPTs, etc.) are associated with spatial dimensions only.
} 
TABLE I. Compatible braiding phases and TQFTs when $G=\mathbb{Z}_{N_{1}}$ and $G=\mathbb{Z}_{N_{1}} \times \mathbb{Z}_{N_{2}}$. Each row represents a class of topological order(s). Thefirst row stands for a topological order whose braiding data is given by $\left\{\Theta_{i}^{\mathrm{H}}\right\}_{\mathbb{Z}_{N_{1}}}$ with $i=1,2,3$. The second row represents topological orders which are characterized by $\left\{\Theta_{i}^{\mathrm{H}}\right.$, any combinations of compatible $\Theta^{3 \mathrm{~L}}$ 's $\}$. The coefficient of $A A d A$ term is given by $q_{i j j}=\frac{n N_{i} N_{j}}{N_{i j}}, n \in$ $\mathbb{Z}_{N_{i j}}$, which is determined by the large gauge invariance. $\chi$ and $V$ are zero-form and one-form compact $\mathbb{U}(1)$ gauge parameters with $\int d \chi \in$ $2 \pi \mathbb{Z}$ and $\int d V \in 2 \pi \mathbb{Z}$.

\begin{tabular}{lcc}
\hline \hline Compatible braiding phases & TQFT actions & Gauge transformations \\
\hline$\Theta_{1}^{\mathrm{H}}=\frac{2 \pi}{N_{1}} \mathbb{Z}_{N_{1}}$ & $\int \frac{N_{1}}{2 \pi} B^{1} d A^{1}$ & $A^{1} \rightarrow A^{1}+d \chi^{1}$ \\
& $B^{1} \rightarrow B^{1}+d V^{1}$ & $A^{i} \rightarrow d \chi^{i}$ \\
$\Theta_{i}^{\mathrm{H}}=\frac{2 \pi}{N_{i}}$ & $\int \sum_{i=1}^{2} \frac{N_{i}}{2 \pi} B^{i} d A^{i}+\frac{q_{122}}{(2 \pi)^{2}} A^{1} A^{2} d A^{2}$ & $B^{1} \rightarrow B^{1}+d V^{1}$ \\
$\Theta_{22 \mid 1}^{3 \mathrm{~L}}=-2 \cdot \Theta_{12 \mid 2}^{3 \mathrm{~L}}=\frac{4 \pi q_{122}}{N_{1} N_{2}}$ & $+\frac{q_{211}}{(2 \pi)^{2}} A^{2} A^{1} d A^{1}$ & $+\frac{q_{122}}{(2 \pi) N_{1}} d \chi^{2} A^{2}-\frac{q_{211}}{(2 \pi) N_{1}} d \chi^{2} A^{1}$ \\
$\Theta_{11 \mid 2}^{3 \mathrm{~L}}=-2 \cdot \Theta_{21 \mid 1}^{3 \mathrm{~L}}=\frac{4 \pi q_{211}}{N_{1} N_{2}}$ & & $B^{2}+d V^{2}$ \\
& & $-\frac{q_{122}}{(2 \pi) N_{2}} d \chi^{1} A^{2}+\frac{q_{211}}{(2 \pi) N_{2}} d \chi^{1} A^{1}$ \\
\hline \hline
\end{tabular}

flux of a specific gauge subgroup. When more than one particle (loop) are simultaneously involved in the same braiding process, superscripts will be added properly to $e_{i}\left(m_{i}\right)$.

Among these elementary excitations, there exist three classes of braiding processes that have been studied before: particle-loop braiding [15,16,22-25], multiloop braiding [26-44], and particle-loop-loop braiding [i.e., Borromean rings (BR) braiding] [45]. For the purposes of this paper, we regard these braidings as root braiding processes. Within each class of root braiding processes, depending on gauge group assignment, there are still many different braiding phases among which compatibility is crucial. To proceed further, let us briefly introduce the three classes of root braidings.

In the first class, within each gauge subgroup, e.g., $\mathbb{Z}_{N_{i}}$, there is a well-defined particle-loop braiding phase $\Theta_{i}^{\mathrm{H}}=$ $\frac{2 \pi}{N_{i}} \bmod 2 \pi$ when the Hopf linking invariant is one. ${ }^{3}$ Here, the Hopf link is formed by an elementary particle's trajectory $\gamma_{e_{i}}$ and an elementary loop $m_{i}$. This braiding phase always exists since it physically encodes the cyclic group structure of $\mathbb{Z}_{N_{i}}$. For the whole gauge group, the root braiding phases of the first class form a set $\left\{\Theta_{i}^{\mathrm{H}}\right\}_{G}$ with $i=1, \cdots, n$. A subscript $G$ is added for specifying gauge group $G$. Apparently, all braiding phases in the set belong to distinct gauge subgroups, thereby being mutually compatible and linearly independent. In the language of TQFT, one may compute braiding phases from gauge-invariant correlation functions of Wilson operators of the topological $B F$ theories with action $S_{B F}=\int \sum_{i} \frac{N_{i}}{2 \pi} B^{i} \wedge$ $d A^{i}$ (abbreviated as $B d A$ ) [16,46-49]. Here, the one-form $A^{i}$ and two-form $B^{i}$ are compact $\mathbb{U}(1)$ gauge fields describing the loop current $\left(\frac{1}{2 \pi} * d A^{i}\right)$ and particle current $\left(\frac{1}{2 \pi} * d B^{i}\right)$ degrees of freedom, respectively. As a natural higher-dimensional generalization of the Chern-Simons theory, the $B F$ theory has been broadly applied to condensed matter systems, such as superconductors [16], bosonic and fractional topological insulators, and more general 3D SPTs [49-53].

\footnotetext{
${ }^{3}$ The superscript $H$ in $\Theta_{i}^{\mathrm{H}}$ stands for Hopf. As an angle, the $2 \pi$ period is important but obvious, so we will not write it explicitly hereafter. To characterize topological orders, it is sufficient to consider braiding processes in which the linking number is unit.
}

In the second class, i.e., multiloop (three or four) braidings, all objects involved in the braidings are loops. More specifically, a three-loop braiding [26] consists of three elementary loops and lead to a set of braiding phases $\left\{\Theta_{i}^{\mathrm{H}} ; \Theta_{j, k \mid l}^{3 \mathrm{~L}}\right\}_{G}$, where $G=\prod_{i=1}^{n} \mathbb{Z}_{N_{i}}$ with $n \geqslant 2$. As mentioned above, when $G$ is given, $\Theta_{i}^{\mathrm{H}}$ always exists. Here $j, k, l$ indicate that three elementary loops (denoted by $m_{j}^{1}, m_{k}^{2}, m_{l}^{b}$ ), respectively, carry the elementary gauge flux of $\mathbb{Z}_{N_{j}}, \mathbb{Z}_{N_{k}}, \mathbb{Z}_{N_{l}}$ gauge subgroups. Geometrically, the loop $m_{l}^{b}$ right after the symbol |, which carries the elementary gauge flux of the $\mathbb{Z}_{N_{l}}$ gauge subgroup, is called base loop [26]. The latter is simultaneously hopfly linked to the other two loops, i.e., $m_{j}^{1}, m_{k}^{2}$. Under this geometric setting, the three-loop braiding can be regarded as an anyonic braiding process on the Seifert surface bounded by the base loop. Braiding phases in the set $\left\{\Theta_{i}^{\mathrm{H}} ; \Theta_{j, k \mid l}^{3 \mathrm{~L}}\right\}_{G}$ satisfy a series of remarkably elegant constraints such that mutually compatible braiding data sets can be unambiguously determined. The result was obtained in Ref. [26] by means of general properties of the discrete gauge group and adiabaticity of braiding processes. The same result can also be obtained from TQFTs with topological terms of $B d A+A A d A$ form [27-34,44]. Moreover, in the second class, if we consider four loops from four distinct gauge subgroups, the four-loop braiding phases form a set $\left\{\Theta_{i}^{\mathrm{H}} ; \Theta_{j, k, l, m}^{4 \mathrm{~L}}\right\}_{G}$, where $G=\prod_{i=1}^{n} \mathbb{Z}_{N_{i}}$, with $n \geqslant 4$, and the four loops carry elementary gauge fluxes of four different gauge subgroups $\mathbb{Z}_{N_{j}}, \mathbb{Z}_{N_{k}}, \mathbb{Z}_{N_{l}}, \mathbb{Z}_{N_{m}}$ respectively. The fourloop braiding is associated with the quadruple linking number of surfaces, thereby being quantized [28]. The corresponding TQFTs can be symbolically expressed as $B d A+A A A A$ [27-34,44].

In the third class, i.e., the particle-loop-loop braiding or BR braiding [45], an elementary particle carrying unit gauge charge of $\mathbb{Z}_{N_{k}}$ gauge subgroup moves around two loops (denoted by $m_{i}^{1}, m_{j}^{2}$ ) that, respectively, carry unit gauge fluxes of $\mathbb{Z}_{N_{i}}$ and $\mathbb{Z}_{N_{j}}$, such that the particle's trajectory $\gamma_{e_{k}}$ and the two loops together form aBR link, or general Brunnian link. The corresponding braiding phase is denoted as $\Theta_{i, j \mid k}^{\mathrm{BR}}$, which is proportional to the Milnor's triple linking number $\bar{\mu}$ [45,54,55]. Likewise, one may define a set of braiding phases: $\left\{\Theta_{i}^{\mathrm{H}} ; \Theta_{j, k \mid l}^{\mathrm{BR}}\right\}_{G}$, where $G=\prod_{i=1}^{n} \mathbb{Z}_{N_{i}}$ with $n \geqslant 3$. The corresponding 
TABLE II. Compatible braiding phases and TQFTs when $G=\mathbb{Z}_{N_{1}} \times \mathbb{Z}_{N_{2}} \times \mathbb{Z}_{N_{3}}$. Each row represents a class of topological orders which are characterized by braiding data $\left\{\Theta_{i}^{\mathrm{H}}\right.$; combinations of compatible braiding phases $\}$. Take the second row as an example: the sets of braiding phases $\left\{\Theta_{i}^{\mathrm{H}} ; \Theta_{3,3 \mid 2}^{3 \mathrm{~L}}\right\},\left\{\Theta_{i}^{\mathrm{H}} ; \Theta_{2,3 \mid 1}^{\mathrm{BR}}\right\},\left\{\Theta_{i}^{\mathrm{H}} ; \Theta_{3,3 \mid 2}^{3 \mathrm{~L}} ; \Theta_{2,3 \mid 1}^{\mathrm{BR}}\right\},\left\{\Theta_{i}^{\mathrm{H}} ; \Theta_{3,3 \mid 2}^{3 \mathrm{~L}}, \Theta_{2,3 \mid 3}^{3 \mathrm{~L}}, \Theta_{2,2 \mid 3}^{3 \mathrm{~L}}, \Theta_{3,2 \mid 2}^{3 \mathrm{~L}} ; \Theta_{2,3 \mid 1}^{\mathrm{BR}}\right\}$, etc., respectively, characterize a topological order. The coefficients are given by $q_{i j k}=\frac{m N_{i} N_{j}}{N_{i j}}, m \in \mathbb{Z}_{N_{i j k}}$, and $p_{i j, k}=\frac{l_{i j, k} N_{i} N_{j} N_{k}}{N_{i j k}}, l_{i j, k} \in \mathbb{Z}_{N_{i j k}}$, as a result of large gauge invariance. $l_{i j, k}=-l_{j i, k}$ and $l_{i j, k}=0$ if any of the two indices are the same. The Levi-Civita symbol is defined by $\epsilon^{a_{1} a_{2} \cdots a_{n}}=\prod_{1 \leqslant i<j \leqslant n} \operatorname{sgn}\left(a_{j}-a_{i}\right)$ where $\operatorname{sgn}(x)=1$, 0 , or -1 if $x>0, x=0$, or $x<0$.

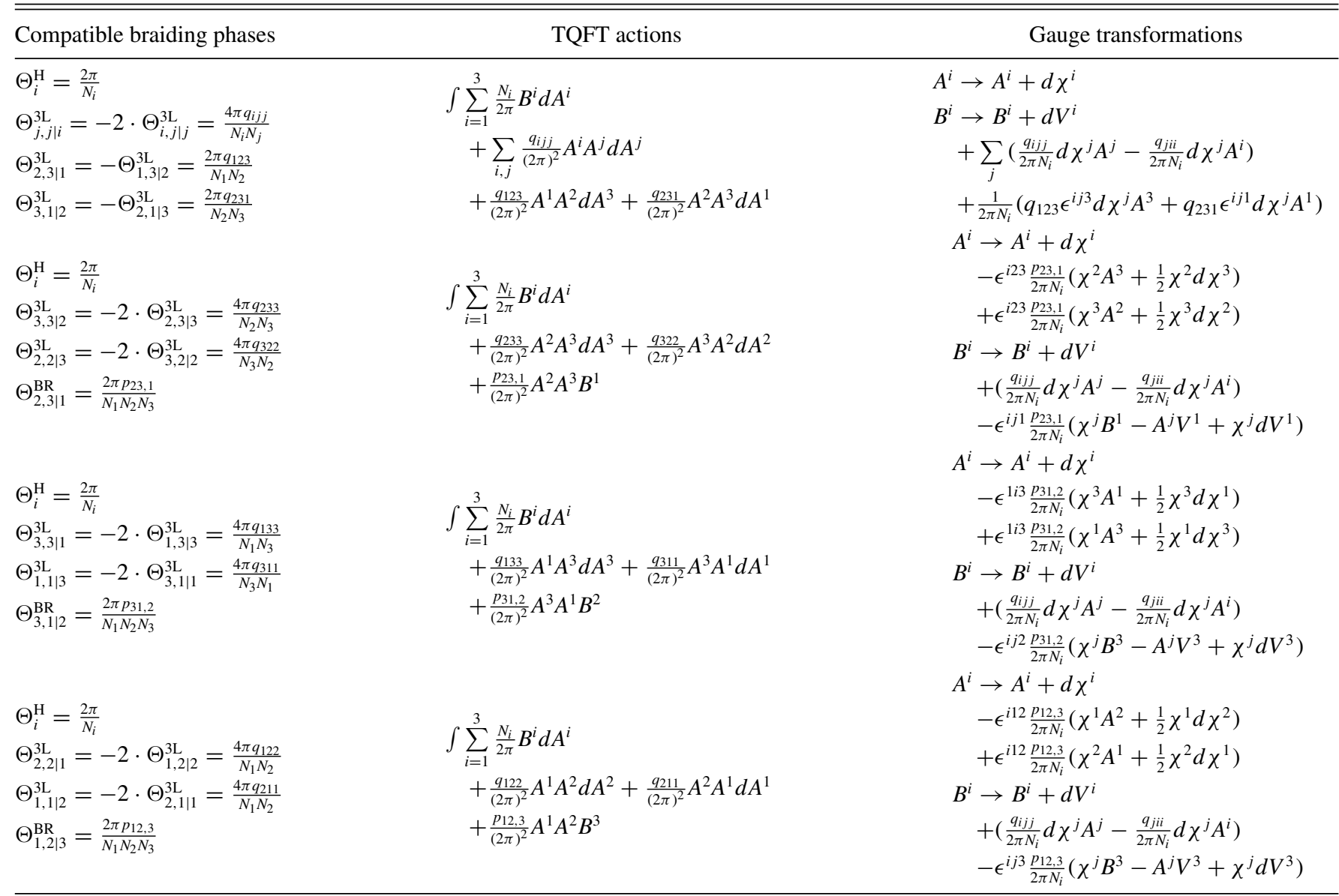

TQFTs can be symbolically expressed as $B d A+A A B$ [45].

In this paper, we put all root braiding processes, which are denoted by $\left\{\Theta_{i}^{\mathrm{H}} ; \Theta_{j, k \mid l}^{3 \mathrm{~L}} ; \Theta_{m, n, o, p}^{4 \mathrm{~L}} ; \Theta_{q, r \mid s}^{\mathrm{BR}}\right\}_{G}$, in arbitrary combinations and try to exhaust all topological orders. We find that not all possible gauge group assignments (i.e., the subscripts $i, j, k, l, \cdots)$ are realizable. Not all braiding phases in a given set are linearly independent. As we reviewed above, within each class, compatible braiding phases have been studied via various approaches. By compatible, we mean that these braiding processes can be supported in the same system. In other words, the compatible braiding phases together as a set of braidings characterize a legitimate topological order. If there are two mutually incompatible braiding processes in the set, then both braiding processes must always lead to two trivial braiding phases, i.e., $0 \bmod 2 \pi$ regardless of the values of linking numbers of the braidings. As we will show, gauge invariance is broken if any one of two braidings has a nontrivial braiding phase. Therefore, to exhaust all legitimate topological orders, it is sufficient to find all sets of braidings formed by mutually compatible braiding processes. For this purpose, in this paper, through TQFT approach, we compute all braiding processes in a unified framework and figure out all cases of incompatibility that are tightly related to gauge noninvariance. Especially, we focus on cases of incompatibility that occur when two or three distinct classes of root braiding processes have nontrivial braiding phases. Compatible braiding phases for different gauge groups are summarized in Table I $\left(G=\mathbb{Z}_{N}\right.$ and $\left(G=\mathbb{Z}_{N_{1}} \times \mathbb{Z}_{N_{2}}\right)$, Table II $\left(G=\mathbb{Z}_{N_{1}} \times \mathbb{Z}_{N_{2}} \times \mathbb{Z}_{N_{3}}\right)$, and Table III $\left(G=\mathbb{Z}_{N_{1}} \times \mathbb{Z}_{N_{2}} \times \mathbb{Z}_{N_{3}} \times \mathbb{Z}_{N_{4}}\right)$. More general cases with more than four $\mathbb{Z}_{N}$ gauge subgroups can be straightforwardly analyzed by applying the results in these four tables, as shown in Sec. IIID. In addition to braiding phases, in these tables, we also provide the corresponding TQFTs and definitions of gauge transformations therein. All other sets of braiding phases are incompatible and not realizable. Some typical examples of incompatibility will be analyzed in detail in this paper.

The remainder of this paper is structured as follows. In Sec. II, we concretely analyze root braiding processes one by one to lay the foundation for the forthcoming discussions on compatibility. In Sec. III, by combining all root braiding 
TABLE III. Compatible braiding phases and TQFTs when $G=\mathbb{Z}_{N_{1}} \times \mathbb{Z}_{N_{2}} \times \mathbb{Z}_{N_{3}} \times \mathbb{Z}_{N_{4}}$. Each row represents a class of topological orders which are characterized by braiding data $\left\{\Theta_{i}^{\mathrm{H}}\right.$; combinations of compatible braiding phases\}. Only 3 of 11 (see the main text) legitimate TQFT actions are listed here. General expressions of legitimate TQFT actions and gauge transformations are given in Table IV. By properly assigning the indices in the general expressions, one can obtain all legitimate TQFT actions for $G=\prod_{i=1}^{4} \mathbb{Z}_{N_{i}}$. Coefficients are given by $q_{i j k}=\frac{m N_{i} N_{j}}{N_{i j}}$, $m \in \mathbb{Z}_{N_{i j k}} ; q_{i j k l}=\frac{n N_{i} N_{j} N_{k} N_{l}}{N_{i j k l}}, n \in \mathbb{Z}_{N_{i j k l}}$ and $p_{i j, k}=\frac{l_{i j, k} N_{i} N_{j} N_{k}}{N_{i j k}}, l_{i j, k} \in \mathbb{Z}_{N_{i j k}}$ as a result of large gauge invariance. $l_{i j, k}=-l_{j i, k}$ and $l_{i j, k}=0$ if any of the two indices are the same. The Levi-Civita symbol is defined by $\epsilon^{a_{1} a_{2} \cdots a_{n}}=\prod_{1 \leqslant i<j \leqslant n} \operatorname{sgn}\left(a_{j}-a_{i}\right)$ where $\operatorname{sgn}(x)=1,0$, or -1 if $x>0$, $x=0$, or $x<0$.

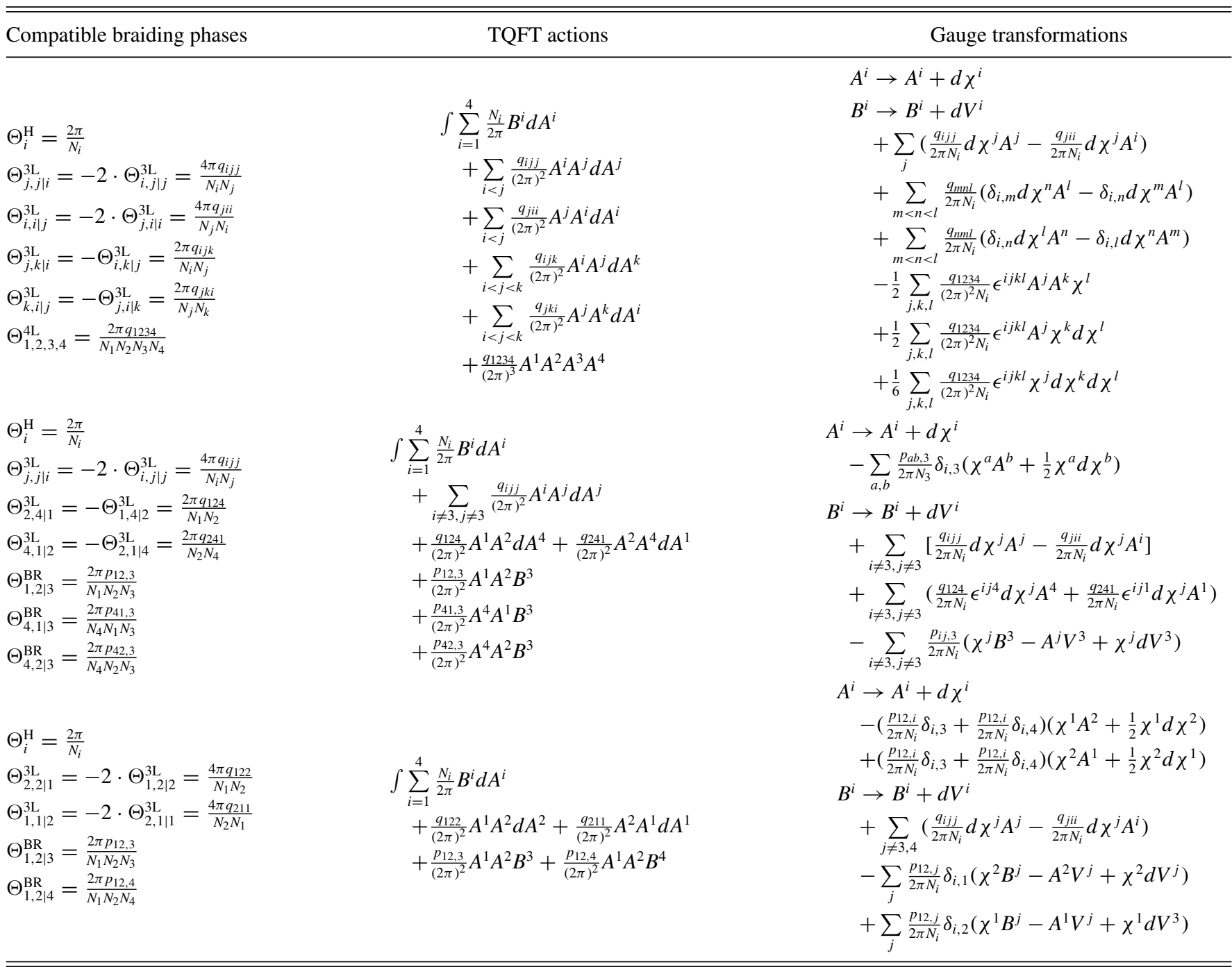

processes together, we study the corresponding TQFTs and extract all sets of compatible braiding processes. Then, in Sec. IV, some sets of incompatible braiding processes are illustrated in some concrete examples. In Sec. V, along the same line, we concisely discuss compatible braidings and TQFTs in $(4+1)$-dimensional topological orders. Conclusions are made in Sec. VI. Several technical details are collected in the Appendices.

\section{REVIEW ON ROOT BRAIDING PROCESSES AND GAUGE TRANSFORMATIONS}

In this section, we review TQFTs of root braiding processes. We emphasize the correspondence between root braiding processes, topological terms, and braiding phases, which is illustrated as the following triangle:

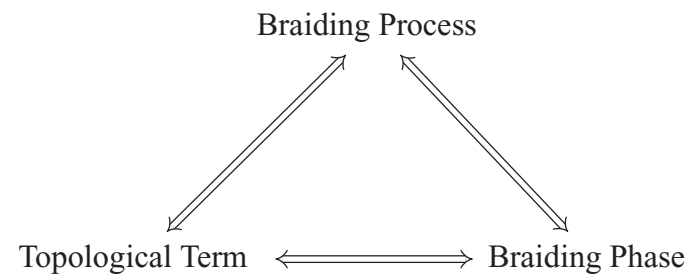

A braiding process can be identified from a topological term or a braiding phase, and vice versa. In this manner, we can study the braiding processes within the framework of TQFT. More concretely, to extract braiding phases from a TQFT, one can either add gauge-invariant source terms [30,45] or study algebra of Wilson operators $[34,40]$, such that the braiding phases of a given braiding process are connected to a 
linking or knot invariant formed by the spacetime trajectories of particles and loops.

\section{A. Microscopic origins of discrete gauge groups, topological excitations, and TQFT actions}

As our basic goal is to utilize TQFTs and braiding processes to characterize and classify topological orders of underlying quantum many-body systems, it is very important to identify microscopic origins of input data of TQFTs and braiding processes.

Hamiltonian realization, especially an exactly solvable model proposal, is always the most powerful stimulus of the progress of topological orders. For example, the toric code model proposed by Kitaev [20] elegantly unveils all key properties of nonchiral Abelian topological orders in $(2+1) \mathrm{D}$. String-net models constructed by Levin and Wen [56] are applied to exhaust non-Abelian topological orders in $(2+$ 1)D. In $(3+1) \mathrm{D}$, topological orders that are within DijkgraafWitten cohomology classification [57] have Hamiltonian realization in Ref. [38], which uses four-cocycles to cover all particle-loop and multiloop braidings. However, exactly solvable models for BR braiding [45] are not known, which is an interesting future direction. References $[58,59]$ present strategies for constructing higher-dimensional Abelian and non-Abelian topological phases via coupling quantum wires, which may shed light on constructing exactly solvable lattice model for BR braiding.

While exactly solvable models have Hamiltonian form, the Hamiltonians often look very intricate and unrealistic (four-spin, six-spin interactions, and more). Furthermore, it is unclear for us to rigorously connect the Hamiltonians to TQFTs. The latter have been proved to be a very powerful machine to study topological orders since the discovery of the fractional quantum Hall effect. But, to the best of our knowledge, it is highly impossible to perform a standard perturbation theory to renormalize the intricate interacting electron system of the $v=1 / 3$ Laughlin state to a beautiful Chern-Simons gauge theory $\int \frac{3}{4 \pi} A d A$, where $A$ is an emergent gauge field. Although there are many effective ways, e.g., parton construction and hydrodynamical approach, to handle strongly correlated physics, it is still kind of mysterious to derive emergent dynamical gauge fields from the very beginning.

To identify microscopic origins, below we will provide an effective way of thinking, following the spirit of previous works $[16,29,34,50,52,60]$. From this effective derivation, we can find how discrete gauge groups, topological terms, and quantized Wilson integrals arise from a quantum many-body system. We can also find that particle excitations and loop excitations are gauge charges and gauge fluxes of gauge groups. Below, we take $\int B d A+A A B$ as an example by means of exotic boson condensate and vortexline condensate. The detailed derivation is given in Appendix A. Here we just briefly sketch the key idea.

We start from a multilayer condensate in 3D space in which one layer is in the vortexline condensation phase (from disordering a 3D superfluid) while the others are in charge condensation phases (bosonic superconductors): The former can be regarded as the Higgs phase of two-form gauge fields while the latter can be regarded as the usual Higgs phase of one-form gauge fields,

$$
\begin{aligned}
\mathcal{L}= & \frac{\rho_{3}}{2}\left(\partial_{[\mu} \Theta_{\nu]}-N_{3} B_{\mu \nu}^{3}\right)^{2}+\frac{\rho_{1}}{2}\left(\partial_{\mu} \theta^{1}-N_{1} A_{\mu}^{1}\right)^{2} \\
& +\frac{\rho_{2}}{2}\left(\partial_{\mu} \theta^{2}-N_{2} A_{\mu}^{2}\right)^{2}+i \Lambda \varepsilon^{\mu \nu \lambda \rho}\left(\partial_{[\mu} \Theta_{\nu]}-N_{3} B_{\mu \nu}^{3}\right) \\
& \times\left(\partial_{\lambda} \theta^{1}-N_{1} A_{\lambda}^{1}\right)\left(\partial_{\rho} \theta^{2}-N_{2} A_{\rho}^{2}\right),
\end{aligned}
$$

where $\partial_{[\mu} \Theta_{\nu]}=\partial_{\mu} \Theta_{\nu}-\partial_{\nu} \Theta_{\mu}$. The vectorlike phase angle $\Theta_{\mu}$ describes the phase field of vortexline condensation [50], while $\theta^{1}$ and $\theta^{2}$ are the usual phase angles of boson condensation. The coefficients $\rho_{1}, \rho_{2}, \rho_{3}$ represent phase rigidity of condensates. The last term of the above Lagrangian couple three condensates together in a gauge-invariant fashion. By introducing Hubbard-Stratonovich fields $\Sigma_{\mu \nu}^{3}, j^{1}, j^{2}$ and Lagrange multiplier fields $\xi^{I}$ and $\eta^{I}$, we obtain

$$
\begin{aligned}
\mathcal{L}= & \frac{1}{2 \rho_{1}}\left(j^{1}\right)^{2}-i \theta^{1} \partial_{\lambda} j_{\lambda}^{1}-i N_{1} A_{\lambda}^{1} j_{\lambda}^{1}+\frac{1}{2 \rho_{2}}\left(j^{2}\right)^{2}-i \theta^{2} \partial_{\rho} j_{\rho}^{2}-i N_{2} A_{\rho}^{2} j_{\rho}^{2}+\frac{1}{8 \rho_{3}}\left(\Sigma_{\mu \nu}^{3}\right)^{2}-i \Theta_{\mu} \partial_{\nu} \Sigma_{\mu \nu}^{3}-i \frac{1}{2} N_{3} B_{\mu \nu}^{3} \Sigma_{\mu \nu}^{3} \\
& +i \Lambda \varepsilon^{\mu \nu \lambda \rho}\left[2 \Theta_{\mu} \partial_{\nu}\left(N_{1} N_{2} A_{\lambda}^{1} A_{\rho}^{2}\right)+\theta^{1} \partial_{\lambda}\left(N_{2} A_{\rho}^{2} N_{3} B_{\mu \nu}^{3}\right)+\theta^{2} \partial_{\rho}\left(N_{3} B_{\mu \nu}^{3} N_{1} A_{\lambda}^{1}\right)\right] \\
& +i \eta_{\lambda}^{1}\left[\xi_{\lambda}^{1}-\Lambda \varepsilon^{\mu \nu \lambda \rho} \cdot \frac{1}{2} \partial_{\rho} \theta^{2} N_{3} B_{\mu \nu}^{3}\right]+i \theta^{1} \partial_{\lambda} \xi_{\lambda}^{1}+i \eta_{\rho}^{2}\left[\xi_{\rho}^{2}-\Lambda \varepsilon^{\mu \nu \lambda \rho} \cdot \frac{1}{2} \partial_{\lambda} \theta^{1} N_{3} B_{\mu \nu}^{3}\right]+i \theta^{2} \partial_{\rho} \xi_{\rho}^{2} \\
& +i \eta_{\mu \nu}^{3}\left[\xi_{\mu \nu}^{3}-\Lambda \varepsilon^{\mu \nu \lambda \rho}\left(2 \partial_{\lambda} \theta^{1} N_{1} A_{\rho}^{2}+2 \partial_{\rho} \theta^{2} N_{1} A_{\lambda}^{1}\right)\right]+i \Theta_{\mu} \partial_{\nu} \xi_{\mu \nu}^{3}-i N_{1} N_{2} N_{3} \Lambda \varepsilon^{\mu \nu \lambda \rho} A_{\lambda}^{1} A_{\rho}^{2} B_{\mu \nu}^{3}+\text { boundary terms. }
\end{aligned}
$$

Integrating out $\Theta_{\mu}, \theta^{1}$ and $\theta^{2}$ yields constraints in the path-integral measure. These constraints can be solved by introducing one-form gauge field $A^{3}$, two-form gauge fields $B^{1}$ and $B^{2}$, respectively:

$$
\begin{aligned}
\Sigma_{\mu \nu}^{3} & =\frac{1}{2 \pi} \varepsilon^{\mu \nu \lambda \rho} \partial_{\lambda} A_{\rho}^{3}+\xi_{\mu \nu}^{3}-\Lambda \varepsilon^{\mu \nu \lambda \rho} \times 2 N_{1} N_{2} A_{\lambda}^{1} A_{\rho}^{2}, \\
j_{\lambda}^{1} & =\frac{1}{4 \pi} \varepsilon^{\lambda \rho \mu \nu} \partial_{\rho} B_{\mu \nu}^{1}+\xi_{\lambda}^{1}-\Lambda \varepsilon^{\mu \nu \lambda \rho}\left(N_{2} A_{\rho}^{2} N_{3} B_{\mu \nu}^{3}-\frac{1}{2} \eta_{\rho}^{2} N_{3} B_{\mu \nu}^{3}-2 \eta_{\mu \nu}^{3} N_{2} A_{\rho}^{2}\right), \\
j_{\rho}^{2} & =\frac{1}{4 \pi} \varepsilon^{\rho \lambda \mu \nu} \partial_{\lambda} B_{\mu \nu}^{2}+\xi_{\rho}^{2}-\Lambda \varepsilon^{\mu \nu \lambda \rho}\left(N_{3} B_{\mu \nu}^{3} N_{1} A_{\lambda}^{1}-\frac{1}{2} \eta_{\lambda}^{1} N_{3} B_{\mu \nu}^{3}-2 \eta_{\mu \nu}^{3} N_{1} A_{\lambda}^{1}\right) .
\end{aligned}
$$




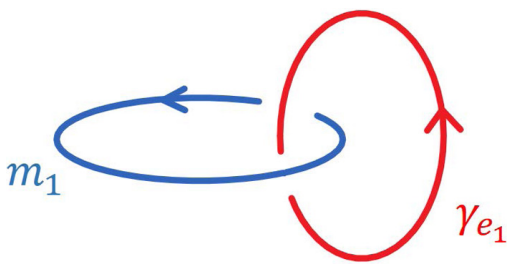

FIG. 1. Particle-loop braiding described by $B^{1} d A^{1}$ and $\Theta_{1}^{\mathrm{H}} . m_{1}$ is an elementary loop that carries unit gauge flux of $\mathbb{Z}_{N_{1}}$ gauge group. $\gamma_{e_{1}}$ is the closed trajectory of an elementary particle $e_{1}$ that carries unit gauge charge of $\mathbb{Z}_{N_{1}}$ gauge group.

The physical meaning of $\Sigma_{\mu \nu}^{3}$ is the current of loop while those of $j_{\lambda}^{1}$ and $j_{\rho}^{2}$ are the currents of particles of layers 1 and 2, respectively. In this manner, one can figure out the microscopic origins of particle and loop excitations. Substituting $\Sigma_{\mu \nu}^{3}, j_{\lambda}^{1}$, and $j_{\rho}^{2}$ into Lagrangian Eq. (2) and integrating out the Lagrange multiplier fields, we end up with

$$
\begin{aligned}
\mathcal{L}= & \frac{i N_{1}}{4 \pi} \varepsilon^{\mu \nu \lambda \rho} B_{\mu \nu}^{1} \partial_{\lambda} A_{\rho}^{1}+\frac{i N_{2}}{4 \pi} \varepsilon^{\mu \nu \lambda \rho} B_{\mu \nu}^{2} \partial_{\lambda} A_{\rho}^{2} \\
& +\frac{i N_{3}}{4 \pi} \varepsilon^{\mu \nu \lambda \rho} B_{\mu \nu}^{3} \partial_{\lambda} A_{\rho}^{3}+i N_{1} N_{2} N_{3} \Lambda \varepsilon^{\mu \nu \lambda \rho} A_{\mu}^{1} A_{\nu}^{2} B_{\lambda \rho}^{3} .
\end{aligned}
$$

Finally, we effectively obtain the TQFT action for a BR braiding: $S=\int \mathcal{L} d x d t \sim \int \sum_{i=1}^{3} \frac{N_{i}}{2 \pi} B^{i} d A^{i}+A^{1} A^{2} B^{3}$.

In this theory, the particle excitations are $e_{1}, e_{2}, e_{3}$, carrying gauge charges of $\mathbb{Z}_{N_{1}}, \mathbb{Z}_{N_{2}}, \mathbb{Z}_{N_{3}}$ and minimally couple to $A^{1}, A^{2}, A^{3}$. The loop excitations are $m_{1}, m_{2}, m_{3}$, carrying gauge fluxes of $\mathbb{Z}_{N_{1}}, \mathbb{Z}_{N_{2}}, \mathbb{Z}_{N_{3}}$, and minimally couple to $B^{1}, B^{2}, B^{3}$.

\section{B. Particle-loop braiding and $B d A$ term}

The particle-loop braiding is essentially a quantized Aharonov-Bohm effect in a discrete gauge theory where local interactions are completely screened and long-range statistical interactions lead to nontrivial braiding phases. Given a $\mathbb{Z}_{N_{1}}$ gauge subgroup, an elementary particle $e_{1}$ is braided around a static elementary loop $m_{1}$. The trajectory $\gamma_{e_{1}}$ and $m_{1}$ as a whole form a Hopf link, as shown in Fig. 1. The corresponding braiding phase is given by $\Theta_{i}^{\mathrm{H}}=\frac{2 \pi}{N_{i}}$ for unit Hopf linking number. The classification of equivalent trajectories of $e_{1}$ is essentially related to the mathematics of fundamental group and link homotopy, which was preliminarily introduced in the Supplemental Material of Ref. [45]. The corresponding TQFT is the following multicomponent $B F$ action $^{4}(F=d A, \wedge$ is omitted):

$$
S=\int \sum_{i=1}^{n} \frac{N_{i}}{2 \pi} B^{i} d A^{i},
$$

where $N_{i}$ are positive integers that specify the discrete gauge group $G$. $\left\{A^{i}=\sum_{\mu} A_{\mu}^{i} d x_{\mu}\right\}$ and $\left\{B^{i}=\frac{1}{2 !} \sum_{\mu \nu} B_{\mu \nu}^{i} d x_{\mu} d x_{\nu}\right\}$ are one-form and two-form compact $\mathbb{U}(1)$ gauge fields, respectively. Although one may expect a general matrix formalism $\sim K^{i j} B^{i} d A^{j}$, the matrix $K$ can always be sent to a

\footnotetext{
${ }^{4}$ In this paper, each summation is indicated by a $\sum$ symbol.
}

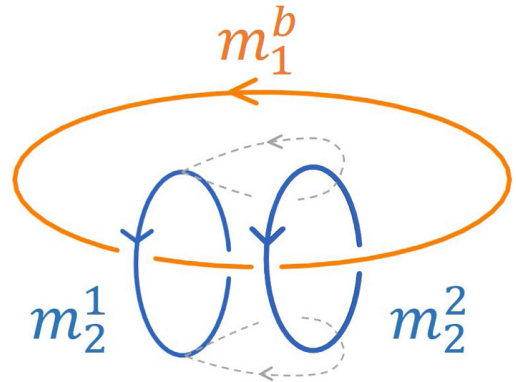

FIG. 2. Three-loop braiding process described by $A^{1} A^{2} d A^{2}$ and $\Theta_{2,2 \mid 1}^{3 \mathrm{~L}}$. In this case, the base loop $m_{1}^{b}$ carries the $\mathbb{Z}_{N_{1}}$ gauge flux; Both $m_{2}^{1}$ and $m_{2}^{2}$ carry the $\mathbb{Z}_{N_{2}}$ gauge flux.

diagonal matrix with positive elements via two independent $\mathbb{G} \mathbb{L}(n, \mathbb{Z})$ transformations. The two transformations, respectively, act on $B$ fields and $A$ fields (a relevant discussion on basis transformations can be found in Refs. [30,53]). The action Eq. (5) keeps invariant up to boundary terms under gauge transformations

$$
\begin{aligned}
& A^{i} \rightarrow A^{i}+d \chi^{i}, \\
& B^{i} \rightarrow B^{i}+d V^{i},
\end{aligned}
$$

where $\left\{\chi^{i}\right\}$ and $\left\{V^{i}\right\}$ are, respectively, zero-form and oneform compact $\mathbb{U}(1)$ gauge parameters with $\int d \chi \in 2 \pi \mathbb{Z}$ and $\int d V \in 2 \pi \mathbb{Z}$. Once these integrals are nonzero, the corresponding gauge transformations are said to be large. The coefficient quantization, i.e., $N_{1}$ is integral and is guaranteed by the invariance of the partition function $\mathcal{Z}=$ $\int \mathscr{D} B \mathscr{D} A e^{i \int \frac{N_{1}}{2 \pi} B d A}$ under large gauge transformations on any compact oriented manifold.

\section{Multiloop braiding and $A A d A, A A A A$ terms}

Next, we consider three-loop and four-loop braiding processes introduced in Sec. I. The minimal number of gauge subgroups is two: $G=\mathbb{Z}_{N_{1}} \times \mathbb{Z}_{N_{2}}$. For this gauge group, we consider a three-loop braiding process (Fig. 2) with braiding phase denoted as $\Theta_{2,2 \mid 1}^{3 \mathrm{~L}}$. The TQFT action for this three-loop braiding process is

$$
S=\int \sum_{i=1}^{2} \frac{N_{i}}{2 \pi} B^{i} d A^{i}+\frac{q_{122}}{(2 \pi)^{2}} A^{1} A^{2} d A^{2} .
$$

$S$ is invariant up to boundary terms under gauge transformations

$$
\begin{aligned}
& A^{i} \rightarrow A^{i}+d \chi^{i}, \\
& B^{1} \rightarrow B^{1}+d V^{1}+\frac{q_{122}}{2 \pi N_{1}} d \chi^{2} A^{2}, \\
& B^{2} \rightarrow B^{2}+d V^{2}-\frac{q_{122}}{2 \pi N_{2}} d \chi^{1} A^{2} .
\end{aligned}
$$

The coefficient $q_{122}$ is quantized and periodic: $q_{122}=\frac{k N_{1} N_{2}}{N_{12}}$, where $k \in \mathbb{Z}_{N_{12}}$ and $N_{12}$ is the greatest common divisor (GCD) of $N_{1}$ and $N_{2}$.

When $G=\mathbb{Z}_{N_{1}} \times \mathbb{Z}_{N_{2}} \times \mathbb{Z}_{N_{3}}$, we consider a three-loop braiding process (Fig. 3 ) associated with the braiding phase 


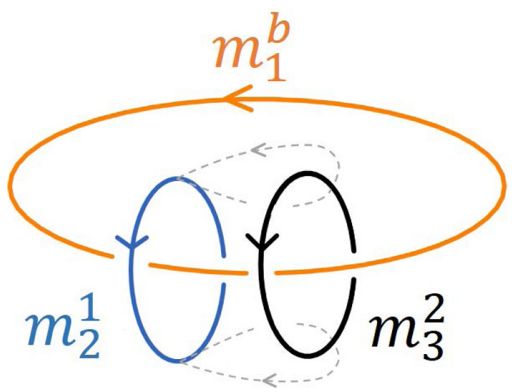

FIG. 3. Three-loop braiding process described by $A^{1} A^{2} d A^{3}$ and $\Theta_{2,3 \mid 1}^{3 \mathrm{~L}}$. In this case, the base loop $m_{1}^{b}$ carries the $\mathbb{Z}_{N_{3}}$ gauge flux, $m_{2}^{1}$ carries the $\mathbb{Z}_{N_{2}}$ gauge flux, and $m_{3}^{2}$ carries the $\mathbb{Z}_{N_{3}}$ gauge flux.

$\Theta_{2,3 \mid 1}^{3 \mathrm{~L}}$. The corresponding TQFT action is

$$
S=\int \sum_{i=1}^{3} \frac{N_{i}}{2 \pi} B^{i} d A^{i}+\frac{q_{123}}{(2 \pi)^{2}} A^{1} A^{2} d A^{3} .
$$

The gauge transformations are defined as

$$
\begin{aligned}
& A^{i} \rightarrow A^{i}+d \chi^{i}, \\
& B^{i} \rightarrow B^{i}+d V^{i}+\frac{q_{123}}{2 \pi N_{i}} \epsilon^{i j 3} d \chi^{j} A^{3},
\end{aligned}
$$

where $\epsilon$ is the Levi-Civita symbol with $\epsilon^{123}=-\epsilon^{213}=1$. The coefficient is $q_{123}=\frac{k N_{1} N_{2}}{N_{123}}$ where $k \in \mathbb{Z}_{N_{123}}$ and $N_{123}$ is the GCD of $N_{1}, N_{2}$, and $N_{3}$. When $G=\mathbb{Z}_{N_{1}} \times \mathbb{Z}_{N_{2}} \times \mathbb{Z}_{N_{3}} \times \mathbb{Z}_{N_{4}}$, four-loop braiding processes are realizable, in which four loops carry the unit gauge fluxes of $\mathbb{Z}_{N_{1}}, \mathbb{Z}_{N_{2}}, \mathbb{Z}_{N_{3}}, \mathbb{Z}_{N_{4}}$ gauge subgroups, respectively. The corresponding TQFT action is

$$
S=\int \sum_{i=1}^{4} \frac{N_{i}}{2 \pi} B^{i} d A^{i}+\frac{q_{1234}}{(2 \pi)^{3}} A^{1} A^{2} A^{3} A^{4} .
$$

The action Eq. (11) is invariant up to boundary terms under gauge transformations

$$
\begin{aligned}
A^{i} \rightarrow A^{i} & +d \chi^{i}, \\
B^{i} \rightarrow & B^{i}+d V^{i} \\
& -\frac{1}{2} \sum_{j, k, l} \frac{q_{1234}}{(2 \pi)^{2} N_{i}} \epsilon^{i j k l} A^{j} A^{k} \chi^{l} \\
& +\frac{1}{2} \sum_{j, k, l} \frac{q_{1234}}{(2 \pi)^{2} N_{i}} \epsilon^{i j k l} A^{j} \chi^{k} d \chi^{l} \\
& +\frac{1}{6} \sum_{j, k, l} \frac{q_{1234}}{(2 \pi)^{2} N_{i}} \epsilon^{i j k l} \chi^{j} d \chi^{k} d \chi^{l},
\end{aligned}
$$

where $\epsilon$ is the Levi-Civita symbol with $\epsilon^{1234}=-\epsilon^{1324}=$ 1. The coefficient is $q_{1234}=\frac{k N_{1} N_{2} N_{3} N_{4}}{N_{1234}}$, where $k \in \mathbb{Z}_{N_{1234}}$ and $N_{1234}$ is the GCD of $N_{1}, N_{2}, N_{3}$, and $N_{4}$. Until now, we have only reviewed gauge transformations in this review section. It is hard to visualize four-loop braiding in three-dimensional real space. We recommend Fig. 6 of Ref. [28].

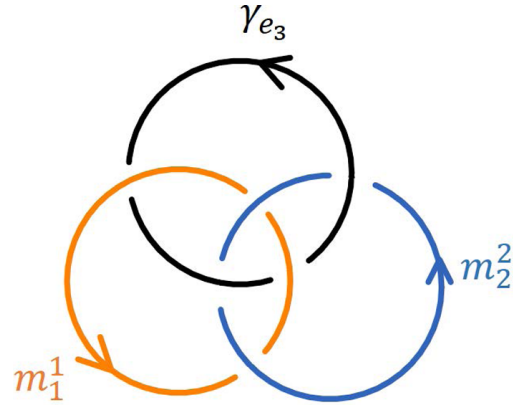

FIG. 4. Borromean rings braiding described by $A^{1} A^{2} B^{3}$ and $\Theta_{1,2 \mid 3}^{\mathrm{BR}} . m_{1}^{1}$ and $m_{2}^{2}$, respectively, carry unit gauge flux of $\mathbb{Z}_{N_{1}}$ and $\mathbb{Z}_{N_{2}}$ gauge subgroup. $e_{3}$, carrying unit gauge charge of $\mathbb{Z}_{N_{3}}$ gauge subgroup, moves around $m_{1}^{1}$ and $m_{2}^{2}$, such that the trajectory of $e_{3}$ (denoted as $\gamma_{e_{3}}$ ) and other two loops form a Borromean ring link.

\section{Borromean rings braiding and $A A B$ term}

Last, we consider the particle-loop-loop braiding or BR braiding with its braiding phase denoted as $\Theta_{i, j \mid k}^{\mathrm{BR}}$ [45]. Likewise, the input data of BR braiding are Abelian gauge group $G=\prod_{i=1}^{n} \mathbb{Z}_{N_{i}}$ but with $n \geqslant 3$, such that all particles and loops can be labeled by gauge charges and gauge fluxes in a specific gauge subgroup $\mathbb{Z}_{N_{i}}$. This braiding is beyond Dijkgraaf-Witten gauge theory classification $\mathcal{H}^{4}(G, U(1))$. The latter only includes braiding phases of particle-loop braidings and multi-loop braidings. By further taking BR braiding into account, we need to study proper combinations of all braidings together to exhaust all topological orders.

The corresponding topological term of BR braidings is $A^{i} A^{j} B^{k}$. For example, consider a BR braiding process shown in Fig. 4, the corresponding TQFT action is

$$
S=\int \sum_{i=1}^{3} \frac{N_{i}}{2 \pi} B^{i} d A^{i}+\frac{p_{12,3}}{(2 \pi)^{2}} A^{1} A^{2} B^{3},
$$

where $p_{12,3}=\frac{l_{12,3} N_{1} N_{2} N_{3}}{N_{123}}, l_{12,3} \in \mathbb{Z}_{N_{123}}, N_{123}$ is the GCD of $N_{1}$, $N_{2}$ and $N_{3}$. Ref. [45] points out that $l_{12,3}=-l_{21,3}$ and $l_{i j, k}=0$ if any of the two indices are same. The quantization of $p_{12,3}$ is due to the large gauge invariance. The gauge transformations for (13) are

$$
\begin{aligned}
& A^{1} \rightarrow A^{1}+d \chi^{1}, \\
& A^{2} \rightarrow A^{2}+d \chi^{2}, \\
& A^{3} \rightarrow A^{3}+d \chi^{3}+X^{3}, \\
& B^{1} \rightarrow B^{1}+d V^{1}+Y^{1}, \\
& B^{2} \rightarrow B^{2}+d V^{2}+Y^{2}, \\
& B^{3} \rightarrow B^{3}+d V^{3},
\end{aligned}
$$

where

$$
\begin{aligned}
X^{3}= & -\frac{p_{12,3}}{2 \pi N_{3}}\left(\chi^{1} A^{2}+\frac{1}{2} \chi^{1} d \chi^{2}\right) \\
& +\frac{p_{12,3}}{2 \pi N_{3}}\left(\chi^{2} A^{1}+\frac{1}{2} \chi^{2} d \chi^{1}\right), \\
Y^{1}= & -\frac{p_{12,3}}{2 \pi N_{1}}\left(\chi^{2} B^{3}-A^{2} V^{3}+\chi^{2} d V^{3}\right), \\
Y^{2}= & \frac{p_{12,3}}{2 \pi N_{2}}\left(\chi^{1} B^{3}-A^{1} V^{3}+\chi^{1} d V^{3}\right) .
\end{aligned}
$$


$X^{3}, Y^{1}$ and $Y^{2}$ are so-called shift terms. The Dirac quantization of $A^{3}, B^{1}$ and $B^{2}$ requires that $\frac{1}{2 \pi} \int d X^{3} \in \mathbb{Z}, \frac{1}{2 \pi} \int d Y^{1} \in$ $\mathbb{Z}$ and $\frac{1}{2 \pi} \int d Y^{2} \in \mathbb{Z}$. The $\mathbb{Z}_{N_{i}}$ cyclic group structures are encoded in the cyclic Wilson integrals of $A^{1}, A^{2}$ and $B^{3}$ respectively: $\oint A^{1} \in \frac{2 \pi}{N_{1}} \mathbb{Z}_{N_{1}}, \oint A^{2} \in \frac{2 \pi}{N_{2}} \mathbb{Z}_{N_{2}}$ and $\oint B^{3} \in \frac{2 \pi}{N_{3}} \mathbb{Z}_{N_{3}}$.

\section{COMPATIBLE BRAIDING PROCESSES}

Since we have reviewed the correspondence between topological terms, braiding processes and braiding phases, we may naively think that we can design a system which can support arbitrary combinations of braiding processes. However, this is not true. In other words, there are illegitimate combinations in which the braiding processes are mutually incompatible. Such incompatibility reveals that some topological terms are forbidden to form a legitimate TQFT action.

In this section, we will compute the sets of compatible braiding phases for different gauge groups, from which the compatible braiding processes can be read out. For this purpose, we seek for a legitimate TQFT action consisting of as many topological terms as possible for a given gauge group.

In order to verify a TQFT action is legitimate or not, we need to take care of the following aspects. First of all, a legitimate TQFT action should be invariant up to boundary terms under proper gauge transformations. Second, the gauge transformations are required to preserve the $\mathbb{Z}_{N_{i}}$ cyclic group structure. This requirement means that, if the $\mathbb{Z}_{N_{j}}$ cyclic group structure is encoded in $\oint A^{i} \in \frac{2 \pi}{N_{i}} \mathbb{Z}_{N_{i}}\left(\oint B^{i} \in \frac{2 \pi}{N_{i}} \mathbb{Z}_{N_{i}}\right)$, $A^{i}\left(B^{i}\right)$ must have the standard gauge transformation: $A^{i} \rightarrow$ $A^{i}+d \chi^{i}\left(B^{i} \rightarrow B^{i}+d V^{i}\right)$ such that the Wilson integrals of $A^{i}\left(B^{i}\right)$ are gauge-invariant. $\chi^{i}$ and $V^{i}$ are zero-form and 1form compact $\mathbb{U}(1)$ gauge parameters with $\int d \chi^{i} \in 2 \pi \mathbb{Z}$ and $\int d V^{i} \in 2 \pi \mathbb{Z}$ respectively. From the perspective of microscopic origins (Sec. II A), if $A^{i}$ has the above standard gauge transformation, the $i$-th layer condensate must be a charge- $N_{i}$ boson condensate that higgses the Wilson integrals of $A^{i}$ down to $\mathbb{Z}_{N_{i}}$, and $B^{i}$ comes from the duality transformation shown in Sec. II A and Appendix A; likewise, if $B^{i}$ has the above standard gauge transformation, the $i$-th layer condensate must be a charge- $N_{i}$ vortexline condensate that higgses the Wilson integrals of $B^{i}$ down to $\mathbb{Z}_{N_{i}}$, and $A^{i}$ comes from the duality transformation shown in Sec. II A and Appendix A. In summary, in a legitimate action, at least one of gauge fields ( $A^{i}$ and $B^{i}$ ), for a given $i$, should have the above standard gauge transformations and thus have the $\mathbb{Z}_{N_{i}}$ quantized Wilson integrals. For example, see Eqs. (6), (8), (10), (12), and (14).

Next, the gauge transformation of each gauge field should respect the Dirac quantization. Last but not least, a legitimate TQFT action should consist of topological terms with nontrivial coefficients. If the coefficient of a topological term is identical to 0 , otherwise the action cannot be gauge invariant under gauge transformations, this topological term is actually incompatible with others in the action.

$$
\text { A. } \boldsymbol{G}=\mathbb{Z}_{N_{1}} \text { and } \boldsymbol{G}=\mathbb{Z}_{N_{1}} \times \mathbb{Z}_{N_{2}}
$$

When $G=\mathbb{Z}_{N_{1}}$, the elementary particle (loop) is $e_{1}\left(m_{1}\right)$ carrying unit gauge charge (flux) of $\mathbb{Z}_{N_{1}}$ gauge group. The only nontrivial braiding process in this case is the particle- loop braiding described by

$$
S=\int \frac{N_{1}}{2 \pi} B^{1} d A^{1} .
$$

The gauge transformations for Eq. (16) are

$$
\begin{aligned}
& A^{1} \rightarrow A^{1}+d \chi^{1}, \\
& B^{1} \rightarrow B^{1}+d V^{1} .
\end{aligned}
$$

The braiding phase of this particle-loop braiding is

$$
\Theta_{1}^{\mathrm{H}}=\frac{2 \pi}{N_{1}} .
$$

When $G=\mathbb{Z}_{N_{1}} \times \mathbb{Z}_{N_{2}}$, the elementary particles (loops) are denoted as $e_{1}$ and $e_{2}\left(m_{1}\right.$ and $\left.m_{2}\right)$ carrying unit gauge charge (flux) of $\mathbb{Z}_{N_{1}}$ and $\mathbb{Z}_{N_{2}}$ gauge subgroups, respectively. Beside particle-loop braidings, three-loop braidings can be supported. There are four kinds of three-loop braiding phases in this case, described by two linearly independent $A A d A$ terms:

(1) $\Theta_{2,2 \mid 1}^{3 \mathrm{~L}}: m_{l}^{b}, m_{j}^{1}$, and $m_{k}^{2}$, respectively, carry unit gauge fluxes of $\mathbb{Z}_{N_{1}}, \mathbb{Z}_{N_{2}}$, and $\mathbb{Z}_{N_{2}}$ gauge subgroups.

(2) $\Theta_{1,2 \mid 2}^{3 \mathrm{~L}}: m_{l}^{b}, m_{j}^{1}$, and $m_{k}^{2}$ respectively, carry unit gauge fluxes of $\mathbb{Z}_{N_{2}}, \mathbb{Z}_{N_{1}}$ and $\mathbb{Z}_{N_{2}}$ gauge subgroups.

(3) $\Theta_{1,1 \mid 2}^{3 \mathrm{~L}}: m_{l}^{b}, m_{j}^{1}$, and $m_{k}^{2}$, respectively, carry unit gauge fluxes of $\mathbb{Z}_{N_{2}}, \mathbb{Z}_{N_{1}}$, and $\mathbb{Z}_{N_{1}}$ gauge subgroups.

(4) $\Theta_{2,111}^{3 \mathrm{~L}}: m_{l}^{b}, m_{j}^{1}$, and $m_{k}^{2}$, respectively, carry unit gauge fluxes of $\mathbb{Z}_{N_{1}}, \mathbb{Z}_{N_{2}}$, and $\mathbb{Z}_{N_{1}}$ gauge subgroups.

The first two braiding processes are described by $A^{1} A^{2} d A^{2}$ and the remainder by $A^{2} A^{1} d A^{1}$. All these three-loop braidings and particle-loop braidings are compatible, described by a TQFT action

$$
\begin{aligned}
S= & \int \sum_{i=1}^{2} \frac{N_{i}}{2 \pi} B^{i} d A^{i} \\
& +\frac{q_{122}}{(2 \pi)^{2}} A^{1} A^{2} d A^{2}+\frac{q_{211}}{(2 \pi)^{2}} A^{2} A^{1} d A^{1},
\end{aligned}
$$

where $q_{122}=\frac{k N_{1} N_{2}}{N_{12}}, k \in \mathbb{Z}_{N_{12}}$, and $q_{211}=\frac{k^{\prime} N_{2} N_{1}}{N_{12}}, k^{\prime} \in \mathbb{Z}_{N_{12}}$. $N_{12}$ is the GCD of $N_{1}$ and $N_{2}$. The gauge transformations for Eq. (19) are

$$
\begin{aligned}
& A^{i} \rightarrow A^{i}+d \chi^{i}, \\
& B^{1} \rightarrow B^{1}+d V^{1}+\frac{q_{122}}{(2 \pi) N_{1}} d \chi^{2} A^{2}-\frac{q_{211}}{(2 \pi) N_{1}} d \chi^{2} A^{1}, \\
& B^{2} \rightarrow B^{2}+d V^{2}-\frac{q_{122}}{(2 \pi) N_{2}} d \chi^{1} A^{2}+\frac{q_{211}}{(2 \pi) N_{2}} d \chi^{1} A^{1} .
\end{aligned}
$$

The phases of particle-loop braidings and three-loop braidings are

$$
\begin{gathered}
\Theta_{i}^{\mathrm{H}}=\frac{2 \pi}{N_{i}}, \\
\Theta_{2,2 \mid 1}^{3 \mathrm{~L}}=\frac{4 \pi q_{122}}{N_{1} N_{2}}, \\
\Theta_{1,2 \mid 2}^{3 \mathrm{~L}}=-\frac{2 \pi q_{122}}{N_{1} N_{2}}, \\
\Theta_{1,1 \mid 2}^{3 \mathrm{~L}}=\frac{4 \pi q_{211}}{N_{1} N_{2}}, \\
\Theta_{2,1 \mid 1}^{3 \mathrm{~L}}=-\frac{2 \pi q_{211}}{N_{1} N_{2}} .
\end{gathered}
$$


Legitimate TQFT actions for $G=\mathbb{Z}_{N_{1}}$ and $G=\mathbb{Z}_{N_{1}} \times \mathbb{Z}_{N_{2}}$ are summarized in Table I. The sets of compatible braiding phases for $G=\mathbb{Z}_{N_{1}}$ and $G=\prod_{i=1}^{2} \mathbb{Z}_{N_{i}}$ are, respectively,

$$
\left\{\Theta_{1}^{\mathrm{H}}=\frac{2 \pi}{N_{1}}\right\}_{\mathbb{Z}_{N_{1}}}
$$

and

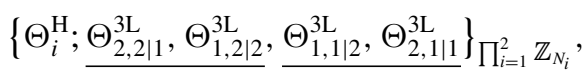

where the underlines denote the linear dependence of two braiding phases, for example, $\Theta_{2,2 \mid 1}^{3 \mathrm{~L}}, \Theta_{1,2 \mid 2}^{3 \mathrm{~L}}$ indicates that $\Theta_{2,2 \mid 1}^{3 \mathrm{~L}}=-2 \cdot \Theta_{1,2 \mid 2}^{3 \mathrm{~L}}$.

$$
\text { B. } \boldsymbol{G}=\mathbb{Z}_{N_{1}} \times \mathbb{Z}_{N_{2}} \times \mathbb{Z}_{N_{3}}
$$

When $G=\mathbb{Z}_{N_{1}} \times \mathbb{Z}_{N_{2}} \times \mathbb{Z}_{N_{3}}$, the elementary particles (loops) are $e_{1}, e_{2}$, and $e_{3}\left(m_{1}, m_{2}\right.$, and $\left.m_{3}\right)$ carrying unit gauge charges (fluxes) of $\mathbb{Z}_{N_{1}}, \mathbb{Z}_{N_{2}}$, and $\mathbb{Z}_{N_{3}}$ gauge subgroups, respectively. In addition to the particle-loop braidings and three-loop braidings discussed in Sec. III A, BR braidings described by $A A B$ terms and three-loop braidings described by $A^{m} A^{n} d A^{o}$ terms ( $m, n, o$ are mutually different) are realizable. For the reason BR braiding is absent when $G=\prod_{i=1}^{2} \mathbb{Z}_{N_{i}}$, one can refer to Sec. IV A.

The compatibility issues of $G=\prod_{i=1}^{3} \mathbb{Z}_{N_{i}}$ are discussed as follows. First, if we neglect BR braidings, all particle-loop braidings and three-loop braidings are compatible, we can write a legitimate TQFT action for all root braiding processes except BR braidings:

$$
\begin{aligned}
& \int \sum_{i=1}^{3} \frac{N_{i}}{2 \pi} B^{i} d A^{i}+\frac{q_{122}}{(2 \pi)^{2}} A^{1} A^{2} d A^{2}+\frac{q_{211}}{(2 \pi)^{2}} A^{2} A^{1} d A^{1} \\
& +\frac{q_{133}}{(2 \pi)^{2}} A^{1} A^{3} d A^{3}+\frac{q_{311}}{(2 \pi)^{2}} A^{3} A^{1} d A^{1}+\frac{q_{233}}{(2 \pi)^{2}} A^{2} A^{3} d A^{3} \\
& +\frac{q_{322}}{(2 \pi)^{2}} A^{3} A^{2} d A^{2}+\frac{q_{123}}{(2 \pi)^{2}} A^{1} A^{2} d A^{3}+\frac{q_{231}}{(2 \pi)^{2}} A^{2} A^{3} d A^{1},
\end{aligned}
$$

where $q_{i j k}=\frac{l N_{i} N_{j}}{N_{i j}}, l \in \mathbb{Z}_{N_{i j k}}, N_{i j}\left(N_{i j k}\right)$ is the GCD of $N_{i}$, $N_{j}$ (and $N_{k}$ ). Next, we consider a BR braiding, say, $\Theta_{1,2 \mid 3}^{\mathrm{BR}}$ described by an $A^{1} A^{2} B^{3}$ term. All particle-loop braidings are compatible with this BR braiding. However, among three-loop braiding phases, only $\Theta_{2,2 \mid 1}^{3 \mathrm{~L}}, \Theta_{1,2 \mid 2}^{3 \mathrm{~L}}, \Theta_{1,1 \mid 2}^{3 \mathrm{~L}}$, and $\Theta_{2,1 \mid 1}^{3 \mathrm{~L}}$ are compatible with $\Theta_{1,2 \mid 3}^{\mathrm{BR}}$. Other $\Theta_{i, j \mid k}^{3 \mathrm{~L}}$ 's with one index equal to 3 are not compatible with $\Theta_{1,2 \mid 3}^{\mathrm{BR}}$. The reasons for this incompatibility between three-loop braidings and $\mathrm{BR}$ braidings are discussed in Secs. IV C-IV E. The total TQFT action for $\Theta_{1,2 \mid 3}^{\mathrm{BR}}$ and its compatible braiding processes is

$$
\begin{aligned}
& \int \sum_{i=1}^{3} \frac{N_{i}}{2 \pi} B^{i} d A^{i}+\frac{p_{12,3}}{(2 \pi)^{2}} A^{1} A^{2} B^{3} \\
& +\frac{q_{122}}{(2 \pi)^{2}} A^{1} A^{2} d A^{2}+\frac{q_{211}}{(2 \pi)^{2}} A^{2} A^{1} d A^{1},
\end{aligned}
$$

where $p_{12,3}=\frac{l N_{1} N_{2} N_{3}}{N_{123}}, l \in \mathbb{Z}_{N_{123}}$, and $q_{i j j}=\frac{l^{\prime} N_{i} N_{j}}{N_{i j}}, l^{\prime} \in \mathbb{Z}_{N_{i j}}$. Furthermore, if we consider two different BR braidings, we find that it is impossible to write a legitimate TQFT action which contains two different $A A B$ terms. The reason is explained in Sec. IV B. Nevertheless, if the gauge group is $G=\prod_{i=1}^{n} \mathbb{Z}_{N_{i}}$ with $n \geqslant 4$, legitimate TQFT actions for two different BR braidings are possible, which is detailed in Sec. III C.

By checking all combinations of braiding processes with the criteria of compatibility, we summarized all legitimate TQFT actions for $G=\mathbb{Z}_{N_{1}} \times \mathbb{Z}_{N_{2}} \times \mathbb{Z}_{N_{3}}$ and corresponding gauge transformations in Table II. The sets of compatible braiding phases for $G=\prod_{i=1}^{3} \mathbb{Z}_{N_{i}}$ can be summarized as

$$
\begin{aligned}
& \left\{\Theta_{i}^{\mathrm{H}} ; \Theta_{j, j \mid i}^{3 \mathrm{~L}}, \Theta_{i, j \mid j}^{3 \mathrm{~L}}, \underline{\Theta_{2,3 \mid 1}^{3 \mathrm{~L}}, \Theta_{1,3 \mid 2}^{3 \mathrm{~L}},}, \underline{\left.\Theta_{3,1 \mid 2}^{3 \mathrm{~L}}, \Theta_{2,1 \mid 3}^{3 \mathrm{~L}}\right\}_{\prod_{i=1}^{3} \mathbb{Z}_{N_{i}}},}\right. \\
& \left\{\Theta_{i}^{\mathrm{H}} ; \Theta_{3,3 \mid 2}^{3 \mathrm{~L}}, \Theta_{2,3 \mid 3}^{3 \mathrm{~L}}, \frac{\left.\Theta_{2,2 \mid 3}^{3 \mathrm{~L}}, \Theta_{3,2 \mid 2}^{3 \mathrm{~L}} ; \Theta_{2,3 \mid 1}^{\mathrm{BR}}\right\}_{\prod_{i=1}^{3} \mathbb{Z}_{N_{i}}},}{\left\{\Theta_{i}^{\mathrm{H}} ; \Theta_{1,1 \mid 3}^{3 \mathrm{~L}}, \Theta_{3,1 \mid 1}^{3 \mathrm{~L}}, \Theta_{3,3 \mid 1}^{3 \mathrm{~L}}, \Theta_{1,3 \mid 3}^{3 \mathrm{~L}} ; \Theta_{3,1 \mid 2}^{\mathrm{BR}}\right\}_{\prod_{i=1}^{3} \mathbb{Z}_{N_{i}}},}\right.
\end{aligned}
$$

and

$$
\left\{\Theta_{i}^{\mathrm{H}} ; \Theta_{2,2 \mid 1}^{3 \mathrm{~L}}, \Theta_{1,2 \mid 2}^{3 \mathrm{~L}}, \Theta_{1,1 \mid 2}^{3 \mathrm{~L}}, \Theta_{2,1 \mid 1}^{3 \mathrm{~L}} ; \Theta_{1,2 \mid 3}^{\mathrm{BR}}\right\}_{\prod_{i=1}^{3} \mathbb{Z}_{N_{i}}},
$$

where the underlines denote the linear dependence between two compatible braiding phases: $\Theta_{3,3 \mid 2}^{3 \mathrm{~L}}=-2 \times \Theta_{2,3 \mid 3}^{3 \mathrm{~L}}$, $\Theta_{2,3 \mid 1}^{3 \mathrm{~L}}=-\Theta_{1,3 \mid 2}^{3 \mathrm{~L}}$, etc.

$$
\text { C. } G=\mathbb{Z}_{N_{1}} \times \mathbb{Z}_{N_{2}} \times \mathbb{Z}_{N_{3}} \times \mathbb{Z}_{N_{4}}
$$

Similar to the case in Sec. III B, particle-loop braidings and multiloop braidings are compatible with each other when $G=$ $\prod_{i=1}^{4} \mathbb{Z}_{N_{i}}$. The TQFT action for them is

$$
\begin{aligned}
& \int \sum_{i=1}^{4} \frac{N_{i}}{2 \pi} B^{i} d A^{i}+\sum_{i<j}\left[\frac{q_{i j j}}{(2 \pi)^{2}} A^{i} A^{j} d A^{j}+\frac{q_{j i i}}{(2 \pi)^{2}} A^{j} A^{i} d A^{i}\right] \\
+ & \sum_{i<j<k}\left[\frac{q_{i j k}}{(2 \pi)^{2}} A^{i} A^{j} d A^{k}+\frac{q_{j k i}}{(2 \pi)^{2}} A^{j} A^{k} d A^{i}\right] \\
+ & \frac{q_{1234}}{(2 \pi)^{3}} A^{1} A^{2} A^{3} A^{4} .
\end{aligned}
$$

The set of compatible braiding phases for Eq. (34) is

$$
\begin{aligned}
& \left\{\Theta_{i}^{\mathrm{H}} ; \Theta_{j, j \mid i}^{3 \mathrm{~L}}, \Theta_{i, j \mid j}^{3 \mathrm{~L}}, \Theta_{i, i \mid j}^{3 \mathrm{~L}}, \Theta_{j, i \mid i}^{3 \mathrm{~L}},\right.
\end{aligned}
$$

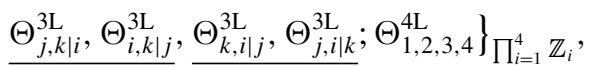

where $i<j<k$ and $\{i, j, k\} \subset\{1,2,3,4\}$.

When $G=\prod_{i=1}^{4} \mathbb{Z}_{i}$, if we take BR braidings into account, we need to treat them carefully. If these BR braidings involve only one kind of elementary particles, say, $e_{1}$, corresponding to $A^{2} A^{3} B^{1}, A^{2} A^{4} B^{1}$ and $A^{3} A^{4} B^{1}$ terms, the legitimate TQFT action is

$$
\begin{aligned}
\int & \sum_{i=1}^{4} \frac{N_{i}}{2 \pi} B^{i} d A^{i}+\sum_{i, j \neq 1} \frac{q_{i j j}}{(2 \pi)^{2}} A^{i} A^{j} d A^{j} \\
+ & \frac{q_{234}}{(2 \pi)^{2}} A^{2} A^{3} d A^{4}+\frac{q_{342}}{(2 \pi)^{2}} A^{3} A^{4} d A^{2} \\
+ & \frac{p_{23,1}}{(2 \pi)^{2}} A^{2} A^{3} B^{1}+\frac{p_{24,1}}{(2 \pi)^{2}} A^{2} A^{4} B^{1}+\frac{p_{34,1}}{(2 \pi)^{2}} A^{3} A^{4} B^{1}
\end{aligned}
$$


corresponding to the set of compatible braiding phases

$$
\begin{aligned}
\left\{\Theta_{i}^{\mathrm{H}} ;\right. & \frac{\Theta_{s, s \mid r}^{3 \mathrm{~L}}, \Theta_{r, s \mid s}^{3 \mathrm{~L}}, \Theta_{3,4 \mid 2}^{3 \mathrm{~L}}, \Theta_{2,4 \mid 3}^{3 \mathrm{~L}},}{\left.\Theta_{4,2 \mid 3}^{3 \mathrm{~L}}, \Theta_{3,2 \mid 4}^{3 \mathrm{~L}} ; \Theta_{4,2 \mid 3}^{\mathrm{BR}} ; \Theta_{2,4 \mid 1}^{\mathrm{BR}} ; \Theta_{3,4 \mid 1}^{\mathrm{BR}}\right\}_{\prod_{i=1}^{4} \mathbb{Z}_{i}},},
\end{aligned}
$$

with $r \neq 1$ and $s \neq 1$. In other words, any multiloop braiding which involves elementary loop $m_{1}$ is incompatible with BR braidings which involve elementary particle $e_{1}$.

If we consider BR braidings which involve two kinds of elementary particles, e.g., $e_{1}$ and $e_{2}$, the legitimate TQFT action is

$$
\begin{aligned}
& \int \sum_{i=1}^{4} \frac{N_{i}}{2 \pi} B^{i} d A^{i}+\frac{q_{344}}{(2 \pi)^{2}} A^{3} A^{4} d A^{4}+\frac{q_{433}}{(2 \pi)^{2}} A^{4} A^{3} d A^{3} \\
& +\frac{p_{34,1}}{(2 \pi)^{2}} A^{3} A^{4} B^{1}+\frac{p_{34,2}}{(2 \pi)^{2}} A^{3} A^{4} B^{2},
\end{aligned}
$$

which excludes multiloop braidings which involve elementary loops $m_{1}$ and $m_{2}$. The compatible braiding phases form a set:

$$
\left\{\Theta_{i}^{\mathrm{H}} ; \underline{\Theta_{4,4 \mid 3}^{3 \mathrm{~L}}, \Theta_{3,4 \mid 4}^{3 \mathrm{~L}}}, \underline{\Theta_{3,3 \mid 4}^{3 \mathrm{~L}}, \Theta_{4,3 \mid 3}^{3 \mathrm{~L}}} ; \Theta_{3,4 \mid 1}^{\mathrm{BR}} ; \Theta_{3,4 \mid 2}^{\mathrm{BR}}\right\}_{\prod_{i=1}^{4} \mathbb{Z}_{N_{i}}} .
$$

Notice that when we write down a BR braiding which involves one kind of elementary particles, we have four choices since there are four distinct gauge subgroups. If we consider two BR braidings with two kinds of elementary particles, we have six different combinations of these two different elementary particles. Therefore, there are total $1+4+6=11$ legitimate TQFT actions, i.e., 11 different sets of compatible braiding phases, when $G=\prod_{i=1}^{4} \mathbb{Z}_{N_{i}}$. Due to the limiting space of page, Table III only list three examples of TQFT actions and their gauge transformations. By properly reassigning the indices of the TQFT actions listed in Table III, we can construct all legitimate TQFT actions and corresponding gauge transformations. One can find details in Appendix B and Table IV therein.

\section{Results for general gauge groups}

In fact, our previous discussions can be easily generalized to the case where the number of $\mathbb{Z}_{N_{i}}$ subgroups are arbitrary. As we can see in the previous text, compatibility is always guaranteed for particle-loop braidings and multiloop braidings or particle-loop braidings and BR braidings. The general rule of incompatibility is that if two braiding processes (multiloop braiding and BR braiding, or two different BR braidings) involve particles and loops that carry gauge charge and gauge fluxes of the same $\mathbb{Z}_{N_{i}}$ gauge subgroup, these two braiding processes are incompatible with each other. In the language of TQFT, a $\mathbb{Z}_{N_{i}}$ gauge subgroup is associated with a topological term $B^{i} d A^{i}$. In a legitimate TQFT action, given the $B F$ term $B^{i} d A^{i}$, only $A^{i}$ or $B^{i}$, not both, can appear in the twisted terms (i.e., $A A d A, A A A A$, and $A A B$ ). Otherwise, the TQFT action would be illegitimate, e.g., violating the gauge invariance.

Viewed from the microscopic origin of topological terms, the rule of incompatibility is natural. Each layer of condensate (corresponding to each $\mathbb{Z}_{N_{i}}$ gauge subgroup) is either in the charge condensation or in the vortexline condensation, never both. The $\mathbb{Z}_{N_{i}}$ charge condensation implies particles that carry gauge charge $e_{i}$ (corresponding to $A^{i}$ ), while the
$\mathbb{Z}_{N_{i}}$ string condensation results in loops that carry gauge flux $m_{i}$ (corresponding to $B^{i}$ ). If a multiloop braiding or BR braiding involves the $\mathbb{Z}_{N_{i}}$ particle, it is incompatible with other braiding process that involves the $\mathbb{Z}_{N_{i}}$ loop, and vice versa.

\section{INCOMPATIBLE BRAIDING PROCESSES}

In Sec. III, we discuss the compatible braiding processes in different cases. We find that, when the gauge group is given, the set of compatible braiding processes is only a subset of \{all possible root braiding processes\}. This means that some braiding processes are mutually incompatible. For example, when $G=\prod_{i=1}^{3} \mathbb{Z}_{i}$ (see Sec. III B), a set of compatible braiding phases is $\left\{\Theta_{i}^{\mathrm{H}} ; \Theta_{2,2 \mid 1}^{3 \mathrm{~L}}, \Theta_{1,2 \mid 2}^{3 \mathrm{~L}}, \Theta_{1,1 \mid 2}^{3 \mathrm{~L}}, \Theta_{2,1 \mid 1}^{3 \mathrm{~L}} ; \Theta_{1,2 \mid 3}^{\mathrm{BR}}\right\}_{\prod_{i=1}^{3} \mathbb{Z}_{N_{i}}}$. We can see that, among all possible root braiding phases, $\Theta_{i, j \mid k}^{3 \mathrm{~L}}$ with one index equal to $3, \Theta_{2,3 \mid 1}^{\mathrm{BR}}$ and $\Theta_{3,1 \mid 2}^{\mathrm{BR}}$ are excluded from this set, because they are not compatible with $\Theta_{1,2 \mid 3}^{\mathrm{BR}}$. In this section, we will demonstrate several examples of such incompatibility between braiding processes via the TQFT perspective. The basic idea is if an action consists of topological terms corresponding to incompatible braiding processes, it would never be a legitimate TQFT theory.

\section{A. Absence of BR braidings when $G=\prod_{i=1}^{2} \mathbb{Z}_{i}$}

In Sec. II D, we review the BR braiding in which a particle carrying unit $\mathbb{Z}_{N_{3}}$ gauge charge moves around two loops which carry unit $\mathbb{Z}_{N_{1}}$ and $\mathbb{Z}_{N_{2}}$ gauge fluxes, respectively. Such a braiding process is only possible when there are three distinct $\mathbb{Z}_{N_{i}}$ gauge subgroups. It is natural to ask: Why it is impossible when there are only two $\mathbb{Z}_{N_{i}}$ gauge subgroups, i.e., $G=\prod_{i=1}^{2} \mathbb{Z}_{N_{i}}$ ? Without loss of generality, we can consider the following braiding process: a particle $e_{2}$ carrying unit $\mathbb{Z}_{N_{2}}$ gauge charge moves around two loops $m_{1}$ and $m_{2}$ which carry unit $\mathbb{Z}_{N_{1}}$ and $\mathbb{Z}_{N_{2}}$ gauge fluxes, respectively, such that the trajectory of $e_{1}$ and two loops form a BR link. One may naively think that this braiding process, along with its braiding phase $\Theta_{12,2}^{\mathrm{BR}}$, is described by the topological term $A^{1} A^{2} B^{2}$, analogous to the case in Sec. II D. The corresponding TQFT action should be

$$
S=\int \sum_{i=1}^{2} \frac{N_{i}}{2 \pi} B^{i} d A^{i}+\frac{p}{(2 \pi)^{2}} A^{1} A^{2} B^{2} .
$$

However, the action Eq. (40) is not a legitimate TQFT action because we cannot find gauge transformations which respects gauge invariance and $\mathbb{Z}_{N}$ cyclic group structure simultaneously. We are going to illustrate this in details.

In the action Eq. (40), $B^{1}$ serves as a Lagrange multiplier imposing a local constraint $d A^{1}=0$. This means that the gauge transformation of $A^{1}$ is $A^{1} \rightarrow A^{1}+d \chi^{1}$. Thus, the $\mathbb{Z}_{N_{1}}$ cyclic group structure is encoded in $\oint A^{i} \in \frac{2 \pi}{N_{i}} \mathbb{Z}_{N_{i}}$. The $\mathbb{Z}_{N_{2}}$ cyclic group structure requires that at least one of $A^{2} \rightarrow$ $A^{2}+d \chi^{2}$ and $B^{2} \rightarrow B^{2}+d V^{2}$ holds.

First, we assume that the gauge transformations are

$$
\begin{aligned}
& A^{i} \rightarrow A^{i}+d \chi^{i}, \\
& B^{i} \rightarrow B^{i}+d V^{i}+Y^{i},
\end{aligned}
$$


TABLE IV. General expressions of compatible braiding phases, TQFT actions, and gauge transformations when $G=\prod_{i=1}^{4} \mathbb{Z}_{N_{i}}$. The definitions of coefficients and the Levi-Civita symbol are the same as those in Table III. By properly assigning $(i, j, k, l)$, one can obtain all legitimate TQFT actions for $G=\prod_{i=1}^{4} \mathbb{Z}_{N_{i}}$. First row: Since no $A A B$ terms in the TQFT action, no assignment for $(i, j, k, l)$ is needed.Second row: For actions with $\left(A^{i} A^{j} B^{l}+A^{i} A^{k} B^{l}+A^{j} A^{k} B^{l}\right)$ terms, the assignments for $(i, j, k, l)$ are $(1,2,3,4),(2,3,4,1),(3,4,1,2)$, and $(4,1,2,3)$. For example, by taking $(i, j, k, l)=(4,1,2,3)$, we reobtain the second TQFT action in Table III.Third row: For actions with $\left(A^{i} A^{j} B^{k}+A^{i} A^{j} B^{l}\right)$ terms, there are six assignments for $(i, j, k, l):(3,4,1,2),(2,4,1,3),(2,3,1,4),(4,1,2,3),(3,1,2,4)$, and $(1,2,3,4)$. By taking $(i, j, k, l)=(1,2,3,4)$, we reobtain the third TQFT action in Table III.

\begin{tabular}{|c|c|c|}
\hline Compatible braiding phases & TQFT actions & Gauge transformations \\
\hline $\begin{array}{l}\Theta_{r}^{\mathrm{H}}=\frac{2 \pi}{N_{r}} \\
\Theta_{s, s \mid r}^{3 \mathrm{~L}}=-2 \cdot \Theta_{r, s \mid s}^{3 \mathrm{~L}}=\frac{4 \pi q_{r s s}}{N_{r} N_{s}} \\
\Theta_{r, r \mid s}^{3 \mathrm{~L}}=-2 \cdot \Theta_{s, r \mid r}^{3 \mathrm{~L}}=\frac{4 \pi s_{s r r}}{N_{s} N_{r}} \\
\Theta_{s, t \mid r}^{3 \mathrm{~L}}=-\Theta_{r, t \mid s}^{3 \mathrm{~L}}=\frac{2 \pi q_{r s t}}{N_{r} N_{s}} \\
\Theta_{t, r \mid s}^{3 \mathrm{~L}}=-\Theta_{s, r \mid t}^{3 \mathrm{~L}}=\frac{2 \pi q_{s t r}}{N_{s} N_{t}} \\
\Theta_{1,2,3,4}^{4 \mathrm{~L}}=\frac{2 \pi q_{1234}}{N_{1} N_{2} N_{3} N_{4}}\end{array}$ & $\begin{array}{l}\int \sum_{r=1}^{4} \frac{N_{r}}{2 \pi} B^{r} d A^{r} \\
\quad+\sum_{r<s}\left[\frac{q_{r s s}}{(2 \pi)^{2}} A^{r} A^{s} d A^{s}+\frac{q_{s r r}}{(2 \pi)^{2}} A^{s} A^{r} d A^{r}\right] \\
\quad+\sum_{r<s<t}\left[\frac{q_{r s t}}{(2 \pi)^{2}} A^{r} A^{s} d A^{t}+\frac{q_{s t r}}{(2 \pi)^{2}} A^{s} A^{t} d A^{r}\right] \\
\quad+\frac{q_{1234}}{(2 \pi)^{3}} A^{1} A^{2} A^{3} A^{4}\end{array}$ & $\begin{aligned} A^{r} & \rightarrow A^{r}+d \chi^{r} \\
B^{r} & \rightarrow B^{r}+d V^{r} \\
& +\sum_{s}\left(\frac{q_{r s s}}{2 \pi N_{r}} d \chi^{s} A^{s}-\frac{q_{s r r}}{2 \pi N_{r}} d \chi^{s} A^{r}\right) \\
& +\sum_{a<b<c} \frac{q_{a b c}}{2 \pi N_{r}}\left(\delta_{r, a} d \chi^{b} A^{c}-\delta_{r, b} d \chi^{a} A^{c}\right) \\
& +\sum_{a<b<c} \frac{q_{b a c}}{2 \pi N_{r}}\left(\delta_{r, b} d \chi^{c} A^{b}-\delta_{r, c} d \chi^{b} A^{a}\right) \\
& -\frac{1}{2} \sum_{s, t, u} \frac{q_{1234}}{(2 \pi)^{2} N_{r}} \epsilon^{r s t u} A^{s} A^{t} \chi^{u} \\
& +\frac{1}{2} \sum_{s, t, u} \frac{q_{1234}}{(2 \pi)^{2} N_{r}} \epsilon^{r s t u} A^{s} \chi^{t} d \chi^{u} \\
& +\frac{1}{6} \sum_{s, t, u} \frac{q_{1234}}{(2 \pi)^{2} N_{r}} \epsilon^{r s t u} \chi^{s} d \chi^{t} d \chi^{u}\end{aligned}$ \\
\hline $\begin{array}{l}\Theta_{r}^{\mathrm{H}}=\frac{2 \pi}{N_{r}} \\
\Theta_{s, s \mid r}^{3 \mathrm{~L}}=-2 \cdot \Theta_{r, s \mid s}^{3 \mathrm{~L}}=\frac{4 \pi q_{r s s}}{N_{r} N_{s}} \\
\Theta_{j, k \mid i}^{3 \mathrm{~L}}=-\Theta_{i, k \mid j}^{3 \mathrm{~L}}=\frac{2 \pi q_{i j k}}{N_{i} N_{j}} \\
\Theta_{k, i \mid j}^{3 \mathrm{~L}}=-\Theta_{j, i \mid k}^{3 \mathrm{~L}}=\frac{2 \pi q_{j k i}}{N_{j} N_{k}} \\
\Theta_{i, j \mid l}^{\mathrm{BR}}=\frac{2 \pi p_{i j, l}}{N_{i} N_{j} N_{l}} \\
\Theta_{i, k \mid l}^{\mathrm{BR}}=\frac{2 \pi p_{i k, l}}{N_{i} N_{k} N_{l}} \\
\Theta_{j, k \mid l}^{\mathrm{BR}}=\frac{2 \pi p_{j k, l}}{N_{j} N_{k} N_{l}}\end{array}$ & $\begin{array}{l}\sum_{r=1}^{4} \frac{N_{r}}{2 \pi} B^{r} d A^{r} \\
+\sum_{r \neq l, s \neq l} \frac{q_{r s s}}{(2 \pi)^{2}} A^{r} A^{s} d A^{s} \\
+\frac{q_{i j k}}{(2 \pi)^{2}} A^{i} A^{j} d A^{k}+\frac{q_{j k i}}{(2 \pi)^{2}} A^{j} A^{k} d A^{i} \\
+\frac{p_{i j, l}}{(2 \pi)^{2}} A^{i} A^{j} B^{l} \\
+\frac{p_{i k, l}}{(2 \pi)^{2}} A^{i} A^{k} B^{l} \\
+\frac{p_{j k, l}}{(2 \pi)^{2}} A^{j} A^{k} B^{l}\end{array}$ & $\begin{aligned} A^{r} & \rightarrow A^{r}+d \chi^{r} \\
& -\sum_{a, b} \frac{p_{a b, l}}{2 \pi N_{4}} \delta_{r, l}\left(\chi^{a} A^{b}+\frac{1}{2} \chi^{a} d \chi^{b}\right) \\
B^{r} & \rightarrow B^{r}+d V^{r} \\
& +\sum_{r \neq l, s \neq l}\left[\frac{q_{r s s}}{2 \pi N_{r}} d \chi^{s} A^{s}-\frac{q_{s r r}}{2 \pi N_{r}} d \chi^{s} A^{r}\right] \\
& +\sum_{r \neq l, s \neq l}\left(\frac{q_{i j k}}{2 \pi N_{r}} \epsilon^{r s k} d \chi^{s} A^{k}+\frac{q_{j k i}}{2 \pi N_{r}} \epsilon^{r s i} d \chi^{s} A^{i}\right) \\
& -\sum_{r \neq l, j \neq l} \frac{p_{r s, l}}{2 \pi N_{r}}\left(\chi^{s} B^{l}-A^{s} V^{l}+\chi^{s} d V^{l}\right)\end{aligned}$ \\
\hline $\begin{array}{l}\Theta_{r}^{\mathrm{H}}=\frac{2 \pi}{N_{r}} \\
\Theta_{j, j \mid i}^{3 \mathrm{~L}}=-2 \cdot \Theta_{i, j \mid j}^{3 \mathrm{~L}}=\frac{4 \pi q_{i j j}}{N_{i} N_{j}} \\
\Theta_{i, i \mid j}^{3 \mathrm{~L}}=-2 \cdot \Theta_{j, i \mid i}^{3 \mathrm{~L}}=\frac{4 \pi q_{j i i}}{N_{j} N_{i}} \\
\Theta_{i, j \mid k}^{\mathrm{BR}}=\frac{2 \pi p_{i j, k}}{N_{i} N_{j} N_{k}} \\
\Theta_{i, j \mid l}^{\mathrm{BR}}=\frac{2 \pi p_{i j, l}}{N_{i} N_{j} N_{l}}\end{array}$ & $\begin{aligned} \int & \sum_{r=1}^{4} \frac{N_{r}}{2 \pi} B^{r} d A^{r} \\
& +\frac{q_{i j j}}{(2 \pi)^{2}} A^{i} A^{j} d A^{j}+\frac{q_{j i i}}{(2 \pi)^{2}} A^{j} A^{i} d A^{i} \\
& +\frac{p_{i j, k}}{(2 \pi)^{2}} A^{i} A^{j} B^{k}+\frac{p_{i j, l}}{(2 \pi)^{2}} A^{i} A^{j} B^{l}\end{aligned}$ & $\begin{aligned} A^{r} & \rightarrow A^{r}+d \chi^{r} \\
& -\left(\frac{p_{i j, r}}{2 \pi N_{r}} \delta_{r, k}+\frac{p_{i j, r}}{2 \pi N_{r}} \delta_{r, l}\right)\left(\chi^{i} A^{j}+\frac{1}{2} \chi^{i} d \chi^{j}\right) \\
& +\left(\frac{p_{i j, r}}{2 \pi N_{r}} \delta_{r, k}+\frac{p_{i j, r}}{2 \pi N_{r}} \delta_{r, l}\right)\left(\chi^{j} A^{i}+\frac{1}{2} \chi^{j} d \chi^{i}\right) \\
B^{r} & \rightarrow B^{r}+d V^{r} \\
& +\sum_{s \neq k, l}\left(\frac{q_{r s s}}{2 \pi N_{r}} d \chi^{s} A^{s}-\frac{q_{j r r}}{2 \pi N_{r}} d \chi^{s} A^{r}\right) \\
& -\sum_{s} \frac{p_{i j, s}}{2 \pi N_{r}} \delta_{r, i}\left(\chi^{j} B^{s}-A^{j} V^{s}+\chi^{j} d V^{s}\right) \\
& +\sum_{s} \frac{p_{i j, s}}{2 \pi N_{r}} \delta_{r, j}\left(\chi^{i} B^{s}-A^{i} V^{s}+\chi^{i} d V^{s}\right)\end{aligned}$ \\
\hline
\end{tabular}

where $Y^{i}$ is a shift term with $\oint Y^{i} \notin \frac{2 \pi}{N_{i}} \mathbb{Z}_{N_{i}}$ hence the $\mathbb{Z}_{N_{2}}$ cyclic group structure is encoded in $\oint A^{2} \in \frac{2 \pi}{N_{2}} \mathbb{Z}_{N_{2}}$. Under the gauge transformations Eqs. (41), the variation of action Eq. (40) (boundary terms are neglected) is

$$
\begin{aligned}
\Delta S= & \int \frac{N_{1}}{2 \pi} Y^{1} d A^{1}+\frac{N_{2}}{2 \pi} Y^{2} d A^{2} \\
& +\frac{p}{(2 \pi)^{2}}\left[\left(d \chi^{1} A^{2} B^{2}+A^{1} d \chi^{2} B^{2}+d \chi^{1} d \chi^{2} B^{2}\right)\right. \\
& +\left(A^{1} A^{2} d V^{2}+d \chi^{1} A^{2} d V^{2}+A^{1} d \chi^{2} d V^{2}\right) \\
& \left.+\left(A^{1} A^{2} Y^{2}+d \chi^{1} A^{2} Y^{2}+A^{1} d \chi^{2} Y^{2}+d \chi^{1} d \chi^{2} Y^{2}\right)\right]
\end{aligned}
$$

which should be an integral of total derivative terms in order to be gauge invariant. Focus on the $d \chi^{1} d \chi^{2} B^{2}$ term which is not a total derivative term: we want to eliminate it by subtraction or by absorbing it into a total derivative term. If we want to eliminate $d \chi^{1} d \chi^{2} B^{2}$ by subtraction, the only way is to require

$$
\begin{gathered}
\frac{p}{(2 \pi)^{2}} d \chi^{1} d \chi^{2} B^{2}+\frac{p}{(2 \pi)^{2}} d \chi^{1} d \chi^{2} Y^{2} \\
=\frac{p}{(2 \pi)^{2}} d \chi^{1} d \chi^{2}\left(B^{2}-B^{2}+\cdots\right),
\end{gathered}
$$

since $d \chi^{1} d \chi^{2} Y^{2}$ is the only term containing $d \chi^{1} d \chi^{2}$ in Eq. (42). However, Eq. (43) means that $Y^{2}=-B^{2}+\cdots$, thus the gauge transformation of $B^{2}$ is

$$
B^{2} \rightarrow d V^{2}+\cdots
$$

which is not a proper gauge transformation for $B^{2}$. If we want to absorb $d \chi^{1} d \chi^{2} B^{2}$ to a total derivative term, we could make 
use of

$$
d\left(\chi^{1} d \chi^{2} B^{2}\right)=d \chi^{1} d \chi^{2} B^{2}-\chi^{1} d \chi^{2} d B^{2} .
$$

However, this attempt fails since there is no $-\chi^{1} d \chi^{2} d B^{2}$ term in $\Delta S$. Therefore, Eqs. (41) are not the proper gauge transformations for $S$.

Next, we assume that gauge transformations are

$$
\begin{aligned}
& A^{1} \rightarrow A^{1}+d \chi^{1}, \\
& A^{2} \rightarrow A^{2}+d \chi^{2}+X^{2}, \\
& B^{1} \rightarrow B^{1}+d V^{1}+Y^{1}, \\
& B^{2} \rightarrow B^{2}+d V^{2},
\end{aligned}
$$

where $X^{2}$ and $Y^{1}$ are shift terms with $\oint X^{2} \notin \frac{2 \pi}{N_{2}} \mathbb{Z}_{N_{2}}$ and $\oint Y^{1} \notin \frac{2 \pi}{N_{1}} \mathbb{Z}_{N_{1}}$. The $\mathbb{Z}_{N_{2}}$ cyclic group structure is encoded in $\oint B^{2} \in \frac{2 \pi}{N_{2}} \mathbb{Z}_{N_{2}}$. Under (46), the variation of action (40) is

$$
\begin{aligned}
\Delta S= & \int \frac{N_{1}}{2 \pi} Y^{1} d A^{1}+\frac{N_{2}}{2 \pi} B^{2} d X^{2} \\
& +\frac{p}{(2 \pi)^{2}}\left(d \chi^{1} A^{2} B^{2}+A^{1} d \chi^{2} B^{2}+d \chi^{1} d \chi^{2} B^{2}\right) \\
& +\frac{p}{(2 \pi)^{2}}\left(A^{1} X^{2} B^{2}+d \chi^{1} X^{2} B^{2}\right) \\
& +\frac{p}{(2 \pi)^{2}}\left(A^{1} A^{2} d V^{2}+d \chi^{1} A^{2} d V^{2}+A^{1} d \chi^{2} d V^{2}\right) \\
& +\frac{p}{(2 \pi)^{2}}\left(A^{1} X^{2} d V^{2}+d \chi^{1} X^{2} d V^{2}\right)
\end{aligned}
$$

which should be an integral of total derivative terms. Focus on the $d \chi^{1} A^{2} d V^{2}$ term: we want to eliminate it by subtraction or by absorbing it into a total derivative term. If we want to eliminate $d \chi^{1} A^{2} d V^{2}$ by subtraction, the only way is to require,

$$
\begin{gathered}
\frac{p}{(2 \pi)^{2}} d \chi^{1} A^{2} d V^{2}+\frac{p}{(2 \pi)^{2}} d \chi^{1} X^{2} d V^{2} \\
=\frac{p}{(2 \pi)^{2}} d \chi^{1}\left(A^{2}-A^{2}+\cdots\right) d V^{2},
\end{gathered}
$$

which means that $X^{2}=-A^{2}+\cdots$ hence

$$
A^{2} \rightarrow d \chi^{2}+\cdots,
$$

an ill-defined gauge transformation for $A^{2}$. If we want to absorb $d \chi^{1} A^{2} d V^{2}$ into a total derivative term, we need a $\chi^{1} d A^{2} d V^{2}$ term since

$$
d\left(\chi^{1} A^{2} d V^{2}\right)=d \chi^{1} A^{2} d V^{2}+\chi^{1} d A^{2} d V^{2},
$$

but there is no a $\chi^{1} d A^{2} d V^{2}$ term in $\Delta S$, so this attempt fails. Therefore, Eq. (46) is not the proper gauge transformations for the action (40) either.

Since neither (41) nor (46) serves as proper gauge transformations, it is impossible to construct gauge transformations respecting $\mathbb{Z}_{N_{2}}$ cyclic group structure for the action (40). This means that the action (40) is not a legitimate TQFT action. Therefore, $\Theta_{1,2 \mid 2}^{\mathrm{BR}}$ is not even a well-defined braiding phase, not to mention its compatibility with other braiding phases. In other words, when $G=\prod_{i=1}^{2} \mathbb{Z}_{N_{i}}$, BR braidings cannot be realized.

\section{B. Incompatibility: $\Theta_{1,2 \mid 3}^{\mathrm{BR}}$ and $\Theta_{2,3 \mid 1}^{\mathrm{BR}}$}

In previous sections, we have known that the $A^{1} A^{2} B^{3}$ term describes the BR braiding with a phase denoted as $\Theta_{1,2 \mid 3}^{\mathrm{BR}}$. One may expect that, in the same system, there could exist another BR braiding process described by an $A^{2} A^{3} B^{1}$ term and its braiding phase $\Theta_{2,3 \mid 1}^{\mathrm{BR}}$. Assuming that these two BR braidings could be supported in the same system, the TQFT action should be

$$
S=\int \sum_{i=1}^{3} \frac{N_{i}}{2 \pi} B^{i} d A^{i}+\frac{p_{12,3}}{(2 \pi)^{2}} A^{1} A^{2} B^{3}+\frac{p_{23,1}}{(2 \pi)^{2}} A^{2} A^{3} B^{1} .
$$

In the action Eq. (51), $B^{2}$ is a Lagrange multiplier imposing $d A^{2}=0$, i.e., $A^{2} \rightarrow A^{2}+d \chi^{2}$. Therefore the $\mathbb{Z}_{N_{2}}$ cyclic group structure is encoded in $\oint A^{2} \in \frac{2 \pi}{N_{2}} \mathbb{Z}_{N_{2}}$. To preserve the $\mathbb{Z}_{N_{1}}$ and $\mathbb{Z}_{N_{3}}$ cyclic group structure, at least one of $A^{i} \rightarrow$ $A^{i}+d \chi^{i}$ and $B^{i} \rightarrow B^{i}+d V^{i}(i=1,3)$ is required. We find that, if the gauge transformations preserve $\mathbb{Z}_{N_{1}}$ and $\mathbb{Z}_{N_{3}}$ cyclic group structure, the action Eq. (51) would never be gauge invariant, i.e., the braiding processes it describes are gauge dependent. The reason is that no matter how we modify the gauge transformations, there are stubborn terms in $\Delta S$ which cannot be eliminated by subtraction nor be absorbed into a total derivative term, meaning $\Delta S$ could never be $0 \bmod 2 \pi$. The details of the calculation can be found in Appendix $\mathrm{C} 1$. Therefore, $A^{1} A^{2} B^{3}$ and is incompatible with $A^{2} A^{3} B^{1}$, i.e., $\Theta_{2,3 \mid 1}^{\mathrm{BR}}$ is incompatible with $\Theta_{1,2 \mid 3}^{\mathrm{BR}}$.

In the same manner, we can derive that any two of $\left(\Theta_{2,3 \mid 1}^{\mathrm{BR}}, \Theta_{3,1 \mid 2}^{\mathrm{BR}}, \Theta_{1,2 \mid 3}^{\mathrm{BR}}\right)$ are mutually incompatible.

\section{Incompatibility: $\Theta_{3,3 \mid 1}^{3 \mathrm{~L}}$ and $\Theta_{1,2 \mid 3}^{\mathrm{BR}}$}

In this and following subsections, we investigate the incompatibility between three-loop braidings and BR braidings.

As the first example, we consider these two braiding processes: a three-loop braiding with phase $\Theta_{3,3 \mid 1}^{3 \mathrm{~L}}$, corresponding to an $A^{1} A^{3} d A^{3}$ term, and a BR braiding with phase $\Theta_{1,2 \mid 3}^{\mathrm{BR}}$, corresponding to an $A^{1} A^{2} B^{3}$ term. If theses two braiding processes are compatible, the total TQFT action should $b$

$$
S=\int \sum_{i=1}^{3} \frac{N_{i}}{2 \pi} B^{i} d A^{i}+\frac{q_{133}}{(2 \pi)^{2}} A^{1} A^{3} d A^{3}+\frac{p_{12,3}}{(2 \pi)^{2}} A^{1} A^{2} B^{3} .
$$

In the action Eq. (52), $B^{1}$ and $B^{2}$ serve as Lagrange multipliers, imposing $d A^{1}=d A^{2}=0$, i.e., $A^{1,2} \rightarrow A^{1,2}+d g^{1,2}$. The $\mathbb{Z}_{N_{1}}$ and $\mathbb{Z}_{N_{2}}$ cyclic group structures are encoded in $\oint A^{1} \in$ $\frac{2 \pi}{N_{1}} \mathbb{Z}_{N_{1}}$ and $\oint A^{2} \in \frac{2 \pi}{N_{2}} \mathbb{Z}_{N_{2}}$, respectively. For the $\mathbb{Z}_{N_{3}}$ cyclic group structure, we have two choices: we can encoded it in $\oint A^{3} \in \frac{2 \pi}{N_{3}} \mathbb{Z}_{N_{3}}$, corresponding to gauge transformations

$$
\begin{aligned}
& A^{i} \rightarrow A^{i}+d \chi^{i}, \\
& B^{i} \rightarrow B^{i}+d V^{i}+Y^{i} ;
\end{aligned}
$$

or $\oint B^{3} \in \frac{2 \pi}{N_{3}} \mathbb{Z}_{N_{3}}$, corresponding to gauge transformations

$$
\begin{aligned}
A^{1,2} & \rightarrow A^{1,2}+d \chi^{1,2}, \\
A^{3} & \rightarrow A^{3}+d \chi^{3}+X^{3}, \\
B^{1,2} & \rightarrow B^{1,2}+d V^{1,2}+Y^{1,2}, \\
B^{3} & \rightarrow B^{3}+d V^{3} .
\end{aligned}
$$


However, similar to the case in Sec. IV A and Sec. IV B, neither Eqs. (53) nor Eqs. (54) could make the action Eq. (52) gauge invariant up to boundary terms, due to the existence of stubborn terms. Details of derivations can be found in Appendix C2. If the action is forced to be gauge invariant up to boundary terms, the $\mathbb{Z}_{N_{3}}$ cyclic group structure would be violated. This dilemma reveals that Eq. (52) is not a legitimate TQFT action. So we conclude that $A^{1} A^{3} d A^{3}$ is incompatible with $A^{1} A^{2} B^{3}$, i.e., $\Theta_{1,3 \mid 3}^{3 \mathrm{~L}}$ is incompatible with $\Theta_{1,2 \mid 3}^{\mathrm{BR}}$.

\section{Incompatibility: $\Theta_{2,3 \mid 1}^{3 \mathrm{~L}}$ and $\Theta_{1,2 \mid 3}^{\mathrm{BR}}$}

In this subsection, we consider these two braiding processes: a three-loop braiding corresponding to $\Theta_{2,3 \mid 1}^{3 \mathrm{~L}}$ and an $A^{1} A^{2} d A^{3}$ term, and a BR braiding corresponding to $\Theta_{1,2 \mid 3}^{\mathrm{BR}}$ and an $A^{1} A^{2} B^{3}$ term. If $\Theta_{2,3 \mid 1}^{3 \mathrm{~L}}$ is compatible with $\Theta_{1,2 \mid 3}^{\mathrm{BR}}$, the total TQFT action should be

$$
S=\int \sum_{i=1}^{3} \frac{N_{i}}{2 \pi} B^{i} d A^{i}+\frac{q_{123}}{(2 \pi)^{2}} A^{1} A^{2} d A^{3}+\frac{p_{12,3}}{(2 \pi)^{2}} A^{1} A^{2} B^{3},
$$

where the coefficients are $q_{123}=\frac{k_{1} N_{1} N_{2}}{N_{12}}, k_{1} \in \mathbb{Z}_{N_{123}}$ and $p_{12,3}=\frac{k_{2} N_{1} N_{2} N_{3}}{N_{123}}, k_{2} \in \mathbb{Z}_{N_{123}}$, determined by the large gauge invariance. In Eq. (55), $B^{1}$ and $B^{2}$ serve as Lagrange multipliers, imposing $d A^{1}=d A^{2}=0$, i.e., $A^{1,2} \rightarrow A^{1,2}+d \chi^{1,2}$. We can find a set of gauge transformations that seems to respect both $\mathbb{Z}_{N_{3}}$ cyclic group structure and gauge invariance (see Appendix C 3 for derivation):

$$
\begin{aligned}
& A^{1} \rightarrow A^{1}+d \chi^{1}, \\
& A^{2} \rightarrow A^{2}+d \chi^{2}, \\
& A^{3} \rightarrow A^{3}+d \chi^{3}+X^{3}, \\
& B^{1} \rightarrow B^{1}+d V^{1}+Y^{1}, \\
& B^{2} \rightarrow B^{2}+d V^{2}+Y^{2}, \\
& B^{3} \rightarrow B^{3}+d V^{3},
\end{aligned}
$$

where

$$
\begin{aligned}
X^{3}= & -\frac{p_{12,3}}{(2 \pi) N_{3}}\left(\chi^{1} A^{2}+\frac{1}{2} \chi^{1} d \chi^{2}\right)+\frac{p_{12,3}}{(2 \pi) N_{3}}\left(\chi^{2} A^{1}+\frac{1}{2} \chi^{2} d \chi^{1}\right), \\
Y^{1}= & \frac{q_{123}}{2 \pi N_{1}} d \chi^{2} A^{3}-\frac{p}{(2 \pi) N_{1}}\left(\chi^{2} B^{3}-A^{2} V^{3}+\chi^{2} d V^{3}\right) \\
& +\frac{q_{123}}{(2 \pi)^{2}} \cdot \frac{p_{12,3}}{(2 \pi) N_{3}} \cdot \frac{2 \pi}{N_{1}} \cdot\left[\chi^{1} A^{2} d \chi^{2}-\left(A^{1} A^{2} \chi^{2}+A^{1} d \chi^{2} \chi^{2}+d \chi^{1} A^{2} \chi^{2}+d \chi^{1} d \chi^{2} \chi^{2}\right)\right], \\
Y^{2}= & -\frac{q_{123}}{2 \pi N_{2}} d \chi^{1} A^{3}+\frac{p_{12,3}}{(2 \pi) N_{2}}\left(\chi^{1} B^{3}-A^{1} V^{3}+\chi^{1} d V^{3}\right) \\
& +\frac{q_{123}}{(2 \pi)^{2}} \cdot \frac{p_{12,3}}{(2 \pi) N_{3}} \cdot \frac{2 \pi}{N_{2}} \cdot\left[-d\left(A^{1} \chi^{1}\right) \chi^{2}+\left(A^{1} A^{2} \chi^{1}+A^{1} d \chi^{2} \chi^{1}+d \chi^{1} A^{2} \chi^{1}+d \chi^{1} d \chi^{2} \chi^{1}\right)\right] .
\end{aligned}
$$

Nevertheless, the action Eq. (55) is still not a legitimate TQFT theory: the coefficient of $A^{1} A^{2} d A^{3}$ in Eq. (55) is actually identical to 0 . To see this, we first integrate out $A^{3}$ in $S$, i.e., sum over all possible nontrivial compactness contributions from $d A^{3}$, leading to a constraint

$$
\int \frac{q_{123}}{(2 \pi)^{2}} A^{1} A^{2}+\frac{N_{3}}{2 \pi} B^{3} \in \mathbb{Z} .
$$

Since the $\mathbb{Z}_{N_{3}}$ cyclic group structure is encoded in $\oint \frac{N_{3}}{2 \pi} B^{3} \in$ $\mathbb{Z}$, Eq. (58) requires that

$$
\frac{q_{123}}{(2 \pi)^{2}} \int A^{1} A^{2} \in \mathbb{Z}
$$

i.e., (consider a two-manifold $\mathcal{M}^{2}=S^{1} \times S^{1}$ )

$$
\begin{aligned}
\frac{q_{123}}{(2 \pi)^{2}} \int_{\mathcal{M}^{2}} A^{1} A^{2} & =\frac{k_{1} N_{1} N_{2}}{(2 \pi)^{2} N_{12}} \int_{\mathcal{S}^{1}} A^{1} \int_{\mathcal{S}^{1}} A^{2} \\
& =\frac{k_{1} N_{1} N_{2}}{(2 \pi)^{2} N_{12}} \times \frac{2 \pi m_{1}}{N_{1}} \times \frac{2 \pi m_{2}}{N_{2}} \\
& =\frac{k_{1} m_{1} m_{2}}{N_{12}} \in \mathbb{Z},
\end{aligned}
$$

where $k_{1} \in \mathbb{Z}_{N_{123}}, \quad m_{1} \in \mathbb{Z}, \quad m_{2} \in \mathbb{Z}$. Since $m_{1}$ and $m_{2}$ can be arbitrary integers, the sufficient condition for Eq. (60) is

$$
k_{1}=l N_{12}, l \in \mathbb{Z} .
$$

On the other hand, $N_{12}$ is the integral multiple of $N_{123}$, thus

$$
k_{1}=l N_{12}=l\left(n N_{123}\right)=(\ln ) N_{123},
$$

where $n=\frac{N_{12}}{N_{123}}$. Notice that $k_{1} \in \mathbb{Z}_{N_{123}}$, which means that

$$
k_{1}=(\ln ) N_{123} \simeq 0 .
$$

Therefore, the coefficient of $A^{1} A^{2} d A^{3}$ term is required to be identical to 0 :

$$
q_{123}=\frac{k_{1} N_{1} N_{2}}{N_{12}} \simeq 0 .
$$

So far, we have argued that to preserve the $\mathbb{Z}_{N_{3}}$ fusion structure with arbitrary values of $N_{1}, N_{2}$, and $N_{3}$, the coefficient of $A^{1} A^{2} d A^{3}$ term in Eq. (55) is required to be trivial. If the coefficient of $A^{1} A^{2} d A^{3}$ is nontrivial, the action Eq. (55) cannot be gauge invariant up to boundary terms while the 
$\mathbb{Z}_{N_{3}}$ cyclic group structure is preserved. The restriction on the coefficient of $A^{1} A^{2} d A^{3}$ indicates the incompatibility between $A^{1} A^{2} d A^{3}$ and $A^{1} A^{2} B^{3}$, i.e., the incompatibility between $\Theta_{1,2 \mid 3}^{3 \mathrm{~L}}$ and $\Theta_{1,2 \mid 3}^{\mathrm{BR}}$.

\section{E. Incompatibility: $\Theta_{3,1 \mid 2}^{3 L}$ and $\Theta_{1,2 \mid 3}^{B R}$}

As the last example of the incompatibility between threeloop braidings and $\mathrm{BR}$ braidings, we consider a three-loop braiding corresponding to $\Theta_{3,1 \mid 2}^{3 \mathrm{~L}}$ as well as an $A^{2} A^{3} d A^{1}$ term, and a BR braiding corresponding to $\Theta_{1,2 \mid 3}^{\mathrm{BR}}$ as well as an $A^{1} A^{2} B^{3}$ term. The total action should be

$$
S=\int \sum_{i=1}^{3} \frac{N_{i}}{2 \pi} B^{i} d A^{i}+\frac{q_{231}}{(2 \pi)^{2}} A^{2} A^{3} d A^{1}+\frac{p_{12,3}}{(2 \pi)^{2}} A^{1} A^{2} B^{3},
$$

where the coefficients are $q_{231}=\frac{k N_{2} N_{3}}{N_{23}}, k \in \mathbb{Z}_{N_{123}}$ and $p_{12,3}=$ $\frac{l N_{1} N_{2} N_{3}}{N_{123}}, l \in \mathbb{Z}_{N_{123}}$, determined by the large gauge invariance. In the action Eq. (65), $B^{1}$ and $B^{2}$ serve as Lagrange multipliers, imposing $d A^{1}=d A^{2}=0$, i.e., $A^{1,2} \rightarrow A^{1,2}+d g^{1,2}$.

If the $\mathbb{Z}_{N_{3}}$ cyclic group structure is encoded in $\oint A^{3} \in$ $\frac{2 \pi}{N_{3}} \mathbb{Z}_{N_{3}}$, i.e., $A^{3} \rightarrow A^{3}+d \chi^{3}$, we cannot find a set of gauge transformations with $A^{i} \rightarrow A^{i}+d \chi^{i}(i=1,2,3)$ under which the action Eq. (65) is invariant up to boundary terms. The reason is the same as those in Secs. IV A and IV C.

If we encode the $\mathbb{Z}_{N_{3}}$ cyclic group structure in $\oint B^{3} \in$ $\frac{2 \pi}{N_{3}} \mathbb{Z}_{N_{3}}$, i.e., $B^{3} \rightarrow B^{3}+d V^{3}$, we can find a set of gauge transformations under which Eq. (65) is invariant up to boundary terms

$$
\begin{aligned}
& A^{1} \rightarrow A^{1}+d g^{1}, \\
& A^{2} \rightarrow A^{2}+d g^{2} \\
& A^{3} \rightarrow A^{3}+d \chi^{3}+\frac{p_{12,3}}{(2 \pi) N_{3}}\left(-\chi^{1} A^{2}+A^{1} \chi^{2}-\chi^{1} d \chi^{2}\right),
\end{aligned}
$$

$$
\begin{aligned}
B^{1} \rightarrow & B^{1}+d V^{1}-\frac{p_{12,3}}{(2 \pi) N_{1}}\left(\chi^{2} B^{3}-A^{2} V^{3}+\chi^{2} d V^{3}\right) \\
& -\frac{q_{231}}{(2 \pi) N_{1}}\left(d \chi^{2} A^{3}+A^{2} d \chi^{3}+A^{2} X^{3}+d \chi^{2} X^{3}\right), \\
B^{2} \rightarrow & B^{2}+d V^{2}+\frac{p_{12,3}}{(2 \pi) N_{2}}\left(\chi^{1} B^{3}-A^{1} V^{3}+\chi^{1} d V^{3}\right), \\
B^{3} \rightarrow & B^{3}+d V^{3} .
\end{aligned}
$$

At first glance, one may think that Eqs. (66) are a proper set of gauge transformations for Eq. (65); thus the action Eq. (65) is a legitimate TQFT action. However, we argue that this is not true. We provide the following two arguments.

In the first argument, we consider the gauge transformations at limiting cases. Let us set $p_{12,3}=0$, i.e., turn off the $A^{1} A^{2} B^{3}$ term, the action Eq. (65) becomes

$$
S=\int \sum_{i=1}^{3} \frac{N_{i}}{2 \pi} B^{i} d A^{i}+\frac{q_{231}}{(2 \pi)^{2}} A^{2} A^{3} d A^{1}
$$

and the corresponding gauge transformations should be

$$
\begin{aligned}
& A^{i} \rightarrow A^{i}+d \chi^{i}, \\
& B^{1} \rightarrow B^{1}+d V^{1}, \\
& B^{2} \rightarrow B^{2}+d V^{2}+\frac{q_{231}}{2 \pi N_{2}} d \chi^{3} A^{1}, \\
& B^{3} \rightarrow B^{3}+d V^{3}-\frac{q_{231}}{2 \pi N_{3}} d \chi^{2} A^{1} .
\end{aligned}
$$

According to Ref. [34], gauge transformations Eqs. (68) is motivated by a microscopic derivation of the action Eq. (67). However, if we set $p_{12,3}=0$ in Eqs. (66), we cannot correctly recover the gauge transformations to Eqs. (68). Therefore, we claim that Eqs. (66) are not proper gauge transformations for the action Eq. (65).

In fact, if we expect that by setting $q_{231}=0$ or $p_{12,3}=0$ the gauge transformations for the action Eq. (65) would recover to Eq. (15) or Eq. (68), the gauge transformations for the action Eq. (65) should take the form of

$$
\begin{aligned}
& A^{1} \rightarrow A^{1}+d \chi^{1}, \\
& A^{2} \rightarrow A^{2}+d \chi^{2}, \\
& A^{3} \rightarrow A^{3}+d \chi^{3}+\frac{p_{12,3}}{(2 \pi)^{2}} \frac{2 \pi}{N_{3}}\left(-\chi^{1} A^{2}+A^{1} \chi^{2}-\chi^{1} d \chi^{2}\right)+f_{A}^{3}\left(p_{12,3}, q_{231}\right) \\
& B^{1} \rightarrow B^{1}+d V^{1}-\frac{p_{12,3}}{(2 \pi) N_{1}}\left(\chi^{2} B^{3}-A^{2} V^{3}+\chi^{2} d V^{3}\right)+f_{B}^{1}\left(p_{12,3}, q_{231}\right) \\
& B^{2} \rightarrow B^{2}+d V^{2}+\frac{q_{231}}{2 \pi N_{2}} d \chi^{3} A^{1}+\frac{p_{12,3}}{(2 \pi) N_{2}}\left(\chi^{1} B^{3}-A^{1} V^{3}+\chi^{1} d V^{3}\right)+f_{B}^{2}\left(p_{12,3}, q_{231}\right) \\
& B^{3} \rightarrow B^{3}+d V^{3}-\frac{q_{231}}{2 \pi N_{3}} d \chi^{2} A^{1}+f_{B}^{3}\left(p_{12,3}, q_{231}\right),
\end{aligned}
$$

where the gauge parameters $f_{A}^{3}\left(p_{12,3}, q_{231}\right)$ and $f_{B}^{i}\left(p_{12,3}, q_{231}\right)$ with $i=1,2,3$ are identical to 0 once $p_{12,3}=0$ or $q_{231}=0$. We can see that the $\mathbb{Z}_{N_{3}}$ cyclic group structure is no longer preserved under gauge transformation Eqs. (69).

The second argument exploits the identity

$$
A^{2} A^{3} d A^{1}=d\left(A^{1} A^{2} A^{3}\right)-A^{3} A^{1} d A^{2}-A^{1} A^{2} d A^{3} .
$$

Using Eq. (70) we can rewrite the action Eq. (65) as

$$
\begin{aligned}
S= & \int \sum_{i=1}^{3} \frac{N_{i}}{2 \pi} B^{i} d A^{i}+\frac{p_{12,3}}{(2 \pi)^{2}} A^{1} A^{2} B^{3} \\
& +\frac{q_{231}}{(2 \pi)^{2}}\left[d\left(A^{1} A^{2} A^{3}\right)-A^{3} A^{1} d A^{2}-A^{1} A^{2} d A^{3}\right] .
\end{aligned}
$$


Similar to the case in Sec. IV D, we can integrate out $A^{3}$ and obtain a constraint

$$
\int \frac{N_{3}}{2 \pi} B^{3}-\frac{q_{231}}{(2 \pi)^{2}} A^{1} A^{2} \in \mathbb{Z} .
$$

Since in the gauge transformations Eqs. (56) the $\mathbb{Z}_{N_{3}}$ cyclic group structure is encoded in $\oint B^{3}=\frac{2 \pi}{N_{3}} \mathbb{Z}_{N_{3}}$, we require that

$$
\int \frac{q_{231}}{(2 \pi)^{2}} A^{1} A^{2} \in \mathbb{Z} \text {. }
$$

In other words, we require that (consider $\mathcal{M}^{2}=S^{1} \times S^{1}$ )

$$
\begin{aligned}
\frac{q_{231}}{(2 \pi)^{2}} \int_{\mathcal{M}^{2}} A^{1} A^{2} & =\frac{k N_{2} N_{3}}{(2 \pi)^{2} N_{23}} \int_{\mathcal{S}^{1}} A^{1} \int_{\mathcal{S}^{1}} A^{2} \\
& =\frac{k N_{2} N_{3}}{(2 \pi)^{2} N_{23}} \cdot \frac{2 \pi m_{1}}{N_{1}} \cdot \frac{2 \pi m_{2}}{N_{2}} \\
& =\frac{k N_{3} m_{1} m_{2}}{N_{1} N_{23}} \in \mathbb{Z},
\end{aligned}
$$

where $k \in \mathbb{Z}_{N_{123}}$. Since $m_{1}$ and $m_{2}$ can be arbitrary integers, we need

$$
\frac{k N_{3}}{N_{1} N_{23}} \in \mathbb{Z} .
$$

Since $\frac{N_{3}}{N_{23}} \in \mathbb{Z}$, the sufficient condition for Eq. (74) to hold is

$$
k=m N_{1}, m \in \mathbb{Z} .
$$

Notice that $\frac{N_{1}}{N_{123}} \in \mathbb{Z}$ and $k \in \mathbb{Z}_{N_{123}}$, therefore

$$
k=m N_{1}=m n N_{123} \simeq 0,
$$

where $n=\frac{N_{1}}{N_{123}} \in \mathbb{Z}$. We see that the coefficient of $A^{2} A^{3} d A^{1}$ is required to be identical to 0 ,

$$
q_{231}=\frac{k N_{2} N_{3}}{N_{23}} \simeq 0,
$$

to ensure that the $\mathbb{Z}_{N_{3}}$ cyclic group structure is preserved no matter what the values of $N_{1}, N_{2}$, and $N_{3}$ are. This restriction on the coefficient of $A^{2} A^{3} d A^{1}$ reveals that $A^{2} A^{3} d A^{1}$ is incompatible with $A^{1} A^{2} B^{3}$, hence $\Theta_{31,2}^{3 \mathrm{~L}}$ and $\Theta_{12,3}^{\mathrm{BR}}$ are mutually incompatible.

\section{F. Incompatibility: $\Theta_{1,2,3,4}^{4 \mathrm{~L}}$ and $\Theta_{1,2 \mid 4}^{\mathrm{BR}}$}

Since we have demonstrated the incompatibility between BR braidings and three-loop braidings, one may wonder whether BR braiding is compatible with four-loop braiding or not. The conclusion is that, when $G=\prod_{i=1}^{4} \mathbb{Z}_{N_{i}}$, no BR braiding is compatible with four-loop braiding, i.e., each $\Theta_{i, j \mid k}^{\mathrm{BR}}$ is incompatible with $\Theta_{1,2,3,4}^{4 \mathrm{~L}}$. The proof of this claim, which follows the same idea discussed in previous sections, is detailed in Appendix C 4. Notice that, if $G=\prod_{i=1}^{5} \mathbb{Z}_{N_{i}}, \Theta_{1,2,3,4}^{4 \mathrm{~L}}$ is compatible with $\Theta_{i, j \mid 5}^{\mathrm{BR}}$, as long as $i \neq 5, j \neq 5$ and $i \neq j$. Such compatibility due to an extra gauge subgroup can be checked by applying the criteria for legitimacy of TQFT actions.

\section{COMPATIBLE BRAIDINGS IN (4+1)D SPACETIME}

In the above discussions, we have obtained compatible braidings in $(3+1) \mathrm{D}$ spacetime. In the following, we concisely discuss compatible braidings in $(4+1) \mathrm{D}$ spacetime. More systematic and quantitative studies will appear in Ref. [61] separately.

\section{A. Excitations and braiding processes in (4+1)D}

We limit the gauge group to be $G=\prod_{i=1}^{n} \mathbb{Z}_{N_{i}}$ where $n$ is the total number of cyclic subgroups. In $(4+1) \mathrm{D}$ spacetime, besides particles and loops, there is a kind exotic topological excitations, dubbed membranes, which are closed 2D surface objects in the continuum limit. The membrane excitations in three-dimensional space is topologically trivial because they are impenetrable hence identified as particle excitations. However, in four-dimensional space, the interior of a membrane excitation becomes accessible due to the extra dimension. Therefore, nontrivial braiding processes involving particles, loops, and membranes are possible in $(4+1) \mathrm{D}$ spacetime.

In field theory, a $\mathbb{Z}_{N_{i}}$ gauge theory is realized by a $B F$ term in the continuum limit. In $(3+1) \mathrm{D}$ spacetime, the $\mathbb{Z}_{N_{i}}$ cyclic group structure is encoded in the $B^{i} d A^{i}$ term, the only possible $B F$ term in $(3+1) \mathrm{D}$ spacetime which corresponds to the particle-loop braiding (see Sec. II B). In $(4+1) \mathrm{D}$ spacetime, there are two kinds of $B F$ terms with distinct physical meanings: $C^{i} d A^{i}$ and $\widetilde{B}^{i} d B^{i}$, where the three-form $C^{i}$, one-form $A^{i}$, two-form $\widetilde{B}^{i}$, and two-form $B^{i}$ are, respectively, compact $\mathbb{U}(1)$ gauge fields. Therefore, we can encode the $\mathbb{Z}_{N_{i}}$ cyclic group structure either in $C^{i} d A^{i}$, corresponding to a particle-membrane braiding; or in $\widetilde{B}^{i} d B^{i}$, corresponding to a loop-loop braiding. If we consider a $\prod_{i=1}^{n} \mathbb{Z}_{N_{i}}$ topological order in $(4+1) \mathrm{D}$ spacetime, we can even encode some $\mathbb{Z}_{N_{i}}$ gauge subgroups in $C^{i} d A^{i}$ terms while the others in $\widetilde{B}^{i} d B^{i}$ terms, which would lead to a more complicated situation in which different gauge fields can couple together resulting in many nontrivial braiding processes.

Since there are three kinds of topological excitations in $(4+1) \mathrm{D}$ spacetime, we can classify the braiding processes and identify the corresponding topological terms: (1) particle-membrane braiding, $C d A$; (2) particle-membranemembrane braiding: $C A A$; (3) loop-loop braiding, $\widetilde{B} d B$; (4) loop-membrane braidings, $B B A, B A d A, A A A B$, and $A A d B$; and (5) multimembrane braidings, $A A A A A, A A A d A$ and $\operatorname{AdAdA}$.

\section{B. An overview of compatible braiding processes in (4+1)D}

Following the discussions in $(3+1) \mathrm{D}$ spacetime, we can investigate the compatibility of braiding processes in $(4+1) \mathrm{D}$ spacetime. In other words, we check the legitimacy of TQFT actions consisting of topological terms, then distinguish compatible/incompatible braiding processes in $(4+1) \mathrm{D}$ spacetime.

First, if all $\mathbb{Z}_{N_{i}}$ cyclic group structures are encoded in particle-membrane braidings, i.e., $C^{i} d A^{i}$ terms, the compatible braiding processes are multimembrane braidings and particle-membrane-membrane braiding. Here are some examples. When $G=\prod_{i=1}^{5} \mathbb{Z}_{N_{i}}$, one of the legitimate TQFT actions is

$$
\begin{aligned}
S \sim & \int \sum_{i=1}^{5} \frac{N_{i}}{2 \pi} C^{i} d A^{i}+A^{1} A^{2} A^{3} A^{4} A^{5} \\
& +\sum_{i, j, k, l=1}^{5} A^{i} A^{j} A^{k} d A^{l}+\sum_{i, j, k=1}^{5} A^{i} d A^{j} d A^{k},
\end{aligned}
$$


where the quantized coefficients of topological terms are neglected. The action Eq. (79) indicates the compatibility of particle-membrane braidings and multimembrane braidings when $G=\prod_{i=1}^{5} \mathbb{Z}_{N_{i}}$. We believe that the TQFT action Eq. (79) describes braiding processes within the DijkgraafWitten model. There are also braiding processes beyond the Dijkgraaf-Witten model. Consider $G=\prod_{i=1}^{3} \mathbb{Z}_{N_{i}}$, one of the legitimate TQFT actions (coefficients neglected) is

$$
S \sim \int \sum_{i=1}^{3} \frac{N_{i}}{2 \pi} C^{i} d A^{i}+C^{3} A^{1} A^{2}
$$

as an analog of the BR braiding in $(3+1) \mathrm{D}, S_{\mathrm{BR}} \sim$ $\int \sum_{i=1}^{3} B^{i} d A^{i}+A^{1} A^{2} B^{3}$. We have known that, in $(3+1) \mathrm{D}$, a BR braiding is incompatible with some of the multiloop braidings (Secs. IV C-IV E). Similar incompatibility occurs in $(4+1)$ D. When $G=\prod_{i=1}^{3} \mathbb{Z}_{N_{i}}$, a multi-membrane braiding described by the $A^{1} A^{2} A^{3} d A^{1}$ term is incompatible with the loop-membrane-membrane braiding described by the $C^{3} A^{1} A^{2}$ term. Using the language of TQFT, we claim that a legitimate TQFT action in $(4+1) \mathrm{D}$ can only include one of the $C^{3} A^{1} A^{2}$ term and $A^{1} A^{2} A^{3} d A^{1}$ term. When $G=\prod_{i=1}^{3} \mathbb{Z}_{N_{i}}$, two of the legitimate TQFT actions are

$$
S \sim \int \sum_{i=1}^{3} \frac{N_{i}}{2 \pi} C^{i} d A^{i}+\sum_{i, j, k=1}^{2} A^{i} d A^{j} d A^{k}+C^{3} A^{1} A^{2}
$$

and

$$
S \sim \int \sum_{i=1}^{3} \frac{N_{i}}{2 \pi} C^{i} d A^{i}+\sum_{i=1}^{3} A^{1} A^{2} A^{3} d A^{i}+\sum_{i, j, k=1}^{3} A^{i} d A^{j} d A^{k} .
$$

Next, we consider the case in which all $\mathbb{Z}_{N_{i}}$ cyclic group structures are encoded in $\widetilde{B}^{i} d B^{i}$ terms, i.e., loop-loop braidings. In this case, the only topological excitations are loops, thus only loop-loop braidings can be supported. We can simply write down the legitimate TQFT action:

$$
S=\int \sum_{i=1}^{n} \frac{N_{i}}{2 \pi} \widetilde{B}^{i} d B^{i}
$$

Last, we tackle the more complicated yet more interesting situation: some $\mathbb{Z}_{N}$ cyclic group structures (e.g., $\mathbb{Z}_{N_{i}}$ gauge subgroups) are encoded in $\frac{N_{i}}{2 \pi} C^{i} d A^{i}$ terms while the others (e.g., $\mathbb{Z}_{K_{i}}$ gauge subgroups) in $\frac{K_{i}}{2 \pi} \widetilde{B}^{i} d B^{i}$ terms. Different assignments for $N_{i}$ 's and $K_{i}$ 's correspond to different topological orders in $(4+1) \mathrm{D}$. In this case, there are particle, loop, and membrane excitations in the system. All nontrivial braiding processes in $(4+1) \mathrm{D}$ should be taken into consideration. In the following, we will demonstrate several examples of legitimate TQFT actions from which we can directly read out compatible braiding processes in $(4+1) \mathrm{D}$.

Consider a $\prod_{i=1}^{3} \mathbb{Z}_{N_{i}}$ case in which the $\mathbb{Z}_{N_{1}}$ and $\mathbb{Z}_{N_{2}}$ cyclic group structures are encoded in $C d A$ while the $\mathbb{Z}_{K_{1}}$ cyclic group structure in $\widetilde{B} d B$, i.e., the gauge group is $G=\mathbb{Z}_{N_{1}} \times$
$\mathbb{Z}_{N_{2}} \times \mathbb{Z}_{K_{1}}$, the legitimate TQFT actions is

$$
\begin{aligned}
S \sim & \int \frac{N_{1}}{2 \pi} C^{1} d A^{1}+\frac{N_{2}}{2 \pi} C^{2} d A^{2}+\frac{K_{1}}{2 \pi} \widetilde{B}^{1} d B^{1}+\sum_{i, j, k=1}^{2} A^{i} d A^{j} d A^{k} \\
& +\sum_{i=1}^{2} B^{1} B^{1} A^{i}+\sum_{j, k=1}^{2} B^{1} A^{j} d A^{k}+\sum_{i, j=1}^{2} A^{i} A^{j} d B^{1} .
\end{aligned}
$$

Consider again a $\prod_{i=1}^{3} \mathbb{Z}_{N_{i}}$ case but this time $G=\mathbb{Z}_{N_{1}} \times$ $\mathbb{Z}_{K_{1}} \times \mathbb{Z}_{K_{2}}$, the corresponding legitimate TQFT action is

$$
\begin{aligned}
S \sim & \int \frac{N_{1}}{2 \pi} C^{1} d A^{1}+\frac{K_{1}}{2 \pi} \widetilde{B}^{1} d B^{1}+\frac{K_{2}}{2 \pi} \widetilde{B}^{2} d B^{2}+A^{1} d A^{1} d A^{1} \\
& +\sum_{i=1}^{2} B^{i} B^{j} A^{1}+\sum_{i=1}^{2} B^{i} A^{1} d A^{1} .
\end{aligned}
$$

Consider a $\prod_{i=1}^{4} \mathbb{Z}_{N_{i}}$ case in which $G=\mathbb{Z}_{N_{1}} \times \mathbb{Z}_{N_{2}} \times$ $\mathbb{Z}_{N_{3}} \times \mathbb{Z}_{K_{1}}$, the legitimate TQFT action is

$$
\begin{aligned}
S \sim & \int \sum_{i=1}^{3} \frac{N_{i}}{2 \pi} C^{i} d A^{i}+\frac{K_{1}}{2 \pi} \widetilde{B}^{1} d B^{1}+\sum_{i, j, k=1}^{3} A^{i} d A^{j} d A^{k} \\
& +C^{3} A^{1} A^{2}+\sum_{i=1}^{2} B^{1} B^{1} A^{i}+\sum_{i, j=1}^{2} B^{1} A^{i} d A^{j}
\end{aligned}
$$

Consider a $\prod_{i=1}^{5} \mathbb{Z}_{N_{i}}$ case in which $G=\mathbb{Z}_{N_{1}} \times \mathbb{Z}_{N_{2}} \times$ $\mathbb{Z}_{N_{3}} \times \mathbb{Z}_{K_{1}} \times \mathbb{Z}_{K_{2}}$, the legitimate TQFT action is

$$
\begin{aligned}
S \sim & \int \sum_{i=1}^{3} \frac{N_{i}}{2 \pi} C^{i} d A^{i}+\sum_{i=1}^{2} \frac{K_{i}}{2 \pi} \widetilde{B}^{i} d B^{i}+\sum_{i, j, k=1}^{3} A^{i} d A^{j} d A^{k} \\
& +\sum_{i, j=1}^{2} \sum_{k=1}^{3} B^{i} B^{j} A^{k}+\sum_{i=1}^{2} \sum_{j, k=1}^{2} B^{i} A^{j} d A^{k}+C^{3} A^{1} A^{2} .
\end{aligned}
$$

We should point out that the $B C$ term is also a topological term in $(4+1) \mathrm{D}$ spacetime, but we do not regard that it corresponds to any braiding process in the discussion above. Naively, because the two-form $B$ is related to the loop current $\Sigma$ via $\Sigma=\frac{1}{2 \pi} * d B$ and the three-form $C$ is related to the particle current $J$ via $J=\frac{1}{2 \pi} * d C$, one may think that $B C$ is related to the particle-loop braiding in $(4+1) \mathrm{D}$. But we argue that particle-loop braiding is actually trivial in $(4+1) \mathrm{D}$ spacetime, thus the $B C$ term does not describe the particleloop braiding. To show the triviality of particle-loop braiding in $(4+1) \mathrm{D}$ spacetime, we can first consider the particleloop braiding in $(3+1) \mathrm{D}$ spacetime in which the Hopf link formed by trajectory $\gamma_{e}$ of the particle $e$ and the loop $m$ (e.g., Fig. 1) cannot be unlinked in 3D space. However, in 4D space, due to an extra dimension, we can smoothly deform $\gamma_{e}$ such that it is unlinked with the loop $m$. This argument is analog to the fact that the nontrivial particle-particle braiding in $(2+1) \mathrm{D}$ spacetime becomes trivial in $(3+1) \mathrm{D}$ spacetime. In this manner, we claim that the particle-loop braiding in $(4+1) \mathrm{D}$ spacetime is trivial.

A question naturally arises: What is the physical effect of the $B C$ term? We noticed that there are topological terms which can generate trivial fermions from a bosonic model: $\frac{k}{4 \pi} A d A$ with an odd integer $k$ in $(2+1) \mathrm{D}$ and $B B$ in $(3+1) \mathrm{D}$. 
We conjecture that the effect of $B C$ term is similar: The emergence of trivial fermions is possible in a bosonic model described by the action $S \sim \int C d A+\widetilde{B} d B+B C$.

\section{CONCLUSION}

In this paper, when a gauge group is given, we investigate the compatibility between all three classes of root braiding processes in $(3+1) \mathrm{D}$ spacetime, i.e., particle-loop braidings, multiloop braidings, and BR braidings. We find that not all root braiding processes are mutually compatible, which is not so obvious on a superficial level. By incompatibility we mean that two braiding processes cannot be supported in the same system, i.e., there is no legitimate topological order in $(3+1)$-dimensional spacetime characterized by incompatible braiding phases. In the language of TQFT, a TQFT action describing incompatible braiding processes is not gauge invariant while preserving the cyclic group structure of each gauge subgroup. Our conclusions are drawn as follows.

When the gauge group is $G=\mathbb{Z}_{N_{1}}$, only one class of root braiding processes, i.e., particle-loop braiding, is realizable. Therefore, there is no incompatibility when $G=\mathbb{Z}_{N_{1}}$. The set of compatible braiding phase is simply $\left\{\Theta_{1}^{\mathrm{H}}\right\}_{\mathbb{Z}_{N_{1}}}$. The corresponding TQFT action and gauge transformations are listed in Table I.

When $G=\prod_{i=1}^{2} \mathbb{Z}_{N_{i}}$, besides particle-loop braidings, three-loop braidings are realizable. In this case, all braiding processes are compatible with each other. The set of compatible braiding phase is the collection of all possible braiding

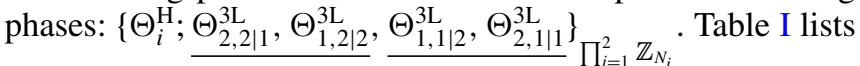
the TQFT action corresponding to compatible braiding phases along with its gauge transformations.

As for $G=\prod_{i=1}^{3} \mathbb{Z}_{N_{i}}$, the possible braiding processes are particle-loop braidings, three-loop braidings, and BR braidings. We find that particle-loop braidings are compatible with three-loop braidings or BR braidings, respectively. However, incompatibility occurs between three-loop braidings and BR braidings, even between different BR braidings. In general notations, a BR braiding phase $\Theta_{i, j \mid k}^{\mathrm{BR}}$ is incompatible with three-loop braiding phases $\Theta_{n, o \mid m}^{3 \mathrm{~L}}$ in which one of the indices $\{m, n, o\}$ is equal to $k$. Moreover, in the case of $G=\prod_{i=1}^{3} \mathbb{Z}_{N_{i}}$, $\Theta_{i, j \mid k}^{\mathrm{BR}}$ is incompatible with $\Theta_{r, s \mid t}^{\mathrm{BR}}(k \neq t)$ if $k=r$ or $k=s$. From the viewpoint of TQFT, such incompatibility can be explained by that there are no proper gauge transformations for the TQFT action, which consists of topological terms corresponding to incompatible braiding processes. Compatible braiding phases and corresponding TQFT actions of $G=$ $\prod_{i=1}^{3} \mathbb{Z}_{N_{i}}$ are summarized in Table II.

For the case of $G=\prod_{i=1}^{4} \mathbb{Z}_{N_{i}}$, besides the braidings mentioned above, four-loop braiding is realizable, classified as one of multiloop braidings. Similar to the case of $G=\prod_{i=1}^{3} \mathbb{Z}_{N_{i}}$, when $G=\prod_{i=1}^{4} \mathbb{Z}_{N_{i}}$, particle-loop braidings are compatible with multiloop braidings or BR braidings, respectively. However, the incompatibility between BR braidings and multiloop braidings still exists. $\Theta_{i, j \mid k}^{\mathrm{BR}}$ is only compatible with those $\Theta_{s, t \mid r}^{3 \mathrm{~L}}$ in which none of the indices $\{s, t, r\}$ is equal to $k$. No $\Theta_{i, j \mid k}^{\mathrm{BR}}$ is compatible with $\Theta_{1,2,3,4}^{4 \mathrm{~L}}$. Different from the case of
$G=\prod_{i=1}^{3} \mathbb{Z}_{N_{i}}$, when $G=\prod_{i=1}^{4} \mathbb{Z}_{N_{i}}$, different BR braidings may be compatible: For example, $\left\{\Theta_{1,2 \mid 4}^{\mathrm{BR}}, \Theta_{1,3 \mid 4}^{\mathrm{BR}}, \Theta_{2,3 \mid 4}^{\mathrm{BR}}\right\}$ and $\left\{\Theta_{1,2 \mid 3}^{\mathrm{BR}}, \Theta_{1,2 \mid 4}^{\mathrm{BR}}\right\}$ is, respectively, a subset of the sets of compatible braiding phases. Tables III and IV list all possible sets of compatible braiding phases, legitimate TQFT actions, and corresponding gauge transformations in the case of $G=$ $\prod_{i=1}^{4} \mathbb{Z}_{N_{i}}$.

Motivated by the compatibility of braiding processes in $(3+1) \mathrm{D}$ spacetime, we initiate an attempt to the physics of braiding processes in $(4+1) \mathrm{D}$ spacetime and their compatibility. Several results are given in Sec. V. A comprehensive understanding of compatible braiding processes and TQFTs in $(4+1) \mathrm{D}$ topological orders will be discussed in Ref. [61].

There could be several interesting future investigations based on the compatibility analysis of braiding phases. For example, it would be interesting to study the connection between incompatibility and quantum anomaly [32]. Meanwhile, we have not considered the $B B$ term [47,50,62-65], which can switch self-statistics (either bosonic or fermionic) of particles. In this sense, adding a $B B$ term may be useful when considering compatible braiding phases of either fermionic systems or bosonic systems with fermionic particle excitations that carry nontrivial gauge charges. Our work focuses only on topological orders where all particles are bosonic, so it is unnecessary to consider $B B$. In addition, $B B$ can drastically change the gauge group. In other words, the gauge group $G$ is simultaneously determined by the coefficients of the $B F$ term and $B B$ term. Nevertheless, this complexity leads to a superfluous difficulty in determining gauge group and does not provide new braidings among topological excitations. In addition, from experiences in two-dimensional topological orders, exhausting all non-Abelian braidings with non-Abelian gauge groups is very challenging if still applying the present field theory method. Algebraic tools from mathematics may be a much better way. It is definitely interesting to explore how to exhaust all legitimate topological orders with a non-Abelian gauge group.

\section{ACKNOWLEDGMENTS}

The authors would like to thank AtMa P.O. Chan, Shinsei Ryu, Zheng-Cheng Gu, Juven Wang, Meng Cheng, Eduardo Fradkin, Huan He, Apoorv Tiwari, and Xiao-Gang Wen for earlier discussions and/or collaborations on topological phases of matter in higher dimensions. This work was supported in part by the Sun Yat-sen University startup grant, Guangdong Basic and Applied Basic Research Foundation under Grant No. 2020B1515120100, National Natural Science Foundation of China (NSFC) Grants (No. 11847608 and No. 12074438).

\section{APPENDIX A: MICROSCOPIC DERIVATION OF THE TQFT ACTION $S \sim \int \sum_{i=1}^{3} B^{i} d A^{i}+A^{1} A^{2} B^{3}$}

We can derive the $\int B d A+A A B$ action from a threelayer $3 \mathrm{D}$ superfluid where layera 1 and 2 are in charge- $N_{1}$ and charge- $N_{2}$ particle condensations while layer 3 is in charge- $N_{3}$ vortexline condensation. To illustrate the vortexline 
condensation picture, we start with a normal superfluid state:

$$
\mathcal{L}_{3}=\frac{\rho_{3}}{2}\left(\partial_{\mu} \theta^{3}\right)^{2} \text {. }
$$

Separating the phase angle into a smooth part and singular part $\theta^{3}=\theta_{s}^{3}+\theta_{v}^{3}$ and introducing a Hubbard-Stratonovich auxiliary vector field $J_{\mu}^{3}$, the above $\mathcal{L}_{3}$ can be expressed as

$$
\mathcal{L}_{3}=\frac{1}{2 \rho_{3}}\left(J_{\mu}^{3}\right)^{2}+i J_{\mu}^{3}\left(\partial_{\mu} \theta_{s}^{3}+\partial_{\mu} \theta_{v}^{3}\right) .
$$

Integrating out the smooth part $\theta_{s}^{3}$ leads to a constraint $\partial_{\mu} J_{\mu}^{3}=$ 0 , which can be solved by introducing a two-form noncompact $\mathbb{U}(1)$ gauge field $B_{\mu \nu}^{3}$ :

$$
J_{\mu}^{3}=\frac{1}{4 \pi} \varepsilon^{\mu \nu \lambda \rho} \partial_{\nu} B_{\mu \nu}^{3} .
$$

Thus, $\mathcal{L}_{3}$ is dualed to the following gauge theory:

$$
\begin{aligned}
\mathcal{L}_{3} & =\frac{1}{2 \rho_{3}}\left(\frac{1}{4 \pi} \varepsilon^{\mu \nu \lambda \rho} \partial_{\nu} B_{\mu \nu}^{3}\right)^{2}+i \frac{1}{4 \pi} \varepsilon^{\mu \nu \lambda \rho} \partial_{\nu} B_{\mu \nu}^{3} \partial_{\mu} \theta_{v}^{3} \\
& =\frac{1}{2 \rho_{3}}\left(\frac{1}{4 \pi} \varepsilon^{\mu \nu \lambda \rho} \partial_{\nu} B_{\mu \nu}^{3}\right)^{2}+\frac{i}{2} B_{\mu \nu}^{3} \cdot \frac{1}{2 \pi} \varepsilon^{\mu \nu \lambda \rho} \partial_{\lambda} \partial_{\rho} \theta_{v}^{3} .
\end{aligned}
$$

The string current operator is defined by

$$
\Sigma_{\mu \nu}^{3}=\frac{1}{2 \pi} \varepsilon^{\mu \nu \lambda \rho} \partial_{\lambda} \partial_{\rho} \theta_{v}^{3} .
$$

Now consider a three-layer 3D superfluid where layers 1 and 2 are in particle condensation while layer 3 is in string condensation. The Lagrangian is

$$
\begin{aligned}
\mathcal{L}= & \frac{\rho_{3}}{2}\left(\partial_{[\mu} \Theta_{\nu]}-N_{3} B_{\mu \nu}^{3}\right)^{2}+\frac{\rho_{1}}{2}\left(\partial_{\mu} \theta^{1}-N_{1} A_{\mu}^{1}\right)^{2}+\frac{\rho_{2}}{2}\left(\partial_{\mu} \theta^{2}-N_{2} A_{\mu}^{2}\right)^{2} \\
& +i \Lambda \varepsilon^{\mu \nu \lambda \rho}\left(\partial_{[\mu} \Theta_{\nu]}-N_{3} B_{\mu \nu}^{3}\right)\left(\partial_{\lambda} \theta^{1}-N_{1} A_{\lambda}^{1}\right)\left(\partial_{\rho} \theta^{2}-N_{2} A_{\rho}^{2}\right)+\mathcal{L}_{\text {Maxwell }} \\
= & \frac{\rho_{3}}{2}\left(\partial_{[\mu} \Theta_{\nu]}-N_{3} B_{\mu \nu}^{3}\right)^{2}+\frac{\rho_{1}}{2}\left(\partial_{\mu} \theta^{1}-N_{1} A_{\mu}^{1}\right)^{2}+\frac{\rho_{2}}{2}\left(\partial_{\mu} \theta^{2}-N_{2} A_{\mu}^{2}\right)^{2}+\mathcal{L}_{\text {Maxwell }} \\
& +i \Lambda \varepsilon^{\mu \nu \lambda \rho} \partial_{[\mu} \Theta_{\nu]} \partial_{\lambda} \theta^{1} \partial_{\rho} \theta^{2}-i \Lambda \varepsilon^{\mu \nu \lambda \rho} \partial_{[\mu} \Theta_{\nu]} \partial_{\lambda} \theta^{1} N_{2} A_{\rho}^{2}-i \Lambda \varepsilon^{\mu \nu \lambda \rho} \partial_{[\mu} \Theta_{\nu]} \partial_{\rho} \theta^{2} N_{1} A_{\lambda}^{1}+i \Lambda \varepsilon^{\mu \nu \lambda \rho} \partial_{[\mu} \Theta_{\nu]} N_{1} A_{\lambda}^{1} N_{2} A_{\rho}^{2} \\
& -i \Lambda \varepsilon^{\mu \nu \lambda \rho} N_{3} B_{\mu \nu}^{3} \partial_{\lambda} \theta^{1} \partial_{\rho} \theta^{2}+i \Lambda \varepsilon^{\mu \nu \lambda \rho} N_{3} B_{\mu \nu}^{3} \partial_{\lambda} \theta^{1} N_{2} A_{\rho}^{2}+i \Lambda \varepsilon^{\mu \nu \lambda \rho} N_{3} B_{\mu \nu}^{3} \partial_{\rho} \theta^{2} N_{1} A_{\lambda}^{1}-i \Lambda \varepsilon^{\mu \nu \lambda \rho} N_{1} A_{\lambda}^{1} N_{2} A_{\rho}^{2} N_{3} B_{\mu \nu}^{3} \\
= & \frac{\rho_{3}}{2}\left(\partial_{[\mu} \Theta_{\nu]}-N_{3} B_{\mu \nu}^{3}\right)^{2}+\frac{\rho_{1}}{2}\left(\partial_{\mu} \theta^{1}-N_{1} A_{\mu}^{1}\right)^{2}+\frac{\rho_{2}}{2}\left(\partial_{\mu} \theta^{2}-N_{2} A_{\mu}^{2}\right)^{2}+\mathcal{L}_{\text {Maxwell }}+\text { boundary terms } \\
& +i \Lambda \varepsilon^{\mu \nu \lambda \rho}\left[2 \Theta_{\mu} \partial_{\nu}\left(\partial_{\lambda} \theta^{1} N_{2} A_{\rho}^{2}\right)\right]+i \Lambda \varepsilon^{\mu \nu \lambda \rho}\left[2 \Theta_{\mu} \partial_{\nu}\left(\partial_{\rho} \theta^{2} N_{1} A_{\lambda}^{1}\right)\right]+i \Lambda \varepsilon^{\mu \nu \lambda \rho} \frac{1}{2}\left[\theta^{1} \partial_{\lambda}\left(\partial_{\rho} \theta^{2} N_{3} B_{\mu \nu}^{3}\right)+\theta^{2} \partial_{\rho}\left(\partial_{\lambda} \theta^{1} N_{3} B_{\mu \nu}^{3}\right)\right] \\
& -i \Lambda \varepsilon^{\mu \nu \lambda \rho}\left[2 \Theta_{\mu} \partial_{\nu}\left(N_{1} N_{2} A_{\lambda}^{1} A_{\rho}^{2}\right)\right]+i \Lambda \varepsilon^{\mu \nu \lambda \rho}\left[-\theta^{1} \partial_{\lambda}\left(N_{2} A_{\rho}^{2} N_{3} B_{\mu \nu}^{3}\right)\right]+i \Lambda \varepsilon^{\mu \nu \lambda \rho}\left[-\theta^{2} \partial_{\rho}\left(N_{3} B_{\mu \nu}^{3} N_{1} A_{\lambda}^{1}\right)\right] \\
& -i \Lambda \varepsilon^{\mu \nu \lambda \rho} A_{\lambda}^{1} A_{\rho}^{2} N_{3} B_{\mu \nu}^{3},
\end{aligned}
$$

where $\partial_{[\mu} \Theta_{\nu]}=\partial_{\mu} \Theta_{\nu}-\partial_{\nu} \Theta_{\mu}$. Introducing Hubbard-Stratonovich fields $\Sigma_{\mu \nu}^{3}, j^{1}$ and $j^{2}$ :

$$
\begin{aligned}
\mathcal{L}= & \frac{1}{8 \rho_{3}}\left(\Sigma_{\mu \nu}^{3}\right)^{2}+i \frac{1}{2} \Sigma_{\mu \nu}^{3}\left(\partial_{[\mu} \Theta_{\nu]}-N_{3} B_{\mu \nu}^{3}\right)+\frac{1}{2 \rho_{1}}\left(j^{1}\right)^{2}+i j_{\lambda}^{1}\left(\partial_{\lambda} \theta^{1}-N_{1} A_{\lambda}^{1}\right)+\frac{1}{2 \rho_{2}}\left(j^{2}\right)^{2}+i j_{\rho}^{2}\left(\partial_{\rho} \theta^{2}-N_{2} A_{\rho}^{2}\right) \\
& +i \Lambda \varepsilon^{\mu \nu \lambda \rho}\left[2 \Theta_{\mu} \partial_{\nu}\left(\partial_{\lambda} \theta^{1} N_{2} A_{\rho}^{2}\right)\right]+i \Lambda \varepsilon^{\mu \nu \lambda \rho}\left[2 \Theta_{\mu} \partial_{\nu}\left(\partial_{\rho} \theta^{2} N_{1} A_{\lambda}^{1}\right)\right]+i \Lambda \varepsilon^{\mu \nu \lambda \rho} \frac{1}{2}\left[\theta^{1} \partial_{\lambda}\left(\partial_{\rho} \theta^{2} N_{3} B_{\mu \nu}^{3}\right)+\theta^{2} \partial_{\rho}\left(\partial_{\lambda} \theta^{1} N_{3} B_{\mu \nu}^{3}\right)\right] \\
& -i \Lambda \varepsilon^{\mu \nu \lambda \rho}\left[2 \Theta_{\mu} \partial_{\nu}\left(N_{1} N_{2} A_{\lambda}^{1} A_{\rho}^{2}\right)\right]+i \Lambda \varepsilon^{\mu \nu \lambda \rho}\left[-\theta^{1} \partial_{\lambda}\left(N_{2} A_{\rho}^{2} N_{3} B_{\mu \nu}^{3}\right)\right]+i \Lambda \varepsilon^{\mu \nu \lambda \rho}\left[-\theta^{2} \partial_{\rho}\left(N_{3} B_{\mu \nu}^{3} N_{1} A_{\lambda}^{1}\right)\right] \\
& -i N_{1} N_{2} N_{3} \Lambda \varepsilon^{\mu \nu \lambda \rho} A_{\lambda}^{1} A_{\rho}^{2} B_{\mu \nu}^{3}+\mathcal{L}_{\text {Maxwell }}+\text { boundary terms. }
\end{aligned}
$$

Further introduce Lagrange multiplier fields $\xi^{I}$ and $\eta^{I}$ to decouple terms like $i \Lambda \varepsilon^{\mu \nu \lambda \rho}\left[-\theta^{1} \partial_{\lambda}\left(\partial_{\rho} \theta^{2} N_{3} B_{\mu \nu}^{3}\right)\right]$ :

$$
\begin{aligned}
\mathcal{L}= & \frac{1}{8 \rho_{3}}\left(\Sigma_{\mu \nu}^{3}\right)^{2}-i \Theta_{\mu} \partial_{\nu} \Sigma_{\mu \nu}^{3}-i \frac{1}{2} N_{3} B_{\mu \nu}^{3} \Sigma_{\mu \nu}^{3}+\frac{1}{2 \rho_{1}}\left(j^{1}\right)^{2}-i \theta^{1} \partial_{\lambda} j_{\lambda}^{1}-i N_{1} A_{\lambda}^{1} j_{\lambda}^{1}+\frac{1}{2 \rho_{2}}\left(j^{2}\right)^{2}-i \theta^{2} \partial_{\rho} j_{\rho}^{2}-i N_{2} A_{\rho}^{2} j_{\rho}^{2} \\
& +i \Lambda \varepsilon^{\mu \nu \lambda \rho}\left[2 \Theta_{\mu} \partial_{\nu}\left(N_{1} N_{2} A_{\lambda}^{1} A_{\rho}^{2}\right)+\theta^{1} \partial_{\lambda}\left(N_{2} A_{\rho}^{2} N_{3} B_{\mu \nu}^{3}\right)+\theta^{2} \partial_{\rho}\left(N_{3} B_{\mu \nu}^{3} N_{1} A_{\lambda}^{1}\right)\right]-i N_{1} N_{2} N_{3} \Lambda \varepsilon^{\mu \nu \lambda \rho} A_{\lambda}^{1} A_{\rho}^{2} B_{\mu \nu}^{3} \\
& +i \eta_{\lambda}^{1}\left[\xi_{\lambda}^{1}-\Lambda \varepsilon^{\mu \nu \lambda \rho} \cdot \frac{1}{2} \partial_{\rho} \theta^{2} N_{3} B_{\mu \nu}^{3}\right]+i \theta^{1} \partial_{\lambda} \xi_{\lambda}^{1}+i \eta_{\rho}^{2}\left[\xi_{\rho}^{2}-\Lambda \varepsilon^{\mu \nu \lambda \rho} \cdot \frac{1}{2} \partial_{\lambda} \theta^{1} N_{3} B_{\mu \nu}^{3}\right]+i \theta^{2} \partial_{\rho} \xi_{\rho}^{2} \\
& +i \eta_{\mu \nu}^{3}\left[\xi_{\mu \nu}^{3}-\Lambda \varepsilon^{\mu \nu \lambda \rho}\left(2 \partial_{\lambda} \theta^{1} N_{2} A_{\rho}^{2}+2 \partial_{\rho} \theta^{2} N_{1} A_{\lambda}^{1}\right)\right]+i \Theta_{\mu} \partial_{\nu} \xi_{\mu \nu}^{3}+\mathcal{L}_{\text {Maxwell }}+\text { boundary terms } \\
= & \frac{1}{8 \rho_{3}}\left(\Sigma_{\mu \nu}^{3}\right)^{2}+\frac{1}{2 \rho_{1}}\left(j^{1}\right)^{2}+\frac{1}{2 \rho_{2}}\left(j^{2}\right)^{2}-i \frac{1}{2} N_{3} B_{\mu \nu}^{3} \Sigma_{\mu \nu}^{3}-i N_{1} A_{\lambda}^{1} j_{\lambda}^{1}-i N_{2} A_{\rho}^{2} j_{\rho}^{2} \\
& -i \Theta_{\mu} \partial_{\nu}\left[\Sigma_{\mu \nu}^{3}-\xi_{\mu \nu}^{3}+\Lambda \varepsilon^{\mu \nu \lambda \rho} \cdot 2 N_{1} N_{2} A_{\lambda}^{1} A_{\rho}^{2}\right]+i \eta_{\mu \nu}^{3} \xi_{\mu \nu}^{3}
\end{aligned}
$$




$$
\begin{aligned}
& -i \theta^{1} \partial_{\lambda}\left[j_{\lambda}^{1}-\xi_{\lambda}^{1}+\Lambda \varepsilon^{\mu \nu \lambda \rho}\left(N_{2} A_{\rho}^{2} N_{3} B_{\mu \nu}^{3}-\frac{1}{2} \eta_{\rho}^{2} N_{3} B_{\mu \nu}^{3}-2 \eta_{\mu \nu}^{3} N_{2} A_{\rho}^{2}\right)\right]+i \eta_{\lambda}^{1} \xi_{\lambda}^{1} \\
& -i \theta^{2} \partial_{\rho}\left[j_{\rho}^{2}-\xi_{\rho}^{2}+\Lambda \varepsilon^{\mu \nu \lambda \rho}\left(N_{3} B_{\mu \nu}^{3} N_{1} A_{\lambda}^{1}-\frac{1}{2} \eta_{\lambda}^{1} N_{3} B_{\mu \nu}^{3}-2 \eta_{\mu \nu}^{3} N_{1} A_{\lambda}^{1}\right)\right]+i \eta_{\rho}^{1} \xi_{\rho}^{1} \\
& -i N_{1} N_{2} N_{3} \Lambda \varepsilon^{\mu \nu \lambda \rho} A_{\lambda}^{1} A_{\rho}^{2} B_{\mu \nu}^{3}+\mathcal{L}_{\text {Maxwell }}+\text { boundary terms. }
\end{aligned}
$$

Integrating out $\Theta_{\mu}, \theta^{1}$, and $\theta^{2}$ yields constraints

$$
\begin{gathered}
\partial_{\nu}\left[\Sigma_{\mu \nu}^{3}-\xi_{\mu \nu}^{3}+\Lambda \varepsilon^{\mu \nu \lambda \rho} \cdot 2 N_{1} N_{2} A_{\lambda}^{1} A_{\rho}^{2}\right]=0, \\
\partial_{\lambda}\left[j_{\lambda}^{1}-\xi_{\lambda}^{1}+\Lambda \varepsilon^{\mu \nu \lambda \rho}\left(N_{2} A_{\rho}^{2} N_{3} B_{\mu \nu}^{3}-\frac{1}{2} \eta_{\rho}^{2} N_{3} B_{\mu \nu}^{3}-2 \eta_{\mu \nu}^{3} N_{2} A_{\rho}^{2}\right)\right]=0,
\end{gathered}
$$

and

$$
\partial_{\rho}\left[j_{\rho}^{2}-\xi_{\rho}^{2}+\Lambda \varepsilon^{\mu \nu \lambda \rho}\left(N_{3} B_{\mu \nu}^{3} N_{1} A_{\lambda}^{1}-\frac{1}{2} \eta_{\lambda}^{1} N_{3} B_{\mu \nu}^{3}-2 \eta_{\mu \nu}^{3} N_{1} A_{\lambda}^{1}\right)\right]=0 .
$$

These constraints can be solved by

$$
\begin{gathered}
\Sigma_{\mu \nu}^{3}=\frac{1}{2 \pi} \varepsilon^{\mu \nu \lambda \rho} \partial_{\lambda} A_{\rho}^{3}+\xi_{\mu \nu}^{3}-\Lambda \varepsilon^{\mu \nu \lambda \rho} \cdot 2 N_{1} N_{2} A_{\lambda}^{1} A_{\rho}^{2}, \\
j_{\lambda}^{1}=\frac{1}{4 \pi} \varepsilon^{\lambda \rho \mu \nu} \partial_{\rho} B_{\mu \nu}^{1}+\xi_{\lambda}^{1}-\Lambda \varepsilon^{\mu \nu \lambda \rho}\left(N_{2} A_{\rho}^{2} N_{3} B_{\mu \nu}^{3}-\frac{1}{2} \eta_{\rho}^{2} N_{3} B_{\mu \nu}^{3}-2 \eta_{\mu \nu}^{3} N_{2} A_{\rho}^{2}\right), \\
j_{\rho}^{2}=\frac{1}{4 \pi} \varepsilon^{\rho \lambda \mu \nu} \partial_{\lambda} B_{\mu \nu}^{2}+\xi_{\rho}^{2}-\Lambda \varepsilon^{\mu \nu \lambda \rho}\left(N_{3} B_{\mu \nu}^{3} N_{1} A_{\lambda}^{1}-\frac{1}{2} \eta_{\lambda}^{1} N_{3} B_{\mu \nu}^{3}-2 \eta_{\mu \nu}^{3} N_{1} A_{\lambda}^{1}\right) .
\end{gathered}
$$

Then we obtain

$$
\begin{aligned}
\mathcal{L}= & -i \frac{1}{2} N_{3} B_{\mu \nu}^{3}\left[\frac{1}{2 \pi} \varepsilon^{\mu \nu \lambda \rho} \partial_{\lambda} A_{\rho}^{3}+\xi_{\mu \nu}^{3}-\Lambda \varepsilon^{\mu \nu \lambda \rho} \cdot 2 N_{1} N_{2} A_{\lambda}^{1} A_{\rho}^{2}\right] \\
& -i N_{1} A_{\lambda}^{1}\left[\frac{1}{4 \pi} \varepsilon^{\lambda \rho \mu \nu} \partial_{\rho} B_{\mu \nu}^{1}+\xi_{\lambda}^{1}-\Lambda \varepsilon^{\mu \nu \lambda \rho}\left(N_{2} A_{\rho}^{2} N_{3} B_{\mu \nu}^{3}-\frac{1}{2} \eta_{\rho}^{2} N_{3} B_{\mu \nu}^{3}-2 \eta_{\mu \nu}^{3} N_{2} A_{\rho}^{2}\right)\right] \\
& -i N_{2} A_{\rho}^{2}\left[\frac{1}{4 \pi} \varepsilon^{\rho \lambda \mu \nu} \partial_{\lambda} B_{\mu \nu}^{2}+\xi_{\rho}^{2}-\Lambda \varepsilon^{\mu \nu \lambda \rho}\left(N_{3} B_{\mu \nu}^{3} N_{1} A_{\lambda}^{1}-\frac{1}{2} \eta_{\lambda}^{1} N_{3} B_{\mu \nu}^{3}-2 \eta_{\mu \nu}^{3} N_{1} A_{\lambda}^{1}\right)\right] \\
& +\frac{1}{8 \rho_{3}}\left[\frac{1}{2 \pi} \varepsilon^{\mu \nu \lambda \rho} \partial_{\lambda} A_{\rho}^{3}+\xi_{\mu \nu}^{3}-\Lambda \varepsilon^{\mu \nu \lambda \rho} \cdot 2 N_{1} N_{2} A_{\lambda}^{1} A_{\rho}^{2}\right]^{2} \\
& +\frac{1}{2 \rho_{1}}\left[\frac{1}{4 \pi} \varepsilon^{\lambda \rho \mu \nu} \partial_{\rho} B_{\mu \nu}^{1}+\xi_{\lambda}^{1}-\Lambda \varepsilon^{\mu \nu \lambda \rho}\left(N_{2} A_{\rho}^{2} N_{3} B_{\mu \nu}^{3}-\frac{1}{2} \eta_{\rho}^{2} N_{3} B_{\mu \nu}^{3}-2 \eta_{\mu \nu}^{3} N_{2} A_{\rho}^{2}\right)\right]^{2} \\
& +\frac{1}{2 \rho_{2}}\left[\frac{1}{4 \pi} \varepsilon^{\rho \lambda \mu \nu} \partial_{\lambda} B_{\mu \nu}^{2}+\xi_{\rho}^{2}-\Lambda \varepsilon^{\mu \nu \lambda \rho}\left(N_{3} B_{\mu \nu}^{3} N_{1} A_{\lambda}^{1}-\frac{1}{2} \eta_{\lambda}^{1} N_{3} B_{\mu \nu}^{3}-2 \eta_{\mu \nu}^{3} N_{1} A_{\lambda}^{1}\right)\right]^{2} \\
& +i \eta_{\lambda}^{1} \xi_{\lambda}^{1}+i \eta_{\rho}^{2} \xi_{\rho}^{2}+i \eta_{\mu}^{3} \xi_{\mu \nu}^{3}-i N_{1} N_{2} N_{3} \Lambda \varepsilon^{\mu \nu \lambda \rho} A_{\lambda}^{1} A_{\rho}^{2} B_{\mu \nu}^{3}+\mathcal{L}_{\text {Maxwell }}+\text { boundary terms. }
\end{aligned}
$$

Let us write $\mathcal{L}=\mathcal{L}_{A^{1}}+\mathcal{L}_{A^{2}}+\mathcal{L}_{B^{3}}-i N_{1} N_{2} N_{3} \Lambda \varepsilon^{\mu \nu \lambda \rho} A_{\lambda}^{1} A_{\rho}^{2} B_{\mu \nu}^{3}+\mathcal{L}_{\text {Maxwell }}$, where

$$
\begin{aligned}
\mathcal{L}_{B^{3}}= & -\frac{i N_{3}}{4 \pi} \varepsilon^{\mu \nu \lambda \rho} B_{\mu \nu}^{3} \partial_{\lambda} A_{\rho}^{3}+i \frac{1}{2} \Lambda \varepsilon^{\mu \nu \lambda \rho} N_{3} B_{\mu \nu}^{3} \cdot 2 N_{1} N_{2} A_{\lambda}^{1} A_{\rho}^{2}+\frac{1}{8 \rho_{3}}\left[\frac{1}{2 \pi} \varepsilon^{\mu \nu \lambda \rho} \partial_{\lambda} A_{\rho}^{3}-\Lambda \varepsilon^{\mu \nu \lambda \rho} \cdot 2 N_{1} N_{2} A_{\lambda}^{1} A_{\rho}^{2}\right]^{2}+\frac{1}{8 \rho_{3}}\left(\xi_{\mu \nu}^{3}\right)^{2} \\
& +\frac{1}{4 \rho_{3}} \xi_{\mu \nu}^{3}\left[i 2 \rho_{3}\left(2 \eta_{\mu \nu}^{3}-N_{3} B_{\mu \nu}^{3}\right)+\frac{1}{2 \pi} \varepsilon^{\mu \nu \lambda \rho} \partial_{\lambda} A_{\rho}^{3}-\Lambda \varepsilon^{\mu \nu \lambda \rho} \cdot 2 N_{1} N_{2} A_{\lambda}^{1} A_{\rho}^{2}\right] \\
\mathcal{L}_{A^{1}}= & -\frac{i N_{1}}{4 \pi} \varepsilon^{\lambda \rho \mu \nu} A_{\lambda}^{1} \partial_{\rho} B_{\mu \nu}^{1}+i \Lambda \varepsilon^{\mu \nu \lambda \rho} N_{1} A_{\lambda}^{1}\left(N_{2} A_{\rho}^{2} N_{3} B_{\mu \nu}^{3}-\frac{1}{2} \eta_{\rho}^{2} N_{3} B_{\mu \nu}^{3}-2 \eta_{\mu \nu}^{3} N_{2} A_{\rho}^{2}\right) \\
& +\frac{1}{2 \rho_{1}}\left[\frac{1}{4 \pi} \varepsilon^{\lambda \rho \mu \nu} \partial_{\rho} B_{\mu \nu}^{1}-\Lambda \varepsilon^{\mu \nu \lambda \rho}\left(N_{2} A_{\rho}^{2} N_{3} B_{\mu \nu}^{3}-\frac{1}{2} \eta_{\rho}^{2} N_{3} B_{\mu \nu}^{3}-2 \eta_{\mu \nu}^{3} N_{2} A_{\rho}^{2}\right)\right]^{2}+\frac{1}{2 \rho_{1}}\left(\xi_{\lambda}^{1}\right)^{2} \\
& +\frac{1}{\rho_{1}} \xi_{\lambda}^{1}\left[i \rho_{1}\left(\eta_{\lambda}^{1}-N_{1} A_{\lambda}^{1}\right)+\frac{1}{4 \pi} \varepsilon^{\lambda \rho \mu \nu} \partial_{\rho} B_{\mu \nu}^{1}-\Lambda \varepsilon^{\mu \nu \lambda \rho}\left(N_{2} A_{\rho}^{2} N_{3} B_{\mu \nu}^{3}-\frac{1}{2} \eta_{\rho}^{2} N_{3} B_{\mu \nu}^{3}-2 \eta_{\mu \nu}^{3} N_{2} A_{\rho}^{2}\right)\right]
\end{aligned}
$$




$$
\begin{aligned}
\mathcal{L}_{A^{2}}= & -\frac{i N_{2}}{4 \pi} \varepsilon^{\mu \nu \lambda \rho} A_{\rho}^{2} \partial_{\lambda} B_{\mu \nu}^{2}+i \Lambda \varepsilon^{\mu \nu \lambda \rho} N_{2} A_{\rho}^{2}\left(N_{3} B_{\mu \nu}^{3} N_{1} A_{\lambda}^{1}-\frac{1}{2} \eta_{\lambda}^{1} N_{3} B_{\mu \nu}^{3}-2 \eta_{\mu \nu}^{3} N_{1} A_{\lambda}^{1}\right) \\
& +\frac{1}{2 \rho_{2}}\left[\frac{1}{4 \pi} \varepsilon^{\rho \lambda \mu \nu} \partial_{\lambda} B_{\mu \nu}^{2}-\Lambda \varepsilon^{\mu \nu \lambda \rho}\left(N_{3} B_{\mu \nu}^{3} N_{1} A_{\lambda}^{1}-\frac{1}{2} \eta_{\lambda}^{1} N_{3} B_{\mu \nu}^{3}-2 \eta_{\mu \nu}^{3} N_{1} A_{\lambda}^{1}\right)\right]+\frac{1}{2 \rho_{2}}\left(\xi_{\rho}^{2}\right)^{2} \\
& +\frac{1}{\rho_{2}} \xi_{\rho}^{2}\left[i \rho_{2}\left(\eta_{\rho}^{2}-N_{2} A_{\rho}^{2}\right)+\frac{1}{4 \pi} \varepsilon^{\rho \lambda \mu \nu} \partial_{\lambda} B_{\mu \nu}^{2}-\Lambda \varepsilon^{\mu \nu \lambda \rho}\left(N_{3} B_{\mu \nu}^{3} N_{1} A_{\lambda}^{1}-\frac{1}{2} \eta_{\lambda}^{1} N_{3} B_{\mu \nu}^{3}-2 \eta_{\mu \nu}^{3} N_{1} A_{\lambda}^{1}\right)\right] .
\end{aligned}
$$

Integrate out $\xi_{\mu \nu}^{3}$ :

$$
\begin{aligned}
\mathcal{L}_{B_{3}}= & -\frac{i N_{3}}{4 \pi} \varepsilon^{\mu \nu \lambda \rho} B_{\mu \nu}^{3} \partial_{\lambda} A_{\rho}^{3}+i \frac{1}{2} \Lambda \varepsilon^{\mu \nu \lambda \rho} N_{3} B_{\mu \nu}^{3} \cdot 2 N_{1} N_{2} A_{\lambda}^{1} A_{\rho}^{2}+\frac{1}{8 \rho_{3}}\left[\frac{1}{2 \pi} \varepsilon^{\mu \nu \lambda \rho} \partial_{\lambda} A_{\rho}^{3}-\Lambda \varepsilon^{\mu \nu \lambda \rho} \cdot 2 N_{1} N_{2} A_{\lambda}^{1} A_{\rho}^{2}\right]^{2} \\
& -\frac{1}{8 \rho_{3}}\left[i 2 \rho_{3}\left(2 \eta_{\mu \nu}^{3}-N_{3} B_{\mu \nu}^{3}\right)+\frac{1}{2 \pi} \varepsilon^{\mu \nu \lambda \rho} \partial_{\lambda} N_{3} A_{\rho}^{3}-\Lambda \varepsilon^{\mu \nu \lambda \rho} \cdot 2 N_{1} N_{2} A_{\lambda}^{1} A_{\rho}^{2}\right]^{2} \\
= & -\frac{i N_{3}}{4 \pi} \varepsilon^{\mu \nu \lambda \rho} B_{\mu \nu}^{3} \partial_{\lambda} A_{\rho}^{3}+i N_{1} N_{2} N_{3} \Lambda \varepsilon^{\mu \nu \lambda \rho} B_{\mu \nu}^{3} A_{\lambda}^{1} A_{\rho}^{2} \\
& +\frac{\rho_{3}}{2}\left(2 \eta_{\mu \nu}^{3}-N_{3} B_{\mu \nu}^{3}\right)^{2}-i \frac{1}{2}\left(2 \eta_{\mu \mu}^{3}-N_{3} B_{\mu \nu}^{3}\right)\left(\frac{1}{2 \pi} \varepsilon^{\mu \nu \lambda \rho} \partial_{\lambda} A_{\rho}^{3}-\Lambda \varepsilon^{\mu \nu \lambda \rho} \cdot 2 N_{1} N_{2} A_{\lambda}^{1} A_{\rho}^{2}\right) \\
= & \frac{\rho_{3}}{2}\left(2 \eta_{\mu \nu}^{3}-N_{3} B_{\mu \nu}^{3}\right)^{2}-\frac{i}{2 \pi} \varepsilon^{\mu \nu \lambda \rho} \eta_{\mu \nu}^{3} \partial_{\lambda} A_{\rho}^{3}+i \Lambda \varepsilon^{\mu \nu \lambda \rho} \eta_{\mu \nu}^{3} \cdot 2 N_{1} N_{2} A_{\lambda}^{1} A_{\rho}^{2} .
\end{aligned}
$$

Integrate out $\xi_{\lambda}^{1}$ :

$$
\begin{aligned}
\mathcal{L}_{A^{1}}= & -\frac{i N_{1}}{4 \pi} \varepsilon^{\lambda \rho \mu \nu} A_{\lambda}^{1} \partial_{\rho} B_{\mu \nu}^{1}+i \Lambda \varepsilon^{\mu \nu \lambda \rho} N_{1} A_{\lambda}^{1}\left(N_{2} A_{\rho}^{2} N_{3} B_{\mu \nu}^{3}-\frac{1}{2} \eta_{\rho}^{2} N_{3} B_{\mu \nu}^{3}-2 \eta_{\mu \nu}^{3} N_{2} A_{\rho}^{2}\right) \\
& +\frac{1}{2 \rho_{1}}\left[\frac{1}{4 \pi} \varepsilon^{\lambda \rho \mu \nu} \partial_{\rho} B_{\mu \nu}^{1}-\Lambda \varepsilon^{\mu \nu \lambda \rho}\left(N_{2} A_{\rho}^{2} N_{3} B_{\mu \nu}^{3}-\frac{1}{2} \eta_{\rho}^{2} N_{3} B_{\mu \nu}^{3}-2 \eta_{\mu \nu}^{3} N_{2} A_{\rho}^{2}\right)\right]^{2} \\
& -\frac{1}{2 \rho_{1}}\left[i \rho\left(\eta_{\lambda}^{1}-N_{1} A_{\lambda}^{1}\right)+\frac{1}{4 \pi} \varepsilon^{\lambda \rho \mu \nu} \partial_{\rho} B_{\mu \nu}^{1}-\Lambda \varepsilon^{\mu \nu \lambda \rho}\left(N_{2} A_{\rho}^{2} N_{3} B_{\mu \nu}^{3}-\frac{1}{2} \eta_{\rho}^{2} N_{3} B_{\mu \nu}^{3}-2 \eta_{\mu \nu}^{3} N_{2} A_{\rho}^{2}\right)\right]^{2} \\
= & -\frac{i N_{1}}{4 \pi} \varepsilon^{\lambda \rho \mu \nu} A_{\lambda}^{1} \partial_{\rho} B_{\mu \nu}^{1}+i \Lambda \varepsilon^{\mu \nu \lambda \rho} N_{1} A_{\lambda}^{1}\left(N_{2} A_{\rho}^{2} N_{3} B_{\mu \nu}^{3}-\frac{1}{2} \eta_{\rho}^{2} N_{3} B_{\mu \nu}^{3}-2 \eta_{\mu \nu}^{3} N_{2} A_{\rho}^{2}\right) \\
& +\frac{\rho_{1}}{2}\left(\eta_{\lambda}^{1}-N_{1} A_{\lambda}^{1}\right)^{2}-i\left(\eta_{\lambda}^{1}-N_{1} A_{\lambda}^{1}\right)\left[\frac{1}{4 \pi} \varepsilon^{\lambda \rho \mu \nu} \partial_{\rho} B_{\mu \nu}^{1}-\Lambda \varepsilon^{\mu \nu \lambda \rho}\left(N_{2} A_{\rho}^{2} N_{3} B_{\mu \nu}^{3}-\frac{1}{2} \eta_{\rho}^{2} N_{3} B_{\mu \nu}^{3}-2 \eta_{\mu \nu}^{3} N_{2} A_{\rho}^{2}\right)\right] \\
= & \frac{\rho_{1}}{2}\left(\eta_{\lambda}^{1}-A_{\lambda}^{1}\right)^{2}-\frac{i}{4 \pi} \varepsilon^{\lambda \rho \mu \nu} \eta_{\lambda}^{1} \partial_{\rho} B_{\mu \nu}^{1}+i \Lambda \varepsilon^{\mu \nu \lambda \rho} \eta_{\lambda}^{1}\left(N_{2} A_{\rho}^{2} N_{3} B_{\mu \nu}^{3}-\frac{1}{2} \eta_{\rho}^{2} N_{3} B_{\mu \nu}^{3}-2 \eta_{\mu \nu}^{3} N_{2} A_{\rho}^{2}\right) .
\end{aligned}
$$

Integrate out $\xi_{\rho}^{2}$ :

$$
\begin{aligned}
\mathcal{L}_{A^{2}}= & -\frac{i N_{2}}{4 \pi} \varepsilon^{\mu \nu \lambda \rho} A_{\rho}^{2} \partial_{\lambda} B_{\mu \nu}^{2}+i \Lambda \varepsilon^{\mu \nu \lambda \rho} N_{2} A_{\rho}^{2}\left(N_{3} B_{\mu \nu}^{3} N_{1} A_{\lambda}^{1}-\frac{1}{2} \eta_{\lambda}^{1} N_{3} B_{\mu \nu}^{3}-2 \eta_{\mu \nu}^{3} N_{1} A_{\lambda}^{1}\right) \\
& +\frac{1}{2 \rho_{2}}\left[\frac{1}{4 \pi} \varepsilon^{\rho \lambda \mu \nu} \partial_{\lambda} B_{\mu \nu}^{2}-\Lambda \varepsilon^{\mu \nu \lambda \rho}\left(N_{3} B_{\mu \nu}^{3} N_{1} A_{\lambda}^{1}-\frac{1}{2} \eta_{\lambda}^{1} N_{3} B_{\mu \nu}^{3}-2 \eta_{\mu \nu}^{3} N_{1} A_{\lambda}^{1}\right)\right] \\
& -\frac{1}{2 \rho_{2}}\left[i \rho_{2}\left(\eta_{\rho}^{2}-N_{2} A_{\rho}^{2}\right)+\frac{1}{4 \pi} \varepsilon^{\rho \lambda \mu \nu} \partial_{\lambda} B_{\mu \nu}^{2}-\Lambda \varepsilon^{\mu \nu \lambda \rho}\left(N_{3} B_{\mu \nu}^{3} N_{1} A_{\lambda}^{1}-\frac{1}{2} \eta_{\lambda}^{1} N_{3} B_{\mu \nu}^{3}-2 \eta_{\mu \nu}^{3} N_{1} A_{\lambda}^{1}\right)\right]^{2} \\
= & -\frac{i N_{2}}{4 \pi} \varepsilon^{\mu \nu \lambda \rho} A_{\rho}^{2} \partial_{\lambda} B_{\mu \nu}^{2}+i \Lambda \varepsilon^{\mu \nu \lambda \rho} N_{2} A_{\rho}^{2}\left(N_{3} B_{\mu \nu}^{3} N_{1} A_{\lambda}^{1}-\frac{1}{2} \eta_{\lambda}^{1} N_{3} B_{\mu \nu}^{3}-2 \eta_{\mu \nu}^{3} N_{1} A_{\lambda}^{1}\right) \\
& +\frac{\rho_{2}}{2}\left(\eta_{\rho}^{2}-N_{2} A_{\rho}^{2}\right)^{2}-i\left(\eta_{\rho}^{2}-N_{2} A_{\rho}^{2}\right)\left[\frac{1}{4 \pi} \varepsilon^{\rho \lambda \mu \nu} \partial_{\lambda} B_{\mu \nu}^{2}-\Lambda \varepsilon^{\mu \nu \lambda \rho}\left(N_{3} B_{\mu \nu}^{3} N_{1} A_{\lambda}^{1}-\frac{1}{2} \eta_{\lambda}^{1} N_{3} B_{\mu \nu}^{3}-2 \eta_{\mu \nu}^{3} N_{1} A_{\lambda}^{1}\right)\right] \\
= & \frac{\rho_{2}}{2}\left(\eta_{\rho}^{2}-N_{2} A_{\rho}^{2}\right)^{2}-\frac{i}{4 \pi} \varepsilon^{\rho \lambda \mu \nu} \eta_{\rho}^{2} \partial_{\lambda} B_{\mu \nu}^{2}+i \Lambda \varepsilon^{\mu \nu \lambda \rho} \eta_{\rho}^{2}\left(N_{3} B_{\mu \nu}^{3} N_{1} A_{\lambda}^{1}-\frac{1}{2} \eta_{\lambda}^{1} N_{3} B_{\mu \nu}^{3}-2 \eta_{\mu \nu}^{3} N_{1} A_{\lambda}^{1}\right) .
\end{aligned}
$$


We end up with

$$
\begin{aligned}
\mathcal{L}= & \frac{\rho_{3}}{2}\left(2 \eta_{\mu \nu}^{3}-N_{3} B_{\mu \nu}^{3}\right)^{2}-\frac{i}{2 \pi} \varepsilon^{\mu \nu \lambda \rho} \eta_{\mu \nu}^{3} \partial_{\lambda} A_{\rho}^{3}+i \Lambda \varepsilon^{\mu \nu \lambda \rho} \eta_{\mu \nu}^{3} \cdot 2 N_{1} N_{2} A_{\lambda}^{1} A_{\rho}^{2}+\frac{\rho_{1}}{2}\left(\eta_{\lambda}^{1}-N_{1} A_{\lambda}^{1}\right)^{2}-\frac{i}{4 \pi} \varepsilon^{\lambda \rho \mu \nu} \eta_{\lambda}^{1} \partial_{\rho} B_{\mu \nu}^{1} \\
& +i \Lambda \varepsilon^{\mu \nu \lambda \rho} \eta_{\lambda}^{1}\left(N_{2} A_{\rho}^{2} N_{3} B_{\mu \nu}^{3}-\frac{1}{2} \eta_{\rho}^{2} N_{3} B_{\mu \nu}^{3}-2 \eta_{\mu \nu}^{3} N_{2} A_{\rho}^{2}\right)+\frac{\rho_{2}}{2}\left(\eta_{\rho}^{2}-N_{2} A_{\rho}^{2}\right)^{2}-\frac{i}{4 \pi} \varepsilon^{\rho \lambda \mu \nu} \eta_{\rho}^{2} \partial_{\lambda} B_{\mu \nu}^{2} \\
& +i \Lambda \varepsilon^{\mu \nu \lambda \rho} \eta_{\rho}^{2}\left(N_{3} B_{\mu \nu}^{3} N_{1} A_{\lambda}^{1}-\frac{1}{2} \eta_{\lambda}^{1} N_{3} B_{\mu \nu}^{3}-2 \eta_{\mu \nu}^{3} N_{1} A_{\lambda}^{1}\right)-i N_{1} N_{2} N_{3} \Lambda \varepsilon^{\mu \nu \lambda \rho} A_{\lambda}^{1} A_{\rho}^{2} B_{\mu \nu}^{3}+\mathcal{L}_{\text {Maxwell }} \\
= & -\frac{i}{2 \pi} \varepsilon^{\mu \nu \lambda \rho} \eta_{\mu \nu}^{3} \partial_{\lambda} A_{\rho}^{3}-\frac{i}{4 \pi} \varepsilon^{\lambda \rho \mu \nu} \eta_{\lambda}^{1} \partial_{\rho} B_{\mu \nu}^{1}-\frac{i}{4 \pi} \varepsilon^{\rho \lambda \mu \nu} \eta_{\rho}^{2} \partial_{\lambda} B_{\mu \nu}^{2}+\frac{\rho_{3}}{2}\left(2 \eta_{\mu \nu}^{3}-N_{3} B_{\mu \nu}^{3}\right)^{2}+\frac{\rho_{1}}{2}\left(\eta_{\lambda}^{1}-N_{1} A_{\lambda}^{1}\right)^{2} \\
& +\frac{\rho_{2}}{2}\left(\eta_{\rho}^{2}-N_{2} A_{\rho}^{2}\right)^{2}+i \Lambda \varepsilon^{\mu \nu \lambda \rho}\left[2\left(\eta_{\lambda}^{1}-N_{1} A_{\lambda}^{1}\right)\left(\eta_{\rho}^{2}-N_{2} A_{\rho}^{2}\right)\left(\eta_{\mu \nu}^{3}-\frac{1}{2} N_{3} B_{\mu \nu}^{3}\right)-2 N_{1} N_{2} N_{3} \eta_{\lambda}^{1} \eta_{\rho}^{2} \eta_{\mu \nu}^{3}\right]+\mathcal{L}_{\text {Maxwell }} .
\end{aligned}
$$

Since we consider the vortexline condensation for $\rho_{3}$ and particle condensation for $\rho_{1}$ and $\rho_{2}$, i.e., $\rho_{3} \rightarrow \infty, \rho_{1} \rightarrow \infty$ and $\rho_{2} \rightarrow \infty$ are taken. These limit conditions enforce that $\eta_{\lambda}^{1}=N_{1} A_{\lambda}^{1}, \eta_{\rho}^{2}=N_{2} A_{\rho}^{2}$ and $\eta_{\mu \nu}^{3}=\frac{1}{2} N_{3} B_{\mu \nu}^{3}$. So we obtain

$$
\begin{aligned}
\mathcal{L} & =-\frac{i}{2 \pi} \varepsilon^{\mu \nu \lambda \rho} \frac{1}{2} N_{3} B_{\mu \nu}^{3} \partial_{\lambda} A_{\rho}^{3}-\frac{i}{4 \pi} \varepsilon^{\lambda \rho \mu \nu} N_{1} A_{\lambda}^{1} \partial_{\rho} B_{\mu \nu}^{1}-\frac{i}{4 \pi} \varepsilon^{\rho \lambda \mu \nu} N_{2} A_{\rho}^{2} \partial_{\lambda} B_{\mu \nu}^{2}-i N_{1} N_{2} N_{3} \Lambda \varepsilon^{\mu \nu \lambda \rho} A_{\lambda}^{1} A_{\rho}^{2} B_{\mu \nu}^{3}+\mathcal{L}_{\text {Maxwell }} \\
& =-\frac{i N_{3}}{4 \pi} \varepsilon^{\mu \nu \lambda \rho} B_{\mu \nu}^{3} \partial_{\lambda} A_{\rho}^{3}+\frac{i N_{1}}{4 \pi} \varepsilon^{\lambda \rho \mu \nu} B_{\mu \nu}^{1} \partial_{\rho} A_{\lambda}^{1}+\frac{i N_{2}}{4 \pi} \varepsilon^{\rho \lambda \mu \nu} B_{\mu \nu}^{2} \partial_{\lambda} A_{\rho}^{2}-i N_{1} N_{2} N_{3} \Lambda \varepsilon^{\mu \nu \lambda \rho} A_{\lambda}^{1} A_{\rho}^{2} B_{\mu \nu}^{3}+\mathcal{L}_{\text {Maxwell }} \\
& =-\frac{i N_{3}}{4 \pi} \varepsilon^{\mu \nu \lambda \rho} B_{\mu \nu}^{3} \partial_{\lambda} A_{\rho}^{3}-\frac{i N_{1}}{4 \pi} \varepsilon^{\mu \nu \lambda \rho} B_{\mu \nu}^{1} \partial_{\lambda} A_{\rho}^{1}-\frac{i N_{2}}{4 \pi} \varepsilon^{\mu \nu \lambda \rho} B_{\mu \nu}^{2} \partial_{\lambda} A_{\rho}^{2}-i N_{1} N_{2} N_{3} \Lambda \varepsilon^{\mu \nu \lambda \rho} A_{\mu}^{1} A_{\nu}^{2} B_{\lambda \rho}^{3}+\mathcal{L}_{\text {Maxwell }} .
\end{aligned}
$$

The Maxwell kinetic term $\mathcal{L}_{\text {Maxwell }}$ can be neglected since its scaling dimension is more irrelevant than the other topological terms. Finally, we can drop the overall minus sign by relabeling indices and then obtain

$$
\mathcal{L}=\frac{i N_{1}}{4 \pi} \varepsilon^{\mu \nu \lambda \rho} B_{\mu \nu}^{1} \partial_{\lambda} A_{\rho}^{1}+\frac{i N_{2}}{4 \pi} \varepsilon^{\mu \nu \lambda \rho} B_{\mu \nu}^{2} \partial_{\lambda} A_{\rho}^{2}+\frac{i N_{3}}{4 \pi} \varepsilon^{\mu \nu \lambda \rho} B_{\mu \nu}^{3} \partial_{\lambda} A_{\rho}^{3}+i N_{1} N_{2} N_{3} \Lambda \varepsilon^{\mu \nu \lambda \rho} A_{\mu}^{1} A_{\nu}^{2} B_{\lambda \rho}^{3} .
$$

\section{APPENDIX B: ALL LEGITIMATE TQFT ACTIONS WHEN $G=\prod_{i=1}^{4} \mathbb{Z}_{N_{i}}$}

The general expression of legitimate TQFT actions and corresponding gauge transformations are listed in Table IV. By properly reassigning the indices $\{i, j, k, l\}$ (see the captions in Table IV), we can obtain all possible legitimate TQFT actions. As discussed in Sec. III C, when $G=\prod_{i=1}^{4} \mathbb{Z}_{N_{i}}$, the legitimate TQFT actions can be classified by the properties of BR braidings they describe:

(1) $S$ with no $A A B$ terms. The set of compatible braiding phases is

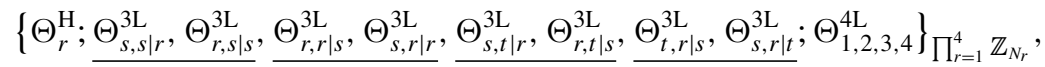

where $r<s<t$ and $\{r, s, t\} \subset\{1,2,3,4\}$. The underlines denote the linear dependence between braiding phases: $\Theta_{s, s \mid r}^{3 \mathrm{~L}}=$ $-2 \cdot \Theta_{r, s \mid s}^{3 \mathrm{~L}}, \Theta_{s, t \mid r}^{3 \mathrm{~L}}=-\Theta_{r, t \mid s}^{3 \mathrm{~L}}$, etc.

(2) $S$ with $A A B$ terms which involve one flavor of $B^{i}$. The set of compatible braiding phases is

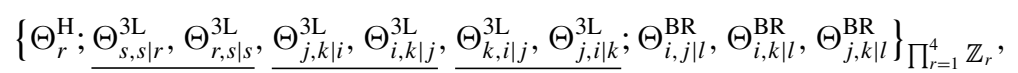

where $r \neq s, r \neq l, s \neq l$, and the assignments for $(i, j, k, l)$ are $(1,2,3,4),(2,3,4,1),(3,4,1,2)$, and $(4,1,2,3)$.

(3) $S$ with $A A B$ terms which involve two flavors of $B^{i}$. The set of compatible braiding phases is

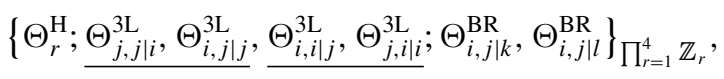

where the assignments for $(i, j, k, l)$ are $(3,4,1,2),(2,4,1,3),(2,3,1,4),(4,1,2,3),(3,1,2,4)$, and $(1,2,3,4)$.

\section{APPENDIX C: SOME TECHNICAL DETAILS}

\section{Derivation for the incompatibility of $A^{1} A^{2} B^{3}$ and $A^{2} A^{3} B^{1}$}

We try to find proper gauge transformations for this action:

$$
S=\int \sum_{i=1}^{3} \frac{N_{i}}{2 \pi} B^{i} d A^{i}+\frac{p_{12,3}}{(2 \pi)^{2}} A^{1} A^{2} B^{3}+\frac{p_{23,1}}{(2 \pi)^{2}} A^{2} A^{3} B^{1} .
$$


TABLE V. Test gauge transformations with $A^{1} \rightarrow A^{1}+d \chi^{1}$ for $S=\int \sum_{i=1}^{3} \frac{N_{i}}{2 \pi} B^{i} d A^{i}+\frac{p_{12,3}}{(2 \pi)^{2}} A^{1} A^{2} B^{3}+\frac{p_{23,1}}{(2 \pi)^{2}} A^{2} A^{3} B^{1}$. The stubborn terms are terms which cannot be eliminated by subtraction nor be absorbed into a total derivative term, making $\Delta S \neq 0 \bmod 2 \pi$.

\begin{tabular}{|c|c|c|}
\hline Test gauge transformations & $\Delta S$ & Stubborn term \\
\hline $\begin{array}{l}A^{1} \rightarrow A^{1}+d \chi^{1} \\
A^{2} \rightarrow A^{2}+d \chi^{2} \\
A^{3} \rightarrow A^{3}+d \chi^{3}+X^{3} \\
B^{1} \rightarrow B^{1}+d V^{1}+Y^{1} \\
B^{2} \rightarrow B^{2}+d V^{2}+Y^{2} \\
B^{3} \rightarrow B^{3}+d V^{3}\end{array}$ & $\begin{array}{l}\int \sum_{i=1}^{3} \frac{N_{i}}{2 \pi} Y^{i} d A^{i}+\frac{p_{12,3}}{(2 \pi)^{2}}\left(d \chi^{1} A^{2} B^{3}+A^{1} d \chi^{2} B^{3}+d \chi^{1} d \chi^{2} B^{3}\right) \\
\quad+\frac{p_{12,3}}{(2 \pi)^{2}}\left(A^{1} A^{2} d V^{3}+d \chi^{1} A^{2} d V^{3}+A^{1} d \chi^{2} d V^{3}+d \chi^{1} d \chi^{2} d V^{3}\right) \\
\quad+\frac{p_{12,3}}{(2 \pi)^{2}}\left(A^{1} A^{2} Y^{3}+d \chi^{1} A^{2} Y^{3}+A^{1} d \chi^{2} Y^{3}+d \chi^{1} d \chi^{2} Y^{3}\right) \\
\quad+\frac{p_{23,1}}{(2 \pi)^{2}}\left(d \chi^{2} A^{3} B^{1}+A^{2} d \chi^{3} B^{1}+d \chi^{2} d \chi^{3} B^{1}\right) \\
\quad+\frac{p_{23,1}}{(2 \pi)^{2}}\left(A^{2} A^{3} d V^{1}+d \chi^{2} A^{3} d V^{1}+A^{2} d \chi^{3} d V^{1}+d \chi^{2} d \chi^{3} d V^{1}\right) \\
\quad+\frac{p_{23,1}}{(2 \pi)^{2}}\left(A^{2} A^{3} Y^{1}+d \chi^{2} A^{3} Y^{1}+A^{2} d \chi^{3} Y^{1}+d \chi^{2} d \chi^{3} Y^{1}\right) \\
\int \sum_{i=1}^{2} \frac{N_{i}}{2 \pi} Y^{i} d A^{i}+\frac{N_{3}}{2 \pi}\left(d V^{3} d A^{3}+B^{3} d X^{3}+d V^{3} d X^{3}\right) \\
\quad+\frac{p_{12,3}}{(2 \pi)^{2}}\left(d \chi^{1} A^{2} B^{3}+A^{1} d \chi^{1} B^{3}+d \chi^{1} d \chi^{2} B^{3}\right) \\
\quad+\frac{p_{12,3}}{(2 \pi)^{2}}\left(A^{1} A^{2} d V^{3}+d \chi^{1} A^{2} d V^{3}+A^{1} d \chi^{1} d V^{3}+d \chi^{1} d \chi^{2} d V^{3}\right) \\
\quad+\frac{p_{23,1}}{(2 \pi)^{2}}\left(d \chi^{2} A^{3} B^{1}+A^{2} d \chi^{3} B^{1}+d \chi^{2} d \chi^{3} B^{1}\right) \\
\quad+\frac{p_{23,1}}{(2 \pi)^{2}}\left(A^{2} A^{3} d V^{1}+d \chi^{2} A^{3} d V^{1}+A^{2} d \chi^{3} d V^{1}+d \chi^{2} d \chi^{3} d V^{1}\right) \\
\quad+\frac{p_{23,1}}{(2 \pi)^{2}}\left(A^{2} A^{3} Y^{1}+d \chi^{2} A^{3} Y^{1}+A^{2} d \chi^{3} Y^{1}+d \chi^{2} d \chi^{3} Y^{1}\right)\end{array}$ & $\begin{array}{l}d \chi^{1} d \chi^{2} B^{3}, \\
d \chi^{2} d \chi^{3} B^{1}\end{array}$ \\
\hline
\end{tabular}

We list all four possible test gauge transformations respecting the $\mathbb{Z}_{N_{1}}$ and $\mathbb{Z}_{N_{3}}$ cyclic group structure and corresponding $\Delta S$ 's in Tables $\mathrm{V}$ and VI. We point out that there always exist stubborn terms which cannot be eliminated by subtraction nor be absorbed into a total derivative term, making $\Delta S$ nonvanishing. If we impose that $\Delta S=0$ mod $2 \pi$, the $\mathbb{Z}_{N_{1}}$ and $\mathbb{Z}_{N_{3}}$ cyclic group structures are no longer respected. Such a dilemma indicates that Eq. (C1) is not a legitimate TQFT action, thus $A^{1} A^{2} B^{3}$ and $A^{2} A^{3} B^{1}$ are incompatible.

For the second column of Table VI, it may not be so straightforward to see that $\Delta S$ is nonvanishing. Below we make some illustration. The gauge transformations are

$$
\begin{aligned}
& A^{1} \rightarrow A^{1}+d \chi^{1}+X^{1}, \\
& A^{2} \rightarrow A^{2}+d \chi^{2}, \\
& A^{3} \rightarrow A^{3}+d \chi^{3}+X^{3}, \\
& B^{1} \rightarrow B^{1}+d V^{1}, \\
& B^{2} \rightarrow B^{2}+d V^{2}+Y^{2}, \\
& B^{3} \rightarrow B^{3}+d V^{3} .
\end{aligned}
$$

TABLE VI. Test gauge transformations with $B^{1} \rightarrow B^{1}+d V^{1}$ for $S=\int \sum_{i=1}^{3} \frac{N_{i}}{2 \pi} B^{i} d A^{i}+\frac{p_{12,3}}{(2 \pi)^{2}} A^{1} A^{2} B^{3}+\frac{p_{23,1}}{(2)^{2}} A^{2} A^{3} B^{1}$. The stubborn terms are terms which cannot be eliminated by subtraction nor be absorbed into a total derivative term, making $\Delta S \not 0 \bmod 2 \pi$.

Test gauge transformations

$\Delta S$

Stubborn terms

$$
\begin{aligned}
& A^{1} \rightarrow A^{1}+d \chi^{1}+X^{1} \\
& A^{2} \rightarrow A^{2}+d \chi^{2} \\
& A^{3} \rightarrow A^{3}+d \chi^{3} \\
& B^{1} \rightarrow B^{1}+d V^{1} \\
& B^{2} \rightarrow B^{2}+d V^{2}+Y^{2} \\
& B^{3} \rightarrow B^{3}+d V^{3}+Y^{3}
\end{aligned}
$$$$
A^{1} \rightarrow A^{1}+d \chi^{1}+X^{1}
$$$$
A^{2} \rightarrow A^{2}+d \chi^{2}
$$$$
A^{3} \rightarrow A^{3}+d \chi^{3}+X^{3}
$$$$
B^{1} \rightarrow B^{1}+d V^{1}
$$$$
B^{2} \rightarrow B^{2}+d V^{2}+Y^{2}
$$$$
B^{3} \rightarrow B^{3}+d V^{3}
$$

$$
\begin{aligned}
& \int \frac{N_{1}}{2 \pi} d V^{1} d A^{1}+\frac{N_{1}}{2 \pi} B^{1} d X^{1}+\frac{N_{1}}{2 \pi} d V^{1} d X^{1}+\sum_{i=2}^{3} \frac{N_{i}}{2 \pi} Y^{i} d A^{i} \\
& +\frac{p_{12,3}}{(2 \pi)^{2}}\left(A^{1} d \chi^{2} B^{3}+d \chi^{1} A^{2} B^{3}+d \chi^{1} d \chi^{2} B^{3}+X^{1} A^{2} B^{3}+X^{1} d \chi^{2} B^{3}\right) \\
& +\frac{p_{12,3}}{(2 \pi)^{2}}\left(A^{1} A^{2} d V^{3}+A^{1} d \chi^{2} d V^{3}+d \chi^{1} A^{2} d V^{3}+d \chi^{1} d \chi^{2} d V^{3}+X^{1} A^{2} d V^{3}+X^{1} d \chi^{2} d V^{3}\right) \\
& +\frac{p_{12,3}}{(2 \pi)^{2}}\left(A^{1} A^{2} Y^{3}+A^{1} d \chi^{2} Y^{3}+d \chi^{1} A^{2} Y^{3}+d \chi^{1} d \chi^{2} Y^{3}+X^{1} A^{2} Y^{3}+X^{1} d \chi^{2} Y^{3}\right) \\
& d \chi^{1} d \chi^{2} B^{3} \\
& +\frac{p_{23,1}}{(2 \pi)^{2}}\left(A^{2} A^{3}+d \chi^{2} A^{3} B^{1}+A^{2} d \chi^{3} B^{1}+d \chi^{2} d \chi^{3} B^{1}\right) \\
& +\frac{p_{23,1}}{(2 \pi)^{2}}\left(A^{2} A^{3} d V^{1}+d \chi^{2} A^{3} d V^{1}+A^{2} d \chi^{3} d V^{1}+d \chi^{2} d \chi^{3} d V^{1}\right) \\
& +\frac{p_{23,1}}{(2 \pi)^{2}}\left(A^{2} A^{3} Y^{1}+d \chi^{2} A^{3} Y^{1}+A^{2} d \chi^{3} Y^{1}+d \chi^{2} d \chi^{3} Y^{1}\right) \\
& \int \frac{N_{1}}{2 \pi} B^{1} d X^{1}+\frac{N_{1}}{2 \pi} d V^{1} d X^{1}+\frac{N_{2}}{2 \pi} Y^{2} d A^{2}+\frac{N_{3}}{2 \pi} B^{3} d X^{3}+\frac{N_{3}}{2 \pi} d V^{3} d X^{3} \\
& +\frac{p_{12,3}}{(2 \pi)^{2}}\left(A^{1} d \chi^{2} B^{3}+d \chi^{1} A^{2} B^{3}+d \chi^{1} d \chi^{2} B^{3}+X^{1} A^{2} B^{3}+X^{1} d \chi^{2} B^{3}\right) \\
& +\frac{p_{12,3}}{(2 \pi)^{2}}\left(A^{1} A^{2} d V^{3}+A^{1} d \chi^{2} d V^{3}+d \chi^{1} A^{2} d V^{3}+d \chi^{1} d \chi^{2} d V^{3}+X^{1} A^{2} d V^{3}+X^{1} d \chi^{2} d V^{3}\right) \quad A^{1} A^{2} d V^{3}, \\
& +\frac{p_{23,1}}{(2 \pi)^{2}}\left(d \chi^{2} A^{3} B^{1}+A^{2} d \chi^{3} B^{1}+d \chi^{2} d \chi^{3} B^{1}+A^{2} X^{3} B^{1}+d \chi^{2} X^{3} B^{1}\right) \\
& A^{2} A^{3} d V^{1} \\
& +\frac{p_{23,1}}{(2 \pi)^{2}}\left(A^{2} A^{3} d V^{1}+d \chi^{2} A^{3} d V^{1}+A^{2} d \chi^{3} d V^{1}+d \chi^{2} d \chi^{3} d V^{1}+A^{2} X^{3} d V^{1}+d \chi^{2} X^{3} d V^{1}\right)
\end{aligned}
$$


The variation of action (boundary terms neglected) is

$$
\begin{aligned}
\Delta S= & \int \frac{N_{1}}{2 \pi} B^{1} d X^{1}+\frac{N_{2}}{2 \pi} Y^{2} d A^{2}+\frac{N_{3}}{2 \pi} B^{3} d X^{3}+\frac{p_{12,3}}{(2 \pi)^{2}}\left(A^{1} d \chi^{2} B^{3}+d \chi^{1} A^{2} B^{3}+d \chi^{1} d \chi^{2} B^{3}+X^{1} A^{2} B^{3}+X^{1} d \chi^{2} B^{3}\right) \\
& +\frac{p_{12,3}}{(2 \pi)^{2}}\left(A^{1} A^{2} d V^{3}+A^{1} d \chi^{2} d V^{3}+d \chi^{1} A^{2} d V^{3}+X^{1} A^{2} d V^{3}+X^{1} d \chi^{2} d V^{3}\right) \\
& +\frac{p_{23,1}}{(2 \pi)^{2}}\left(d \chi^{2} A^{3} B^{1}+A^{2} d \chi^{3} B^{1}+d \chi^{2} d \chi^{3} B^{1}+A^{2} X^{3} B^{1}+d \chi^{2} X^{3} B^{1}\right) \\
& +\frac{p_{23,1}}{(2 \pi)^{2}}\left(A^{2} A^{3} d V^{1}+d \chi^{2} A^{3} d V^{1}+A^{2} d \chi^{3} d V^{1}+A^{2} X^{3} d V^{1}+d \chi^{2} X^{3} d V^{1}\right) .
\end{aligned}
$$

If we want to eliminate the $A^{1} A^{2} d V^{3}$ term by subtraction, we can only require that

$$
\begin{aligned}
\frac{N_{3}}{2 \pi} d V^{3} d X^{3}+\frac{p_{12,3}}{(2 \pi)^{2}} A^{1} A^{2} d V^{3} & =\frac{p_{12,3}}{(2 \pi)^{2}}\left[d V^{3}\left(\frac{N_{3}}{2 \pi} \cdot \frac{(2 \pi)^{2}}{p_{12,3}} d X^{3}+A^{1} A^{2}\right)\right] \\
& =\frac{p_{12,3}}{(2 \pi)^{2}}\left[d V^{3}\left(\frac{N_{3}}{2 \pi} \cdot \frac{(2 \pi)^{2}}{p_{12,3}} \cdot\left(-A^{1} A^{2}+\cdots\right)+A^{1} A^{2}\right)\right] \\
& =\frac{N_{3}}{2 \pi} d V^{3}(\cdots),
\end{aligned}
$$

which requires

$$
d X^{3}=-A^{1} A^{2}+\cdots .
$$

However, Eq. (C5) is impossible since $A^{1} A^{2}$ is not exact in general. If we want to absorb $A^{1} A^{2} d V^{3}$ into a total derivative term, we would need a $\left(d A^{1} A^{2} V^{3}-A^{1} d A^{2} V^{3}\right)$ term since $d\left(A^{1} A^{2} V^{3}\right)=d A^{1} A^{2} V^{3}-A^{1} d A^{2} V^{3}+A^{1} A^{2} d V^{3}$. We may have a $-A^{1} d A^{2} V^{3}$ term contributed by $\frac{N_{2}}{2 \pi} Y^{2} d A^{2}$, but we do not have a $d A^{1} A^{2} V^{3}$ term since no term containing $d A^{1}$ in $\Delta S$. Therefore, action $S=\int \sum_{i=1}^{3} \frac{N_{i}}{2 \pi} B^{i} d A^{i}+\frac{p_{12,3}}{(2 \pi)^{2}} A^{1} A^{2} B^{3}+\frac{p_{23,1}}{(2 \pi)^{2}} A^{2} A^{3} B^{1}$ is not gauge invariant under Eqs. (C2).

\section{Derivation of the incompatibility between $A^{1} A^{3} d A^{3}$ and $A^{1} A^{2} B^{3}$}

The TQFT action is assumed to be

$$
S=\int \sum_{i=1}^{3} \frac{N_{i}}{2 \pi} B^{i} d A^{i}+\frac{q_{133}}{(2 \pi)^{2}} A^{1} A^{3} d A^{3}+\frac{p_{12,3}}{(2 \pi)^{2}} A^{1} A^{2} B^{3} .
$$

First, we assume that the gauge transformations are

$$
\begin{aligned}
& A^{i} \rightarrow A^{i}+d \chi^{i}, \\
& B^{i} \rightarrow B^{i}+d V^{i}+Y^{i},
\end{aligned}
$$

i.e., the $\mathbb{Z}_{N_{3}}$ cyclic group structure is encoded in

$$
\oint A^{3} \in \frac{2 \pi}{N_{3}} \mathbb{Z}_{N_{3}}
$$

Under the gauge transformations, the variation of action (boundary terms neglected) is

$$
\begin{aligned}
\Delta S= & \int \frac{N_{1}}{2 \pi} Y^{1} d A^{1}+\frac{N_{2}}{2 \pi} Y^{2} d A^{2}+\frac{N_{3}}{2 \pi} Y^{3} d A^{3} \\
& +\frac{q_{133}}{(2 \pi)^{2}}\left(d \chi^{1} A^{3} d A^{3}+A^{1} d \chi^{3} d A^{3}\right) \\
& +\frac{p_{12,3}}{(2 \pi)^{2}}\left[\left(A^{1} d \chi^{2} B^{3}+d \chi^{1} A^{2} B^{3}+d \chi^{1} d \chi^{2} B^{3}\right)\right. \\
& +\left(A^{1} A^{2} d V^{3}+A^{1} d \chi^{2} d V^{3}+d \chi^{1} A^{2} d V^{3}\right) \\
& \left.+\left(A^{1} A^{2} Y^{3}+A^{1} d \chi^{2} Y^{3}+d \chi^{1} A^{2} Y^{3}+d \chi^{1} d \chi^{2} Y^{3}\right)\right] .
\end{aligned}
$$

$\Delta S$ is expected to be boundary terms. However, we are going to prove that this is impossible: The $d \chi^{1} d \chi^{2} B^{3}$ term cannot be eliminated by subtraction nor be absorbed into a total derivative term. If $d \chi^{1} d \chi^{2} B^{3}$ can be eliminated by subtraction, it is required that

$$
d \chi^{1} d \chi^{2} B^{3}+d \chi^{1} d \chi^{2} Y^{3}=d \chi^{1} d \chi^{2}\left(B^{3}-B^{3}+\cdots\right),
$$

i.e.,

$$
Y^{3}=-B^{3}+\cdots
$$

but this is not allowed-otherwise the gauge transformation of $B^{3}$ is ill-defined. If $d \chi^{1} d \chi^{2} B^{3}$ can be absorb into a total derivative term, $\Delta S$ should (but in fact does not) contain a $-\chi^{1} d \chi^{2} d B^{3}$ term since

$$
d\left(\chi^{1} d \chi^{2} B^{3}\right)=d \chi^{1} d \chi^{2} B^{3}-\chi^{1} d \chi^{2} d B^{3} .
$$

Therefore, the action Eq. (C6) is impossible to be gauge invariant under gauge transformation Eqs. (C7), hence it is not a legitimate TQFT action.

Next, we assume that the gauge transformations are

$$
\begin{aligned}
A^{1,2} & \rightarrow A^{1,2}+d \chi^{1,2}, \\
A^{3} & \rightarrow A^{3}+d \chi^{3}+X^{3}, \\
B^{1,2} & \rightarrow B^{1,2}+d V^{1,2}+Y^{1,2}, \\
B^{3} & \rightarrow B^{3}+d V^{3} .
\end{aligned}
$$


The $\mathbb{Z}_{N_{3}}$ cyclic group structure is encoded in

$$
\oint B^{3} \in \frac{2 \pi}{N_{3}} \mathbb{Z}_{N_{3}} \text {. }
$$

Under the gauge transformation, the variation of action is (boundary terms neglected) is

$$
\begin{aligned}
\Delta S= & \int \frac{N_{1}}{2 \pi} Y^{1} d A^{1}+\frac{N_{2}}{2 \pi} Y^{2} d A^{2}+\frac{N_{3}}{2 \pi} B^{3} d X^{3} \\
& +\frac{q_{133}}{(2 \pi)^{2}}\left(d \chi^{1} A^{3} d A^{3}+A^{1} d \chi^{3} d A^{3}\right) \\
& +\frac{q_{133}}{(2 \pi)^{2}}\left(A^{1} X^{3} d A^{3}+d \chi^{1} X^{3} d A^{3}\right) \\
& +\frac{q_{133}}{(2 \pi)^{2}}\left(A^{1} A^{3} d X^{3}+d \chi^{1} A^{3} d X^{3}+A^{1} d \chi^{3} d X^{3}\right) \\
& +\frac{q_{133}}{(2 \pi)^{2}}\left(A^{1} X^{3} d X^{3}+d \chi^{1} X^{3} d X^{3}\right) \\
& +\frac{p_{12,3}}{(2 \pi)^{2}}\left(d \chi^{1} A^{2} B^{3}+A^{1} d \chi^{2} B^{3}+d \chi^{1} d \chi^{2} B^{3}\right) \\
& +\frac{p_{12,3}}{(2 \pi)^{2}}\left(A^{1} A^{2} d V^{3}+d \chi^{1} A^{2} d V^{3}+A^{1} d \chi^{2} d V^{3}\right) .
\end{aligned}
$$

Similarly, $\Delta S$ is impossible to be boundary terms since the term $d \chi^{1} A^{3} d A^{3}$ cannot be eliminated by subtraction nor be absorbed into a total derivative term. The only way to eliminate $d \chi^{1} A^{3} d A^{3}$ by subtraction is to require that

$$
d \chi^{1} A^{3} d A^{3}+d \chi^{1} X^{3} d A^{3}=0,
$$

which means that

$$
X^{3}=-A^{3}+\cdots .
$$

If so, the gauge transformation of $A^{3}$ is

$$
A^{3} \rightarrow d \chi^{3}+\cdots
$$

which is not well-defined. If $d \chi^{1} A^{3} d A^{3}$ can be absorbed into a total derivative term, $\Delta S$ should (but, in fact, does not) contain a $\chi^{1} d A^{3} d A^{3}$ term since

$$
d\left(\chi^{1} A^{3} d A^{3}\right)=d \chi^{1} A^{3} d A^{3}+\chi^{1} d A^{3} d A^{3},
$$

Therefore, Eqs. (C13) cannot be the proper gauge transformations for Eq. (52).

So far, we have seen that if the $\mathbb{Z}_{N_{3}}$ cyclic group structure is respected, we cannot find a proper set of gauge transformations under which the action Eq. (52) is invariant up to boundary terms. If the action is imposed to be gauge invariant up to boundary terms, the $\mathbb{Z}_{N_{3}}$ cyclic group structure would be violated. This dilemma reveals that the action Eq. (52) is not a legitimate TQFT theory. In other words, $A^{1} A^{3} d A^{3}$ is incompatible with $A^{1} A^{2} B^{3}$, i.e., $\Theta_{3,3 \mid 1}^{3 \mathrm{~L}}$ is incompatible with $\Theta_{1,2 \mid 3}^{\mathrm{BR}}$.

\section{Derivation of gauge transformations for $S=\int \sum_{i=1}^{3} \frac{N_{i}}{2 \pi} B^{i} d A^{i}+\frac{q_{123}}{(2 \pi)^{2}} A^{1} A^{2} d A^{3}+\frac{p_{12,3}}{(2 \pi)^{2}} A^{1} A^{2} B^{3}$}

The action is

$$
S=\int \sum_{i=1}^{3} \frac{N_{i}}{2 \pi} B^{i} d A^{i}+\frac{q_{123}}{(2 \pi)^{2}} A^{1} A^{2} d A^{3}+\frac{p_{12,3}}{(2 \pi)^{2}} A^{1} A^{2} B^{3} .
$$

First, we assume $A^{3} \rightarrow A^{3}+d \chi^{3}$. The test gauge transformations are

$$
\begin{aligned}
& A^{i} \rightarrow A^{i}+d \chi^{i}, \\
& B^{i} \rightarrow B^{i}+d V^{i}+Y^{i} .
\end{aligned}
$$

Under Eqs. (C21), the variation of action is (boundary terms neglected)

$$
\begin{aligned}
\Delta S= & \int \sum_{i=1}^{3} \frac{N_{i}}{2 \pi} Y^{i} d A^{i} \\
& +\frac{q_{123}}{(2 \pi)^{2}}\left(A^{1} d \chi^{2} d A^{3}+d \chi^{1} A^{2} d A^{3}+d \chi^{1} d \chi^{2} d A^{3}\right) \\
& +\frac{p_{12,3}}{(2 \pi)^{2}}\left[\left(d \chi^{1} A^{2} B^{3}+A^{1} d \chi^{2} B^{3}+d \chi^{1} d \chi^{2} B^{3}\right)\right. \\
& +\left(A^{1} A^{2} d V^{3}+d \chi^{1} A^{2} d V^{3}+A^{1} d \chi^{2} d V^{3}\right) \\
& \left.+\left(A^{1} A^{2} Y^{3}+d \chi^{1} A^{2} Y^{3}+A^{1} d \chi^{2} Y^{3}+d \chi^{1} d \chi^{2} Y^{3}\right)\right] .
\end{aligned}
$$

Notice that the term $d \chi^{1} d \chi^{2} B^{3}$ cannot be eliminated by subtraction nor absorbed into a total derivative term. Therefore, action Eq. (55) is not gauge invariant under transformation Eqs. (C21).

Second, we assume $B^{3} \rightarrow B^{3}+d V^{3}$. The test gauge transformations are

$$
\begin{aligned}
A^{1,2} & \rightarrow A^{1,2}+d \chi^{1,2}, \\
A^{3} & \rightarrow A^{3}+d \chi^{3}+X^{3}, \\
B^{1,2} & \rightarrow B^{1,2}+d V^{1,2}+Y^{1,2}, \\
B^{3} & \rightarrow B^{3}+d V^{3}
\end{aligned}
$$

Under Eqs. (C23), the variation of action is (boundary terms neglected)

$$
\begin{aligned}
\Delta S= & \int \frac{N_{1}}{2 \pi} Y^{1} d A^{1}+\frac{N_{2}}{2 \pi} Y^{2} d A^{2}+\frac{N_{3}}{2 \pi} B^{3} d X^{3}+\frac{q_{123}}{(2 \pi)^{2}}(\underbrace{A^{1} d \chi^{2} d A^{3}+d \chi^{1} A^{2} d A^{3}+d \chi^{1} d \chi^{2} d A^{3}}_{N}) \\
& +\underbrace{\frac{q_{123}}{(2 \pi)^{2}}\left(A^{1} A^{2} d X^{3}+A^{1} d \chi^{2} d X^{3}+d \chi^{1} A^{2} d X^{3}+d \chi^{1} d \chi^{2} d X^{3}\right)}_{M} \\
& +\frac{p_{12,3}}{(2 \pi)^{2}}(\underbrace{d \chi^{1} A^{2} B^{3}+A^{1} d \chi^{2} B^{3}+d \chi^{1} d \chi^{2} B^{3}}_{O})+\frac{p_{12,3}}{(2 \pi)^{2}}(\underbrace{A^{1} A^{2} d V^{3}+d \chi^{1} A^{2} d V^{3}+A^{1} d \chi^{2} d V^{3}}_{P}) .
\end{aligned}
$$


We expect that $\Delta S$ is an integral of total derivative terms. For this purpose, we need to properly construct the shift terms, i.e., $X^{3}, Y^{1}$, and $Y^{2}$, such that $\Delta S$ is zero up to boundary terms.

For the $M$ term, notice that

$$
\begin{aligned}
& A^{1} d \chi^{2} d A^{3}+d \chi^{1} A^{2} d A^{3}+d \chi^{2} A^{3} d A^{1}-d \chi^{1} A^{3} d A^{2} \\
& =d \chi^{2} d\left(A^{1} A^{3}\right)+d \chi^{1} d\left(A^{3} A^{2}\right),
\end{aligned}
$$

thus we can let

$$
Y^{1}=\cdots+\frac{q_{123}}{(2 \pi)^{2}} \frac{2 \pi}{N_{1}} d \chi^{2} A^{3}+\cdots
$$

and

$$
Y^{2}=\cdots+\frac{q_{123}}{(2 \pi)^{2}} \frac{2 \pi}{N_{2}}\left(-d \chi^{1} A^{3}\right)+\cdots
$$

to construct the total derivative terms in Eq. (C25).

For the $O$ term, it cannot be absorbed into a total derivative term since there is no term containing $d B^{3}$ in $\Delta S$. Therefore, we have to eliminate the $O$ term by subtraction. To do this, one can assume that

$$
\begin{aligned}
& \frac{N_{3}}{2 \pi} B^{3} d X^{3}+\frac{p_{12,3}}{(2 \pi)^{2}}\left(d \chi^{1} A^{2} B^{3}+A^{1} d \chi^{2} B^{3}+d \chi^{1} d \chi^{2} B^{3}\right) \\
& \quad=0
\end{aligned}
$$

which leads to

$$
d X^{3}=\frac{p_{12,3}}{(2 \pi)^{2}} \frac{2 \pi}{N_{3}}\left(-d \chi^{1} A^{2}-A^{1} d \chi^{2}-d \chi^{1} d \chi^{2}\right) .
$$

However, we cannot find a $X^{3}$ satisfying Eq. (C29). Alternatively, we can add terms containing $d A^{1}$ or $d A^{2}$. We can let

$$
X=\frac{p_{12,3}}{(2 \pi)^{2}} \frac{2 \pi}{N_{3}}\left(-\chi^{1} A^{2}+A^{1} \chi^{2}-\chi^{1} d \chi^{2}\right),
$$

hence

$$
\begin{aligned}
d X^{3}= & \frac{p_{12,3}}{(2 \pi)^{2}} \frac{2 \pi}{N_{3}}\left(-d \chi^{1} A^{2}-\chi^{1} d A^{2}+d A^{1} \chi^{2}\right. \\
& \left.-A^{1} d \chi^{2}-d \chi^{1} d \chi^{2}\right) .
\end{aligned}
$$

Then we notice that

$$
\begin{aligned}
0= & \frac{N_{3}}{2 \pi} B^{3} d X^{3}+\frac{p_{12,3}}{(2 \pi)^{2}}\left(d \chi^{1} A^{2} B^{3}+A^{1} d \chi^{2} B^{3}+d \chi^{1} d \chi^{2} B^{3}\right) \\
& +\frac{p_{12,3}}{(2 \pi)^{2}}\left(\chi^{1} B^{3} d A^{2}-\chi^{2} B^{3} d A^{1}\right) .
\end{aligned}
$$

Therefore, we can let

$$
Y^{1}=\cdots+\frac{p_{12,3}}{(2 \pi)^{2}} \frac{2 \pi}{N_{1}}\left(-\chi^{2} B^{3}\right)+\cdots
$$

and

$$
Y^{2}=\cdots+\frac{p_{12,3}}{(2 \pi)^{2}} \frac{2 \pi}{N_{2}}\left(\chi^{1} B^{3}\right)+\cdots
$$

to contribute the $\frac{p_{12,3}}{(2 \pi)^{2}}\left(\chi^{1} B^{3} d A^{2}-\chi^{2} B^{3} d A^{1}\right)$ term in Eq. (C32).

The $N$ term, with $d X^{3}$ known in Eq. (C31), is (the underlines and underbraces are used to identify terms between equal signs)

$$
\begin{aligned}
N= & \frac{q_{123}}{(2 \pi)^{2}}\left(A^{1} A^{2}+A^{1} d \chi^{2}+d \chi^{1} A^{2}+d \chi^{1} d \chi^{2}\right) d X^{3} \\
= & \frac{q_{123}}{(2 \pi)^{2}} \frac{p_{12,3}}{(2 \pi)^{2}} \frac{2 \pi}{N_{3}}(\underbrace{A^{1} A^{2}}_{1 s t}+\underbrace{A^{1} d \chi^{2}}_{2 n d}+\underbrace{d \chi^{1} A^{2}}_{3 r d}+\underbrace{d \chi^{1} d \chi^{2}}_{4 t h})\left(-A^{1} d \chi^{2}-d \chi^{1} A^{2}-\chi^{1} d A^{2}+\chi^{2} d A^{1}-d \chi^{1} d \chi^{2}\right) \\
= & \frac{q_{123}}{(2 \pi)^{2}} \frac{p_{12,3}}{(2 \pi)^{2}} \frac{2 \pi}{N_{3}}\left[(\underbrace{-A^{1} A^{2} \chi^{1} d A^{2}+A^{1} A^{2} \chi^{2} d A^{1}-A^{1} A^{2} d \chi^{1} d \chi^{2}}_{1 s t})+(\underbrace{-A^{1} d \chi^{2} d \chi^{1} A^{2}-A^{1} d \chi^{2} \chi^{1} d A^{2}+A^{1} d \chi^{2} \chi^{2} d A^{1}}_{3 r d})\right. \\
& \left.+(\underbrace{-d \chi^{1} A^{2} A^{1} d \chi^{2}-d \chi^{1} A^{2} \chi^{1} d A^{2}+d \chi^{1} A^{2} \chi^{2} d A^{1}}_{4 \chi^{2}})+(\underbrace{-\underbrace{}_{12}}_{-d \chi^{1} d \chi^{2} \chi^{1} d A^{2}+d \chi^{1} d \chi^{2} \chi^{2} d A^{1}})\right] \\
= & \frac{q_{123}}{(2 \pi)^{2}} \frac{p_{12,3}}{(2 \pi)^{2}} \frac{2 \pi}{N_{3}}\left[-A^{1} d \chi^{2} d \chi^{1} A^{2}+\left(A^{1} A^{2} \chi^{2}+A^{1} d \chi^{2} \chi^{2}+d \chi^{1} A^{2} \chi^{2}+d \chi^{1} d \chi^{2} \chi^{2}\right) d A^{1}\right. \\
& +\underline{\left.\left(-A^{1} A^{2} \chi^{1}-A^{1} d \chi^{2} \chi^{1}-d \chi^{1} A^{2} \chi^{1}-d \chi^{1} d \chi^{2} \chi^{1}\right) d A^{2}\right] .}
\end{aligned}
$$

Notice that

$$
-A^{1} d \chi^{2} d \chi^{1} A^{2}=A^{1} A^{2} d \chi^{1} d \chi^{2}
$$

and

$$
\begin{aligned}
A^{1} A^{2} d \chi^{1} d \chi^{2}+\chi^{1} A^{2} d \chi^{2} d A^{1}-d\left(A^{1} \chi^{1}\right) \chi^{2} d A^{2} & =-A^{1} d \chi^{1} A^{2} d \chi^{2}+d A^{1} \chi^{1} A^{2} d \chi^{2}-d\left(A^{1} \chi^{1}\right) \chi^{2} d A^{2} \\
& =d\left(A^{1} \chi^{1}\right) A^{2} d \chi^{2}-d\left(A^{1} \chi^{1}\right) \chi^{2} d A^{2} \\
& =-d\left(A^{1} \chi^{1}\right) d\left(A^{2} \chi^{2}\right)
\end{aligned}
$$


we could have $\frac{N_{1}}{2 \pi} Y^{1} d A^{1}+\frac{N_{2}}{2 \pi} Y^{2} d A^{2}+$ term $N=$ total derivative terms, if

$$
Y^{1}=\cdots+\frac{q_{123}}{(2 \pi)^{2}} \frac{p_{12,3}}{(2 \pi)^{2}} \frac{2 \pi}{N_{3}} \frac{2 \pi}{N_{1}}\left[\chi^{1} A^{2} d \chi^{2}-\left(A^{1} A^{2} \chi^{2}+A^{1} d \chi^{2} \chi^{2}+d \chi^{1} A^{2} \chi^{2}+d \chi^{1} d \chi^{2} \chi^{2}\right)\right]+\cdots
$$

and

$$
Y^{2}=\cdots+\frac{q_{123}}{(2 \pi)^{2}} \frac{p_{12,3}}{(2 \pi)^{2}} \frac{2 \pi}{N_{3}} \frac{2 \pi}{N_{2}}\left[-d\left(A^{1} \chi^{1}\right) \chi^{2}+\left(A^{1} A^{2} \chi^{1}+A^{1} d \chi^{2} \chi^{1}+d \chi^{1} A^{2} \chi^{1}+d \chi^{1} d \chi^{2} \chi^{1}\right)\right]+\cdots
$$

For the $P$ term, notice that

$$
\begin{aligned}
& d\left(A^{1} A^{2} V^{3}\right)+d\left(\chi^{1} A^{2} d V^{3}\right)-d\left(A^{1} \chi^{2} d V^{3}\right) \\
& =d A^{1} A^{2} V^{3}-A^{1} d A^{2} V^{3}+\chi^{1} d A^{2} d V^{3}-d A^{1} \chi^{2} d V^{3}+\underbrace{A^{1} A^{2} d V^{3}+d \chi^{1} A^{2} d V^{3}+A^{1} d \chi^{2} d V^{3}}_{P},
\end{aligned}
$$

thus we can let

$$
Y^{1}=\cdots+\frac{p_{12,3}}{(2 \pi)^{2}} \frac{2 \pi}{N_{1}}\left(A^{2} V^{3}-\chi^{2} d V^{3}\right)+\cdots
$$

and

$$
Y^{2}=\cdots+\frac{p_{12,3}}{(2 \pi)^{2}} \frac{2 \pi}{N_{2}}\left(-A^{1} V^{3}+\chi^{1} d V^{3}\right)+\cdots
$$

to provide the $\left(d A^{1} A^{2} V^{3}-A^{1} d A^{2} V^{3}+\chi^{1} d A^{2} d V^{3}-d A^{1} \chi^{2} d V^{3}\right)$ term in Eq. (C40).

According to Eqs. (C26), (C27), (C33), (C34), (C38), (C39), (C41) and (C42), $\Delta S=\int$ (total derivative terms) or $S$ is invariant up to total derivative terms under transformation

$$
\begin{aligned}
& A^{1} \rightarrow A^{1}+d \chi^{1}, \\
& A^{2} \rightarrow A^{2}+d \chi^{2}, \\
& A^{3} \rightarrow A^{3}+d \chi^{3}+X^{3}, \\
& B^{1} \rightarrow B^{1}+d V^{1}+Y^{1}, \\
& B^{2} \rightarrow B^{2}+d V^{2}+Y^{2}, \\
& B^{3} \rightarrow B^{3}+d V^{3}
\end{aligned}
$$

where

$$
\begin{aligned}
X^{3}= & -\frac{p_{12,3}}{(2 \pi) N_{3}}\left(\chi^{1} A^{2}+\frac{1}{2} \chi^{1} d \chi^{2}\right)+\frac{p_{12,3}}{(2 \pi) N_{3}}\left(\chi^{2} A^{1}+\frac{1}{2} \chi^{2} d \chi^{1}\right), \\
Y^{1}= & \frac{q_{123}}{2 \pi N_{1}} d \chi^{2} A^{3}-\frac{p_{12,3}}{(2 \pi) N_{1}}\left(\chi^{2} B^{3}-A^{2} V^{3}+\chi^{2} d V^{3}\right) \\
& +\frac{q_{123}}{(2 \pi)^{2}} \cdot \frac{p_{12,3}}{(2 \pi) N_{3}} \cdot \frac{2 \pi}{N_{1}} \cdot\left[\chi^{1} A^{2} d \chi^{2}-\left(A^{1} A^{2} \chi^{2}+A^{1} d \chi^{2} \chi^{2}+d \chi^{1} A^{2} \chi^{2}+d \chi^{1} d \chi^{2} \chi^{2}\right)\right], \\
Y^{2}= & -\frac{q_{123}}{2 \pi N_{2}} d \chi^{1} A^{3}+\frac{p_{12,3}}{(2 \pi) N_{2}}\left(\chi^{1} B^{3}-A^{1} V^{3}+\chi^{1} d V^{3}\right) \\
& +\frac{q_{123}}{(2 \pi)^{2}} \cdot \frac{p_{12,3}}{(2 \pi) N_{3}} \cdot \frac{2 \pi}{N_{2}} \cdot\left[-d\left(A^{1} \chi^{1}\right) \chi^{2}+\left(A^{1} A^{2} \chi^{1}+A^{1} d \chi^{2} \chi^{1}+d \chi^{1} A^{2} \chi^{1}+d \chi^{1} d \chi^{2} \chi^{1}\right)\right] .
\end{aligned}
$$

\section{Derivation of incompatibility between $A^{1} A^{2} A^{3} A^{4}$ and $A^{1} A^{2} B^{4}$}

If we assume that $A^{1} A^{2} A^{3} A^{4}$ are compatible with $A^{1} A^{2} B^{4}$, the TQFT action should be

$$
S=\int \sum_{i=1}^{4} \frac{N_{i}}{2 \pi} B^{i} d A^{i}+\frac{q_{1234}}{(2 \pi)^{3}} A^{1} A^{2} A^{3} A^{4}+\frac{p_{12,4}}{(2 \pi)^{2}} A^{1} A^{2} B^{4} .
$$

In the action Eq. $(\mathrm{C} 45), B^{1}, B^{2}$, and $B^{3}$ serve as Lagrange multipliers, imposing $d A^{1}=d A^{2}=d A^{3}=0$, i.e., the gauge transformations of $A^{1}, A^{2}$, and $A^{3}$ are $A^{1,2,3} \rightarrow A^{1,2,3}+$ $d \chi^{1,2,3}$. The $\mathbb{Z}_{N_{1}}, \mathbb{Z}_{N_{2}}$, and $\mathbb{Z}_{N_{3}}$ cyclic group structures are encoded in $\oint A^{I} \in \frac{2 \pi}{N_{I}} \mathbb{Z}_{N_{I}}$, where $I=1,2,3$. The remaining $\mathbb{Z}_{N_{4}}$ cyclic group structure can be encoded in $\oint A^{4} \in \frac{2 \pi}{N_{4}} \mathbb{Z}_{N_{4}}$ or $\oint B^{4} \in \frac{2 \pi}{N_{4}} \mathbb{Z}_{N_{4}}$, corresponding to $A^{4} \rightarrow A^{4}+d \chi^{4}$ or $B^{4} \rightarrow$ $B^{4}+d V^{4}$, respectively. In the following text, we are going to examine both cases. However, we will find that neither of them would result in gauge transformations under which the action Eq. (C45) is gauge invariant up to boundary terms. 
First, we assume that the gauge transformations are

$$
\begin{aligned}
& A^{1} \rightarrow A^{1}+d \chi^{1}, \\
& A^{2} \rightarrow A^{2}+d \chi^{2}, \\
& A^{3} \rightarrow A^{3}+d \chi^{3}, \\
& A^{4} \rightarrow A^{4}+d \chi^{4}, \\
& B^{1} \rightarrow B^{1}+d V^{1}+Y^{1}, \\
& B^{2} \rightarrow B^{2}+d V^{2}+Y^{2}, \\
& B^{3} \rightarrow B^{3}+d V^{3}+Y^{3}, \\
& B^{4} \rightarrow B^{4}+d V^{4}+Y^{4} .
\end{aligned}
$$

Under Eqs. (C46), the variation of action is (boundary terms neglected)

$$
\begin{aligned}
\Delta S= & \int \sum_{i=1}^{4} \frac{N_{i}}{2 \pi} Y^{i} d A^{i}+\frac{q_{1234}}{(2 \pi)^{3}}\left(d \chi^{1} A^{2} A^{3} A^{4}+A^{1} d \chi^{2} A^{3} A^{4}+A^{1} A^{2} d \chi^{3} A^{4}+A^{1} A^{2} A^{3} d \chi^{4}\right. \\
& +d \chi^{1} d \chi^{2} A^{3} A^{4}+d \chi^{1} A^{2} d \chi^{3} A^{4}+A^{1} d \chi^{2} d \chi^{3} A^{4}+d \chi^{1} A^{2} A^{3} d \chi^{4}+A^{1} d \chi^{2} A^{3} d \chi^{4}+A^{1} A^{2} d \chi^{3} d \chi^{4} \\
& \left.+A^{1} d \chi^{2} d \chi^{3} d \chi^{4}+d \chi^{1} A^{2} d \chi^{3} d \chi^{4}+d \chi^{1} d \chi^{2} A^{3} d \chi^{4}+d \chi^{1} d \chi^{2} d \chi^{3} A^{4}+d \chi^{1} d \chi^{2} d \chi^{3} d \chi^{4}\right) \\
& +\frac{p_{12,4}}{(2 \pi)^{2}}\left(A^{1} d \chi^{2} B^{4}+d \chi^{1} A^{2} B^{4}+d \chi^{1} d \chi^{2} B^{4}\right)+\frac{p_{12,4}}{(2 \pi)^{2}}\left(A^{1} A^{2} d V^{4}+A^{1} d \chi^{2} d V^{4}+d \chi^{1} A^{2} d V^{4}+d \chi^{1} d \chi^{2} d V^{4}\right) \\
& +\frac{p_{12,4}}{(2 \pi)^{2}}\left(A^{1} A^{2} Y^{4}+A^{1} d \chi^{2} Y^{4}+d \chi^{1} A^{2} Y^{4}+d \chi^{1} d \chi^{2} Y^{4}\right) .
\end{aligned}
$$

We recognize that $d \chi^{1} d \chi^{2} B^{4}$ is a stubborn term, i.e., it cannot be eliminated by substraction or be absorbed into a total derivative term. Due to the existence of stubborn term, $\Delta S$ cannot be written as an integral of total derivative terms. Therefore, the action Eq. (C45) is not gauge invariant up to boundary terms under Eq. (C46).

Next, we assume that the gauge transformations are

$$
\begin{aligned}
& A^{1} \rightarrow A^{1}+d \chi^{1}, \\
& A^{2} \rightarrow A^{2}+d \chi^{2}, \\
& A^{3} \rightarrow A^{3}+d \chi^{3}, \\
& A^{4} \rightarrow A^{4}+d \chi^{4}+X^{4}, \\
& B^{1} \rightarrow B^{1}+d V^{1}+Y^{1}, \\
& B^{2} \rightarrow B^{2}+d V^{2}+Y^{2}, \\
& B^{3} \rightarrow B^{3}+d V^{3}+Y^{3}, \\
& B^{4} \rightarrow B^{4}+d V^{4} .
\end{aligned}
$$

Under Eq. (C46), the variation of action is (boundary terms neglected)

$$
\begin{aligned}
\Delta S= & \int \sum_{i=1}^{3} \frac{N_{i}}{2 \pi} Y^{i} d A^{i}+\frac{N_{4}}{2 \pi} B^{4} d X^{4}+\frac{q_{1234}}{(2 \pi)^{3}}\left(d \chi^{1} A^{2} A^{3} A^{4}+A^{1} d \chi^{2} A^{3} A^{4}+A^{1} A^{2} d \chi^{3} A^{4}+A^{1} A^{2} A^{3} d \chi^{4}\right. \\
& +d \chi^{1} d \chi^{2} A^{3} A^{4}+d \chi^{1} A^{2} d \chi^{3} A^{4}+A^{1} d \chi^{2} d \chi^{3} A^{4}+d \chi^{1} A^{2} A^{3} d \chi^{4}+A^{1} d \chi^{2} A^{3} d \chi^{4}+A^{1} A^{2} d \chi^{3} d \chi^{4} \\
& \left.+A^{1} d \chi^{2} d \chi^{3} d \chi^{4}+d \chi^{1} A^{2} d \chi^{3} d \chi^{4}+d \chi^{1} d \chi^{2} A^{3} d \chi^{4}+d \chi^{1} d \chi^{2} d \chi^{3} A^{4}\right) \\
& +\frac{q_{1234}}{(2 \pi)^{3}}\left(A^{1} A^{2} A^{3} X^{4}+A^{1} d \chi^{2} A^{3} X^{4}+d \chi^{1} A^{2} A^{3} X^{4}+d \chi^{1} d \chi^{2} A^{3} X^{4}\right) \\
& +\frac{q_{1234}}{(2 \pi)^{3}}\left(A^{1} A^{2} d \chi^{3} X^{4}+A^{1} d \chi^{2} d \chi^{3} X^{4}+d \chi^{1} A^{2} d \chi^{3} X^{4}+d \chi^{1} d \chi^{2} d \chi^{3} X^{4}\right) \\
& +\frac{p_{12,4}}{(2 \pi)^{2}}\left(A^{1} d \chi^{2} B^{4}+d \chi^{1} A^{2} B^{4}+d \chi^{1} d \chi^{2} B^{4}\right)+\frac{p_{12,4}}{(2 \pi)^{2}}\left(A^{1} A^{2} d V^{3}+A^{1} d \chi^{2} d V^{3}+d \chi^{1} A^{2} d V^{3}\right) .
\end{aligned}
$$


We recognize that $d \chi^{1} d \chi^{2} d \chi^{3} A^{4}$ cannot be eliminated by subtraction or be absorbed into a total derivative term. If we want to eliminate $d \chi^{1} d \chi^{2} d \chi^{3} A^{4}$ by subtraction, we could only expect that

$$
d \chi^{1} d \chi^{2} d \chi^{3} A^{4}+d \chi^{1} d \chi^{2} d \chi^{3} X^{4}=d \chi^{1} d \chi^{2} d \chi^{3}\left(A^{4}-A^{4}+\cdots\right),
$$

which means that $X^{4}=-A^{4}+\cdots$, hence the gauge transformation of $A^{4}$ is ill-defined:

$$
A^{4} \rightarrow d \chi^{4}+\cdots
$$

If we want to absorb $d \chi^{1} d \chi^{2} d \chi^{3} A^{4}$ into a total derivative term, we need a $\chi^{1} d \chi^{2} d \chi^{3} d A^{4}$ term in $\Delta S$ since

$$
d\left(\chi^{1} d \chi^{2} d \chi^{3} A^{4}\right)=d \chi^{1} d \chi^{2} d \chi^{3} A^{4}+\chi^{1} d \chi^{2} d \chi^{3} d A^{4} .
$$

However, this $\chi^{1} d \chi^{2} d \chi^{3} d A^{4}$ term does not exist in $\Delta S$. Therefore, due to the stubborn term $d \chi^{1} d \chi^{2} d \chi^{3} A^{4}$, the action Eq. (C45) is not gauge invariant up to boundary terms under Eq. (C46).

Finally, we can conclude that the action Eq. (C45) could never be gauge invariant up to boundary terms under gauge transformations which respect $\mathbb{Z}_{N_{i}}$ cyclic group structures, hence it is not a legitimate TQFT action. Therefore, $A^{1} A^{2} A^{3} A^{4}\left(\Theta_{1,2,3,4}^{4 \mathrm{~L}}\right)$ is incompatible with $A^{1} A^{2} B^{4}\left(\Theta_{1,2 \mid 4}^{\mathrm{BR}}\right)$.

[1] X.-G. Wen, Topological orders and edge excitations in fractional quantum hall states, Adv. Phys. 44, 405 (1995).

[2] X.-G. Wen, Quantum Field Theory of Many-Body Systems: From the Origin of Sound to an Origin of Light and Electrons (Oxford University Press, New York, 2004).

[3] M. A. Levin and X.-G. Wen, Colloquium: Photons and electrons as emergent phenomena, Rev. Mod. Phys. 77, 871 (2005).

[4] T. Lan, L. Kong, and X.-G. Wen, Classification of (2+1)dimensional topological order and symmetry-protected topological order for bosonic and fermionic systems with on-site symmetries, Phys. Rev. B 95, 235140 (2017).

[5] X.-G. Wen, A theory of 2+1D bosonic topological orders, Natl. Sci. Rev. 3, 68 (2016).

[6] M.-Y. Li and P. Ye, Fracton physics of spatially extended excitations, Phys. Rev. B 101, 245134 (2020).

[7] M. Levin and Z.-C. Gu, Braiding statistics approach to symmetry-protected topological phases, Phys. Rev. B 86, 115109 (2012).

[8] X. Chen, Z.-C. Gu, Z.-X. Liu, and X.-G. Wen, Symmetry protected topological orders and the group cohomology of their symmetry group, Phys. Rev. B 87, 155114 (2013).

[9] X. Chen, Z.-C. Gu, Z.-X. Liu, and X.-G. Wen, Symmetryprotected topological orders in interacting bosonic systems, Science 338, 1604 (2012).

[10] F. Pollmann, E. Berg, A. M. Turner, and M. Oshikawa, Symmetry protection of topological phases in one-dimensional quantum spin systems, Phys. Rev. B 85, 075125 (2012).

[11] Z.-C. Gu and X.-G. Wen, Tensor-entanglement-filtering renormalization approach and symmetry-protected topological order, Phys. Rev. B 80, 155131 (2009).

[12] C. Nayak, S. H. Simon, A. Stern, M. Freedman, and S. Das Sarma, Non-Abelian anyons and topological quantum computation, Rev. Mod. Phys. 80, 1083 (2008).

[13] B. Blok and X. G. Wen, Effective theories of the fractional quantum Hall effect at generic filling fractions, Phys. Rev. B 42, 8133 (1990).

[14] E. Fradkin, Field Theories of Condensed Matter Physics (Cambridge University Press, New York, 2013).

[15] L. M. Krauss and F. Wilczek, Discrete Gauge Symmetry in Continuum Theories, Phys. Rev. Lett. 62, 1221 (1989).
[16] T. H. Hansson, V. Oganesyan, and S. L. Sondhi, Superconductors are topologically ordered, Ann. Phys. 313, 497 (2004).

[17] C. Delcamp and A. Tiwari, From gauge to higher gauge models of topological phases, J. High Energy Phys. 10 (2018) 049.

[18] C. Delcamp and A. Tiwari, On 2-form gauge models of topological phases, J. High Energy Phys. 05 (2019) 064.

[19] A. Hamma, P. Zanardi, and X.-G. Wen, String and membrane condensation on three-dimensional lattices, Phys. Rev. B 72, 035307 (2005).

[20] A. Kitaev, Anyons in an exactly solved model and beyond, Ann. Phys. 321, 2 (2006).

[21] J. Preskill, Lecture Notes for Physics 219: Quantum Computation, Caltech Lecture Notes (1999), http://theory.caltech.edu/ preskill/ph219/topological.pdf.

[22] Y. Aharonov and D. Bohm, Significance of electromagnetic potentials in the quantum theory, Phys. Rev. 115, 485 (1959).

[23] J. Preskill and L. M. Krauss, Local discrete symmetry and quantum-mechanical hair, Nucl. Phys. B 341, 50 (1990).

[24] M. G. Alford and F. Wilczek, Aharonov-Bohm Interaction of Cosmic Strings with Matter, Phys. Rev. Lett. 62, 1071 (1989).

[25] M. G. Alford, K.-M. Lee, J. March-Russell, and J. Preskill, Quantum field theory of non-Abelian strings and vortices, Nucl. Phys. B 384, 251 (1992).

[26] C. Wang and M. Levin, Braiding Statistics of Loop Excitations in Three Dimensions, Phys. Rev. Lett. 113, 080403 (2014).

[27] J. C. Wang, Z.-C. Gu, and X.-G. Wen, Field-Theory Representation of Gauge-Gravity Symmetry-Protected Topological Invariants, Group Cohomology, and Beyond, Phys. Rev. Lett. 114, 031601 (2015).

[28] P. Putrov, J. Wang, and S.-T. Yau, Braiding statistics and link invariants of bosonic/fermionic topological quantum matter in 2+1 and 3+1 dimensions, Ann. Phys. 384, 254 (2017).

[29] P. Ye and Z.-C. Gu, Topological quantum field theory of threedimensional bosonic Abelian-symmetry-protected topological phases, Phys. Rev. B 93, 205157 (2016).

[30] S.-Q. Ning, Z.-X. Liu, and P. Ye, Symmetry enrichment in three-dimensional topological phases, Phys. Rev. B 94, 245120 (2016).

[31] J. C. Wang and X.-G. Wen, Non-Abelian string and particle braiding in topological order: Modular $\operatorname{SL}(3, \mathbb{Z})$ representation 
and $(3+1)$-dimensional twisted gauge theory, Phys. Rev. B 91, 035134 (2015).

[32] P. Ye, Three-dimensional anomalous twisted gauge theories with global symmetry: Implications for quantum spin liquids, Phys. Rev. B 97, 125127 (2018).

[33] S.-Q. Ning, Z.-X. Liu, and P. Ye, Topological gauge theory, symmetry fractionalization, and classification of symmetry-enriched topological phases in three dimensions, arXiv:1801.01638.

[34] Q.-R. Wang, M. Cheng, C. Wang, and Z.-C. Gu, Topological quantum field theory for Abelian topological phases and loop braiding statistics in $(3+1)$-dimensions, Phys. Rev. B 99, 235137 (2019).

[35] C.-M. Jian and X.-L. Qi, Layer Construction of 3D Topological States and String Braiding Statistics, Phys. Rev. X 4, 041043 (2014).

[36] S. Jiang, A. Mesaros, and Y. Ran, Generalized Modular Transformations in $(3+1) \mathrm{D}$ Topologically Ordered Phases and Triple Linking Invariant of Loop Braiding, Phys. Rev. X 4, 031048 (2014).

[37] C. Wang, C.-H. Lin, and M. Levin, Bulk-Boundary Correspondence for Three-Dimensional Symmetry-Protected Topological Phases, Phys. Rev. X 6, 021015 (2016).

[38] Y. Wan, J. C. Wang, and H. He, Twisted gauge theory model of topological phases in three dimensions, Phys. Rev. B 92, 045101 (2015).

[39] A. Kapustin and R. Thorngren, Anomalies of discrete symmetries in various dimensions and group cohomology, arXiv:1404.3230.

[40] X. Chen, A. Tiwari, and S. Ryu, Bulk-boundary correspondence in (3+1)-dimensional topological phases, Phys. Rev. B 94, 045113 (2016).

[41] J. Wang, X.-G. Wen, and S.-T. Yau, Quantum statistics and spacetime surgery, Phys. Lett. B 807, 135516 (2020).

[42] A. Tiwari, X. Chen, and S. Ryu, Wilson operator algebras and ground states of coupled BF theories, Phys. Rev. B 95, 245124 (2017).

[43] Y. Peng, Gauge theory of strongly-correlated symmetric topological phases, Acta Phys. Sin. 69, 077102 (2020).

[44] B. Han, H. Wang, and P. Ye, Generalized Wen-Zee terms, Phys. Rev. B 99, 205120 (2019).

[45] AtMa P. O. Chan, P. Ye, and S. Ryu, Braiding with Borromean Rings in $(3+1)$-Dimensional Spacetime, Phys. Rev. Lett. 121, 061601 (2018).

[46] E. Witten, Quantum field theory and the Jones polynomial, Commun. Math. Phys. 121, 351 (1989).

[47] Gary T Horowitz, Exactly soluble diffeomorphism invariant theories, Commun. Math. Phys. 125, 417 (1989).
[48] J. C. Baez and J. Huerta, An invitation to higher gauge theory, Gen. Relativ. Gravit. 43, 2335 (2011).

[49] P. Ye and J. Wang, Symmetry-protected topological phases with charge and spin symmetries: Response theory and dynamical gauge theory in two and three dimensions, Phys. Rev. B 88, 235109 (2013).

[50] P. Ye and Z.-C. Gu, Vortex-Line Condensation in Three Dimensions: A Physical Mechanism for Bosonic Topological Insulators, Phys. Rev. X 5, 021029 (2015).

[51] A. Vishwanath and T. Senthil, Physics of Three-Dimensional Bosonic Topological Insulators: Surface-Deconfined Criticality and Quantized Magnetoelectric Effect, Phys. Rev. X 3, 011016 (2013).

[52] P. Ye, M. Cheng, and E. Fradkin, Fractional $s$-duality, classification of fractional topological insulators, and surface topological order, Phys. Rev. B 96, 085125 (2017).

[53] P. Ye, T. L. Hughes, J. Maciejko, and E. Fradkin, Composite particle theory of three-dimensional gapped fermionic phases: Fractional topological insulators and charge-loop excitation symmetry, Phys. Rev. B 94, 115104 (2016).

[54] J. Milnor, Link groups, Ann. Math. 59, 177 (1954).

[55] B. Mellor and P. Melvin, A geometric interpretation of Milnor's triple linking numbers, Algebr. Geom. Topol. 3, 557 (2003).

[56] M. A. Levin and X.-G. Wen, String-net condensation: A physical mechanism for topological phases, Phys. Rev. B 71, 045110 (2005).

[57] R. Dijkgraaf and E. Witten, Topological gauge theories and group cohomology, Commun. Math. Phys. 129, 393 (1990).

[58] T. Iadecola, T. Neupert, C. Chamon, and C. Mudry, Wire constructions of Abelian topological phases in three or more dimensions, Phys. Rev. B 93, 195136 (2016).

[59] T. Iadecola, T. Neupert, C. Chamon, and C. Mudry, Groundstate degeneracy of non-Abelian topological phases from coupled wires, Phys. Rev. B 99, 245138 (2019).

[60] Z.-C. Gu, J. C. Wang, and X.-G. Wen, Multikink topological terms and charge-binding domain-wall condensation induced symmetry-protected topological states: Beyond ChernSimons/BF field theories, Phys. Rev. B 93, 115136 (2016).

[61] Z.-F. Zhang and P. Ye (unpublished).

[62] D. Gaiotto, A. Kapustin, N. Seiberg, and B. Willett, Generalized global symmetries, J. High Energy Phys. 02 (2015) 172.

[63] A. Kapustin and N. Seiberg, Coupling a QFT to a TQFT and duality, J. High Energy Phys. 04 (2014) 001.

[64] C. W. von Keyserlingk and F. J. Burnell, Walker-wang models and axion electrodynamics, Phys. Rev. B 91, 045134 (2015).

[65] K. Walker and Z. Wang, (3+1)-TQFTs and topological insulators, Front. Phys. 7, 150 (2012). 